Journal of Patient-Centered

\title{
Abstracts From the 23rd Annual Health Care Systems Research Network Conference, March 21-23, 2017, San Diego, California
}

Follow this and additional works at: https://aah.org/jpcrr

Part of the Cardiology Commons, Family Medicine Commons, Geriatrics Commons, Health and Medical Administration Commons, Health Information Technology Commons, Oncology Commons, Pediatrics Commons, Preventive Medicine Commons, and the Public Health Commons

\section{Recommended Citation}

Abstracts from the 23rd annual Health Care Systems Research Network Conference, March 21-23, 2017, San Diego, California. J Patient Cent Res Rev. 2017;4:144-202. doi: 10.17294/2330-0698.1583

Published quarterly by Midwest-based health system Advocate Aurora Health and indexed in PubMed Central, the Journal of Patient-Centered Research and Reviews (JPCRR) is an open access, peer-reviewed medical journal focused on disseminating scholarly works devoted to improving patient-centered care practices, health outcomes, and the patient experience. 


\title{
Abstracts From the $23^{\text {rd }}$ Annual Health Care Systems Research Network Conference, March 21-23, 2017, San Diego, California
}

\author{
Advancing Learning Health Systems Through Embedded Research
}

\section{AGING AND MULTIMORBIDITY}

P1.01

Multimorbidity Profile of Urologic Patients in a
Large, Integrated Health Care Delivery System

Tullika Garg, ${ }^{1}$ Amanda Young, ${ }^{1}$ Korey Kost, ${ }^{1}$ H. Lester Kirchner $^{1}$

${ }^{1}$ Geisinger Health System, Danville, PA

Background: Two-thirds of urologic surgeries are performed in patients over 65 years old. As the American population ages, urologists are faced with increasingly complex older adults with multimorbidity. Our objective was to describe the multimorbidity profile of urologic patients in a large, integrated health care delivery system. We hypothesized that urologic patients are older and have more chronic conditions than the general primary care population.

Methods: We identified all Geisinger Health System (GHS) primary care patients from 2001 to 2015 and the subset that had at least one outpatient encounter in the urology department. The Agency for Healthcare Research and Quality's Clinical Classifications Software tool was applied to identify prevalent conditions based on diagnosis codes attached to outpatient visits, laboratory and pharmacy orders, and procedures.

Results: We identified 390,271 GHS primary care patients; 33,085 had at least one urology outpatient visit (8.5\%). Compared to the general GHS population, urology patients tended to be older (mean age: 48 years vs 61 years). Urology patients had a mean of 7 chronic conditions. The 5 most common conditions were hypertension, hyperlipidemia, prostate disorders, gastroesophageal reflux disease and other. The poster will include comparisons of urology population condition profiles to the GHS primary care population. We also will include chronic condition profiles by urologic condition.

Conclusion: Urologic patients were older compared to the general GHS primary care population and had significant numbers of chronic conditions. Multimorbidity profiles in the urologic population may be used to inform future efforts toward surgical prognostication and decision-making.
P1.02

Outcomes of an Embedded Resource and Education Program for Patients and Families With Memory Loss

Leah Hanson, ${ }^{1}$ Anna Forsberg, ${ }^{1}$ Avis Thomas, ${ }^{1}$ Jean Crow, ${ }^{1}$ Heidi Haley-Franklin, ${ }^{2}$ Terry Barclay ${ }^{3}$

${ }^{1}$ HealthPartners Institute, St. Paul, MN; ${ }^{2}$ Alzheimer's Association Minnesota-North Dakota, Minneapolis, MN; ${ }^{3}$ HealthPartners Center for Memory \& Aging, St. Paul, MN

Background: Patients with a new diagnosis of memory loss or dementia and their families typically wait 2 years before connecting to and utilizing community resources. Lack of education and support frequently results in crisisdriven care and hospitalization for patients, as well as carerelated strain, depression and other negative outcomes for caregivers. Memory PREP (Patient Resource \& Education Program) is a 4-month embedded program for patients with dementia and their families that involves meeting with a social worker in person or by phone to cover a curriculum of disease education, support and connection to community resources.

Methods: Patients and their care partners (dyads, $\mathrm{n}=90$ ) were recruited from two sources: physician referral from HealthPartners Center for Memory \& Aging, and through a mailing to patients throughout our care delivery system who were identified to have a new diagnosis code of dementia in their electronic health record. The intervention consisted of 5 encounters with a masters-level, specialty-trained social worker and randomization to either phone-only or 2 in-person plus 3 phone visits. The intervention curriculum was similar and ad hoc phone support was available to both groups. Outcomes included disease knowledge, mood, social support, health, stress, caregiver burden and quality of life and were evaluated at baseline, after program completion (4 months) and at 8 months. Changes from baseline were assessed using paired t-tests; ANOVA was used for comparisons.

Results: The Memory PREP program significantly increased care partner's knowledge of Alzheimer's disease, emotional support and completion of care planning (eg, power of attorney, driving and safety plans, and use of support groups). The program was equally effective when administered by phone as it was when administered inperson. The program received strong positive feedback from 
participants, especially care partners and family members; $93 \%$ of participants were likely to recommend the program to others.

Conclusion: Health care providers are struggling to meet the needs of patients and families facing dementia. Education and support are an important part of the care plan that is often outsourced to community partners. This embedded program led to increased knowledge, feelings of support, and completion of important safety and planning actions. Delivery of this program by phone may be a useful option to reduce barriers of access.

\section{P1.03}

Proportions of Patient-Centered Outcomes and Utilization Attributable to Chronic Conditions Among Adults With Asthma and/or Chronic Obstructive Pulmonary Disease

\section{Terrence Murphy ${ }^{1}$ \\ ${ }^{1}$ Yale School of Medicine, New Haven, CT}

Background: Patients with chronic obstructive pulmonary disease (COPD) often have multiple chronic conditions. It has not been previously investigated what proportions of patient-centered outcomes and health care utilization can be attributed to individual chronic conditions that concurrently manifest among patients with asthma, COPD, or both.

Methods: Using data from rounds 1-3 from the cohorts initiated in years 2008-2012 of the Medical Expenditure Panel Survey, we evaluated all patients 40 years and older who had been told by a doctor that they currently had asthma, chronic bronchitis or emphysema. The total sample size was 3,486 patients, and data recorded at round 1 (baseline) were used as explanatory variables for occurrence of each of the seven dichotomous outcomes over a 1-year period corresponding to the time between rounds 1 and 3. Based on multivariable logistic regression, the average attributable fractions (AAFs) for 12 distinct chronic conditions were calculated for three patient-centered outcomes and four health care utilization outcomes. The 12 conditions were: angina, arthritis, asthma, cancer, congestive heart failure, cognitive decline, COPD, diabetes, high blood pressure, lung cancer, myocardial infarction, and stroke. The three patient-centered outcomes were: 7 or more disability days, incident mobility, and incident worsening in perceived health. The four utilization outcomes were: any emergency room (ER) visit, any hospitalization, any outpatient visit, and respiratory hospitalization.

Results: Outcome models — the models of the seven outcomes exhibited fair to good discrimination (C-statistics between $66 \%$ and $76 \%$ ) and calibration ranging from poor to excellent (Hosmer-Lemeshow P-values from 0.01 to 0.84$)$. Patient-centered outcomes - the 12 conditions collectively explained the great majority of each of these outcomes. Presented hereafter are the sums of the AAFs from all 12 conditions (followed in parentheses by the four conditions, in descending order, that yielded the largest AAFs of each outcome). The sum of AAFs for 7 or more disability days was $77.8 \%$ (arthritis $24.2 \%$, COPD $18.3 \%$, cognitive decline $13.9 \%$, asthma $8.4 \%$ ). Sum of AAFs for incident mobility was $72.1 \%$ (arthritis $29.5 \%$, COPD $10.4 \%$, asthma $10.4 \%$, high blood pressure $6.4 \%$ ). Sum of AAFs for incident worsening of perceived health was $68 \%$ (COPD 21.3\%, arthritis $15.4 \%$, high blood pressure $9.4 \%$, asthma $6.8 \%$ ). Utilization outcomes - the 12 conditions collectively explained somewhere between a small fraction through a minority of each utilization outcome. Sum of AAFs for any ER Visit was 17.7\% (COPD 4.4\%, high blood pressure $3.0 \%$, arthritis $2.6 \%$, cognitive decline $2.5 \%$ ). Sum of AAFs for any hospitalization was 15.6\% (COPD $4.3 \%$, arthritis $3.0 \%$, diabetes $2.1 \%$, high blood pressure $2.1 \%$ ). Sum of AAFs for any outpatient visit was 35\% (arthritis $12.8 \%$, high blood pressure $6.1 \%$, asthma $3.9 \%$, cancer 3.3\%). Sum of AAFs for respiratory hospitalization was $5 \%$ (COPD $4.0 \%$, diabetes $1.0 \%$ )

Conclusion: Despite largely similar performance of the underlying multivariable logistic models, the 12 chronic conditions explained a much greater share of the overall occurrence of the patient-centered outcomes than for the utilization outcomes. From among the 12 conditions, arthritis, COPD and asthma consistently account for substantive proportions of outcome events in the patientcentered domain.

\section{$\mathrm{P} 1.04$}

Age and Sex Differences in the Magnitude of, and Hospital Death Rates Associated With, Multiple Chronic Conditions in Older Adults Hospitalized With Acute Myocardial Infarction

\section{Mayra Tisminetzky, ${ }^{1}$ Jerry Gurwitz,, ${ }^{1}$ Hoa Nguyen, ${ }^{2}$ Robert Goldberg ${ }^{3}$ \\ ${ }^{1}$ Meyers Primary Care Institute, Worcester, $M A ;{ }^{2}$ Baylor Scott \& White Health, Dallas, TX; ${ }^{3}$ University of Massachusetts Medical School, Worcester, MA}

Background: Despite the high prevalence of accompanying multimorbidity in older patients hospitalized with acute myocardial infarction, there are limited data available describing the burden of multimorbidity in different age strata of elderly men and women. The objectives of this study were to examine age- and sex-specific differences in the frequency and impact of multimorbidity on shortterm outcomes in older persons hospitalized with acute myocardial infarction.

Methods: The study population consisted of 3,973 patients hospitalized with acute myocardial infarction on a biennial basis at the 11 medical centers in Worcester, Massachusetts, between 2001 and 2011 . 
Results: The mean age of this population was 78 years and almost half were men. In the younger older group (65-74 years), 1 in 5 women versus 1 in 4 men presented 5 or more of the 11 chronic conditions examined. The proportion of younger older women receiving evidence-based in-hospital medications and cardiac interventions was significantly lower compared to men in the same age group. Among older old patients ( $>75$ years), men were more likely to be diagnosed with NSTEMI and presented with a higher prevalence of chronic conditions compared to similarly aged women. Similar proportions of men and women in the older old group received evidence-based in-hospital medications. A higher prevalence of multimorbidity was significantly associated with a greater risk of dying during the index hospitalization among younger older patients, but not in the older old groups.

Conclusion: The prevalence of multimorbidity in persons hospitalized with an acute myocardial infarction is significant and highly associated with the risk of dying in younger old men and women. Our findings highlight the importance of screening for these chronic conditions in older patients hospitalized with acute myocardial infarction.

\section{P1.05}

Research Priorities to Advance the Science of Multiple Chronic Conditions in Older Adults From the AGING Initiative Steering and Advisory Committees

Mayra Tisminetzky, ${ }^{1}$ Kathryn Anzuoni, ${ }^{1}$ Ben Shirley, ${ }^{2}$ Jay Magaziner, ${ }^{3}$ Elizabeth Bayliss, ${ }^{4}$ Jerry Gurwitz ${ }^{1}$

${ }^{1}$ Meyers Primary Care Institute, Worcester, MA; ${ }^{2}$ University of North Carolina, Chapel Hill, NC; ${ }^{3}$ University of Maryland School of Medicine, Baltimore, MD; ${ }^{4}$ Kaiser Permanente Colorado, Denver, CO

Background: There is an increasing recognition of the substantial gaps in knowledge that exist surrounding the health and health care of older adults with multiple chronic conditions (MCCs). A survey was developed to rank and prioritize research topics relevant to advancing the science of multimorbidity in the geriatric population.

Methods: A web-based survey was developed containing 37 topics organized into 11 domains. Topics were gathered from white papers, grant announcements or funded research projects relating to older adults that included the terms MCCs, multimorbidity and/or comorbidity. The search included the years 2010-2015 and employed the PubMed database as well as websites of the Agency for Healthcare Research and Quality, Patient-Centered Outcomes Research Institute (PCORI), National Institutes of Health, National Academy of Medicine and Google Scholar.

Results: Of the 366 respondents to the survey, $37 \%$ were investigators affiliated with the Health Care Systems Research Network, 25\% were affiliated with the Claude D.
Pepper Older Americans Independence Centers, 22\% were affiliated with academic health centers, $5 \%$ were affiliated with the Veterans Affairs system, 5\% were affiliated with federal agencies, $1 \%$ were affiliated with PCORI, and $4 \%$ were affiliated with other organizations. Based on toptwo box methodology, the top 10 research topics were: interactions between medications, disease processes and health outcomes in older adults with MCCs; health-related quality of life in older adults with MCCs; assessment tools in older adults with MCCs (to assess symptom burden, quality of life, function, etc); shared decision-making to enhance care planning in older adults with MCCs; disability in older adults with MCCs; symptom burden in older adults with MCCs; tools to improve clinical decision-making in older adults with MCCs; the role of the caregiver in caring for older adults with MCCs; self-management interventions in older adults with MCCs; and management of pain or other symptoms in older adults with MCCs.

Conclusion: Our findings are complementary to prior efforts to create a coherent and comprehensive research agenda to address the complex challenges facing these "high-need, high-cost" populations of older adults and the health care systems struggling to serve them. Future efforts should incorporate the views of patients, caregivers and other stakeholders to validate and broaden this important research agenda in this growing population.

\section{P1.06 \\ Obesity, Falls, and Hip Fractures Among Nursing Home Residents}

\section{Ning Zhang ${ }^{1}$}

\section{${ }^{1}$ Meyers Primary Care Institute, Worcester, MA}

Background: Aim was to examine the association between obesity status and the occurrence of falls and hip fracture among newly admitted nursing home residents.

Methods: This was a cohort study of newly admitted nursing home residents using national data from 2006 to 2010. Nursing homes measurements: Using the U.S. Minimum Data Set, we determined the occurrence of falls and hip fracture among newly admitted nursing home residents, according to obesity status, based on information available from the first quarterly assessment. Residents were categorized by body mass index as normal-to-overweight $(18.5$ to $<30)$, mildly obese $(30$ to $<35)$ and severely obese $(\geq 35)$.

Results: Among newly admitted nursing home residents, $55.1 \%$ of nonobese residents, $50.4 \%$ of mildly obese residents and $44.0 \%$ of severely obese residents experienced a fall. We also found that $6.9 \%$ of nonobese residents, $4.4 \%$ of mildly obese residents and $2.9 \%$ of severely obese residents experienced a hip fracture. After adjustment for resident-level and facility-level characteristics, mildly obese residents were $8 \%$ less likely (odds ratio [OR]: $0.92 ; 95 \%$ 
confidence interval [CI]: 0.91-0.93) and severely obese residents $16 \%$ less likely (OR: $0.84 ; 95 \%$ CI: $0.83-0.85$ ) to experience a fall compared with nonobese residents. Mildly obese residents were $20 \%$ less likely (OR: $0.80 ; 95 \%$ CI: $0.78-0.83$ ) and severely obese residents $29 \%$ less likely (OR: $0.71 ; 95 \%$ CI $0.68-0.74$ ) to experience a hip fracture compared with nonobese residents.

Conclusion: Obesity is associated with reduced risk for falls and hip fracture among nursing home residents. Future studies are needed to examine the reasons for the associations noted and to better understand the implications for care of obese nursing home residents.

\section{CANCER}

P2.01

Lung Cancer Screening: A Qualitative Study Exploring the Decision to Opt Out of Screening

\section{Lisa Carter-Harris, ${ }^{1}$ Susan Brandzel, ${ }^{2}$ Karen Wernli, ${ }^{2}$ Joshua Roth, ${ }^{3}$ Diana Buist ${ }^{2}$}

${ }^{1}$ Indiana University Purdue University at Indianapolis, Indianapolis, IN; ${ }^{2}$ Group Health Research Institute, Seattle, WA, ${ }^{3}$ Fred Hutchinson Cancer Center, Seattle, WA

Background: Lung cancer screening with annual low-dose computed tomography is relatively new for long-term smokers in the United States, and supported by a U.S. Preventive Services Task Force Grade B recommendation. As lung cancer screening programs are more widely implemented and providers engage patients about screening, it is critical to understand what influences a patient's decision to screen, or not, for lung cancer. Understanding lung cancer screening behavior among high-risk smokers who opt out provides insight from the patient perspective about the shared decision-making process for lung cancer screening. The purpose was to explore screening-eligible patients' decision to opt out of lung cancer screening after receiving a provider recommendation. This knowledge will inform intervention development to enhance shared decision-making processes between long-term smokers and their providers and decrease decisional conflict about lung cancer screening.

Methods: Semi-structured qualitative telephone interviews were performed with 18 lung screening-eligible men and women who were members of an integrated, mixed-model health care system in Seattle, Washington, about their decision to opt out of lung cancer screening. Participants met lung cancer screening criteria for age, smoking and packyear history. Audio-recorded interviews were transcribed verbatim. Two researchers with cancer screening and qualitative methodology expertise conducted data analysis using thematic content analytic procedures.

Results: Participant mean age was 66 years (standard deviation: 6.5). Majority were female (61\%), Caucasian (83\%) or current smokers (61\%). Five themes emerged: 1) Knowledge Avoidance; 2) Perceived Low Value; 3) False-Positive Worry; 4) Practical Barriers; and 5) Patient Misunderstanding.

Conclusion: Many screening-eligible smokers opt out of lung cancer screening. The participants in our study provided new insights into why some patients make this choice. While there are known drawbacks to lung cancer screening, and it is not necessarily the best option for everyone who is eligible, it is known to be effective in early lung cancer detection among high-risk patients. Understanding why people decide not to screen will enhance future efforts to improve knowledge transfer from providers to patients about the risks and benefits of lung cancer screening and ultimately enhance shared decision-making about lung cancer screening.

\section{P2.02}

\section{Incidental Thyroid Nodules: A Radiologic Epidemic}

\section{Steven Chang ${ }^{1}$}

\section{${ }^{1}$ Henry Ford Health System, Detroit, MI}

Background: The incidence of well-differentiated thyroid cancer has risen dramatically over the last several decades. One proposed explanation for this is the rapid growth in utilization of different radiology studies, which have resulted in large numbers of incidentally identified thyroid nodules. Objectives were to: 1) determine, at our institution, the incidence of incidentally identified thyroid nodules requiring fine needle aspiration; 2) describe the imaging modality and indication for these incidentally identified nodules; and 3) assess the outcomes, including surgical rates, among these nodules.

Methods: A retrospective review was performed of all patients who underwent fine needle aspiration(s) of thyroid nodules between January 2006 and December 2010 by the endocrinology division at a large academic medical center. Medical records were reviewed to identify whether the biopsied thyroid nodule was discovered incidentally through nonthyroid-related imaging or whether it was identified by palpation by patient or physician. Demographic, radiological, surgical and pathological data were assessed.

Results: Fine needle aspirations were performed on 2,296 total thyroid nodules and 1,794 patients; $24 \%(n=431)$ of patients underwent a biopsy for incidentally identified nodules. The most common indications documented for the initial imaging that resulted in an incidental finding of a nodule were neck pain (32.4\%), nonthyroid cancer workup $(26 \%)$ and evaluation for pulmonary embolus (12.8\%). Chest computed tomography (CT), magnetic resonance imaging of the spine or neck, and CT of the neck were the most common imaging modalities that led to thyroid incidentalomas $(29.2 \%, 19.9 \%$ and $16.1 \%$, respectively). 
Rates of surgery and identification of cancer did not differ significantly based on the modality or indication for imaging. Conclusion: In this study, nearly a quarter of patients undergoing fine needle aspiration had their thyroid nodule identified incidentally on imaging. With the continued proliferation of radiology studies, this flood of thyroid nodule incidentalomas is likely to continue to expand.

\section{P2.03}

\section{Diabetes Treatment and Risks of Adverse Breast Cancer Outcomes Among Elderly Breast Cancer Patients: A SEER-Medicare Analysis}

\author{
Lu Chen, ${ }^{1}$ Jessica Chubak, ${ }^{1}$ Denise Boudreau, ${ }^{1}$ William \\ Barlow, ${ }^{2}$ Noel Weiss, ${ }^{3}$ Christopher $\mathrm{Li}^{4}$
}

${ }^{1}$ Group Health Research Institute, Seattle, WA; ${ }^{2}$ Cancer Research and Biostatistics, Seattle, WA; ${ }^{3}$ University of Washington, Seattle, WA; ${ }^{4}$ Fred Hutchinson Cancer Research Center, Seattle, WA

Background: Metformin, a first-line diabetes treatment, is hypothesized to lower the risk of incident breast cancer, but it is unclear whether metformin or other antidiabetic medications influence the likelihood of adverse breast cancer outcomes.

Methods: A retrospective cohort study was conducted using the linked Surveillance, Epidemiology and End-Results (SEER)-Medicare database. Diabetic and nondiabetic women were included if they were aged 66-80 years, newly diagnosed with stage I or II breast cancer, and enrolled in Medicare Parts A, B and D during 2007-2011. Information on filled diabetes-related prescription medications was obtained from Medicare Part D claims data. Our primary outcomes of interest were a second breast cancer event and breast cancer-specific mortality. Time-varying Cox proportional hazard models were used to estimate hazard ratios (HR) and their associated 95\% confidence intervals (CI).

Results: Among 14,766 women included in the study, 791 were identified as having had a second breast cancer event, 627 had a recurrence and 237 died from breast cancer. Use of metformin after breast cancer $(n=2,558,17.3 \%)$ was associated with 28\% (HR: 0.72 , 95\% CI: 0.57-0.92), 31\% (HR: 0.69, 95\% CI: 0.53-0.90) and 49\% (HR: 0.51, 95\% CI: $0.33-0.78)$ lower risks of a second breast cancer event, breast cancer recurrence and breast cancer death, respectively, compared to metformin nonusers. Use of sulfonylureas $(\mathrm{n}=1,701,11.5 \%)$ was associated with 1.49 (95\% CI: 1.00-2.23) higher risk of breast cancer death. A 2.58-fold higher risk (95\% CI: 1.72-3.90) of breast cancer death also was seen among insulin users $(n=1,099$, $7.4 \%$ ). In assessing potential confounding by indication, similar patterns were observed in analyses restricted to pharmacologically treated diabetic patients.

Conclusion: We observed variation in the relationship between different diabetes medications and risk of adverse breast cancer outcomes, with metformin associated with reduced risks and sulfonylureas and insulin with increased risks. Pending confirmation of these results, metformin may be a preferred treatment for diabetes among breast cancer survivors, and further research examining its benefits among nondiabetic patients may be warranted.

P2.04

Reasons for Never and Intermittent Completion of Colorectal Cancer Screening After Receiving Multiple Rounds of Mailed Fecal Tests

Beverly Green, ${ }^{1}$ June BlueSpruce, ${ }^{1}$ Leah Tuzzio, ${ }^{1}$ Sally Vernon, ${ }^{2}$ L. Aubree Shay, ${ }^{2}$ Sheryl Catz ${ }^{3}$

${ }^{1}$ Group Health Research Institute, Seattle, WA; ${ }^{2}$ University of Texas School of Public Health, Houston, TX; ${ }^{3}$ Betty Irene Moore School of Nursing, University of California, Davis, Sacramento, $C A$

Background: Long-term adherence to colorectal cancer (CRC) screening is particularly important for fecal testing. As few as a quarter of patients repeat fecal testing the next year in some U.S. settings. The purpose of this qualitative study was to identify barriers and facilitators reported by patients with suboptimal screening adherence to refine interventions for ongoing adherence to CRC screening. We also explored whether participants, particularly never screeners, would be willing to do a CRC screening blood test.

Methods: 41 patients who previously enrolled in the Systems of Support to Increase CRC Screening (SOS) trial were interviewed 4-5 years later. Participants were purposively selected to include men and women with diverse race/ethnicities who had either been inconsistent screeners or had never screened during the first 3 years of SOS, despite receiving at least 2 rounds of mailed fecal tests. Two interviewers conducted an approximately 30-minute telephone interview using a semi-structured interview guide. An iterative thematic analysis approach was used.

Results: Barriers were more pervasive among neverscreeners: 1) Avoidance; 2) Aversion to stool; 3) Health concerns; and 4) Fear. Facilitators were more often mentioned by repeat-screeners: 1) The simpler 1-sample test; 2) Mailings and testing at home convenience; 3) Prevention; and 4) Social influence. Participants had diverse preferences for types (phone, mail) with some not preferring email links to the electronic health record. A nurse not from their clinic calling was acceptable if the nurse was knowledgeable about their records and could communicate with their physician. Participants, especially never-screeners, were enthusiastic about a screening blood test.

Conclusion: Future CRC screening programs should be designed to minimize these barriers and maximize facilitators to improve long-term screening adherence. 
P2.05

Systems of Support to Increase Colorectal Cancer (CRC) Screening - A Randomized Trial to Increase Long-Term Adherence to CRC Screening: Time in Compliance Over 5 Years

Beverly Green, ${ }^{1}$ Melissa Anderson, ${ }^{1}$ Jessica Chubak, ${ }^{1}$ Andrea Cook, ${ }^{1}$ Sharon Fuller, ${ }^{1}$ Richard Meenan, ${ }^{2}$ Sally Vernon ${ }^{3}$

${ }^{1}$ Group Health Research Institute, Seattle, WA; ${ }^{2}$ Kaiser Permanente Center for Health Research, Portland, OR; ${ }^{3}$ University of Texas School of Public Health, Houston, TX

Background: Colorectal cancer (CRC) is the secondleading cause of cancer deaths. Morbidity and mortality could be rapidly reduced through higher uptake and adherence to CRC screening. Information on longer-term screening adherence comes from organized programs that lack a comparison group. Systems of Support to Increase Colorectal Cancer Screening and Follow-Up (SOS) is an ongoing trial testing a centralized mailed and phone-based program to increase long-term CRC screening adherence. We hypothesized that, compared to usual care, interventionarm patients would have more time in compliance with CRC screening guidelines over 5 years.

Methods: Design: Individual randomized controlled trial. Setting: Integrated health care organization in Washington State. Usual care included patient-centered medical home and clinic-based interventions to increase CRC screening. Participants: 4,675 individuals initially aged $50-74$, without CRC or first-degree relative with CRC before age 60, no inflammatory bowel disease, no life-limiting disease, and not current for CRC screening. All participants contributed data but were censored at disenrollment, death, age 76 or diagnosis of CRC. Interventions: Included a mailed pamphlet on CRC screening choices, a call-in number if colonoscopy was preferred and mailed fecal tests for those not choosing colonoscopy. Patients were randomly assigned in years 1 and 2 to receive this only, this plus brief telephone assistance, or both mailings and assistance and nurse navigation for those still unscreened. In year 3, intervention group participants still CRC screening-eligible were randomized to stopped or continued mailed interventions in years 3 and 5. Primary outcome (a priori): The percent of time covered for CRC screening testing over 5 years (2008-2014). Screening tests contributed covered time based on national guidelines for screening intervals (fecal tests annually, sigmoidoscopy 5 years, colonoscopy 10 years).

Results: On average over 5 years, intervention participants had 224 more days of covered time, or $31 \%$ more time not in need of CRC testing (rate ratio, weighted for exposure time and adjusted relative risk $1.31(1.25,1.37)$. Fecal testing was responsible for almost all additional covered time.

Conclusion: An organized mail and phone program led to increased CRC screening adherence over 5 years, mainly because of regular fecal testing uptake.
P2.06

\section{A Cost-Effectiveness Analysis of an Adenocarcinoma Risk Prediction Biomarker Test for Patients With Barrett's Esophagus}

\author{
Jing Hao, ${ }^{1}$ Susan R. Snyder, ${ }^{1}$ Rebecca Critchley- \\ Thorne $^{2}$ \\ ${ }^{1}$ Geisinger Health System, Danville, PA; ${ }^{2}$ Cernostics Inc., \\ Pittsburgh, PA
}

Background: Current surveillance guidelines for identifying patients with Barrett's esophagus (BE) at high risk of progressing to esophageal adenocarcinoma (EAC) are considered inefficient because less than $1 \%$ of all $\mathrm{BE}$ patients are likely to develop EAC. A new tissue systems pathology risk prediction test (RPT) stratifies BE patients by their risk of progression to high-grade dysplasia or EAC by assigning them to low-, intermediate- or high-risk categories based on an individualized risk score. These categories can be used to guide endoscopic surveillance and treatment decisions. This study evaluates the cost-effectiveness of this new RPT versus the current standard of care for surveillance and treatment of BE.

Methods: Decision analysis with Markov modeling and simulation were used to compare cost and quality-adjusted life-years (QALYs) from the perspective of a U.S. health insurer with care delivered by an integrated health system. Model assumptions and disease progression probabilities were derived from the literature. Performance metrics for the RPT were from an independent clinical validation study. Cost of the RPT was based on reimbursement rates from multiple payers. Other costs were derived from Geisinger Health System payment data.

Results: Base-case results of a 5-year model indicated an incremental cost-effectiveness ratio (ICER) of the RPT compared to the standard of care of \$50,181/QALY in 2012 U.S. dollars. RPT versus standard of care total surveillance endoscopies over the 5-year period were $17.6 \%$ lower, and endoscopic treatments (radiofrequency ablation and/ or endoscopic mucosal resection) were $64.7 \%$ higher. The RPT also reduced the number of cases of high-grade dysplasia, EAC and EAC-related deaths by $55.1 \%$, 49.6\% and $37.1 \%$, respectively. Sensitivity analysis indicated that the probability of the RPT being cost-effective compared to the standard of care was $56.4 \%$ at the $\$ 100,000$ /QALY acceptability threshold. The following changes in the model variables can make the RPT more cost-effective: higher BE-to-EAC progression rate, lower RPT cost, higher endoscopy cost, lower EAC utility, and higher physician RPT adherence/use.

Conclusion: The new RPT is cost-effective after 5 years and improves patient outcomes due to improving the effectiveness of surveillance and treatment protocols, resulting in fewer patients transitioning to high-grade dysplasia, EAC and death. 
P2.07

Can Thyroid Cancer Overdiagnosis, Overtreatment, and Costs Be Reduced by Following Guidelines?

\author{
Dina Hassen, ${ }^{1}$ Susan R. Snyder, ${ }^{1}$ Steven Chang, ${ }^{2}$ Jing \\ Hao, ${ }^{1}$ Michael Singer, ${ }^{2}$ Arti Bhan ${ }^{2}$ \\ ${ }^{1}$ Geisinger Health System, Danville, PA; ${ }^{2}$ Henry Ford Health \\ System, Detroit, MI
}

Background: There is worldwide consensus on an alarming increase in overdiagnosis and overtreatment of papillary thyroid cancer in recent decades, mostly attributable to increased screening with more advanced diagnostic technology (eg, ultrasound, computed tomography and magnetic resonance imaging) detecting small papillary nodules. In 2009, evidence-based guidelines by the American Thyroid Association recommended against routine fine needle aspiration for unsuspicious papillary nodules of $<1$ $\mathrm{cm}$. The purpose of this study is to compare the change in the ratio of patients with thyroid cancer to all patients with thyroid nodules before and after the issuance of the 2009 guidelines as well as to provide national health care cost estimates.

Methods: The hypothesis is adherence to evidence-based guidelines targeting inappropriate testing of small nodules, which are highly prevalent in the general population and are mostly benign $(\sim 80 \%)$, will be effective at reducing overdiagnosis of thyroid cancer without significant harm to patients with malignant nodules $(\sim 20 \%)$, which can be tested and treated when they are larger. We use the Medical Expenditure Panel Survey (MEPS), which provides annual nationally representative cross-sectional data on respondents' health service utilization as well as the cost of these services. Binary variables will be used to denote the presence of thyroid nodules and thyroid cancer in adults based on three-digit ICD-9-CM diagnosis condition codes.

Results: MEPS data estimates will be used to compare the difference in the proportion of respondents with thyroid cancer to thyroid nodules before and after the issuance of the 2009 guidelines and measure the cost of thyroid nodules over the recent 10-year period, 2005-2014, by using generalized linear models with a log-link function on total, inpatient, outpatient and prescription medication health care expenditures.

Conclusion: The results of this analysis will contribute additional information to the growing body of evidence on overdiagnosis and overtreatment as well as the financial burden of thyroid cancer in the United States. It highlights the need for further study using detailed medical record data inclusive of nodule size and diagnostic procedures to determine the extent of guideline adherence and whether better adherence would effectively address a substantial portion of the overdiagnosis and overtreatment problem.
P2.08

\section{External Validity of Electronic Health Record Studies of Cancer Patients}

Anqi Jin, ${ }^{1}$ Scarlett Gomez, ${ }^{2}$ Harold Luft, ${ }^{1}$ Daphne Lichtensztajn, ${ }^{2}$ Caroline Thompson ${ }^{3}$

${ }^{1}$ Palo Alto Medical Foundation Research Institute, Palo Alto, CA; ${ }^{2}$ Cancer Prevention Institute of California, Fremont, CA; ${ }^{3}$ San Diego State University, San Diego, CA

Background: Electronic health records (EHRs) from academic and community-based health care systems are increasingly used for epidemiologic and health services research. The external validity of study findings is often unreported, and some question the representativeness of the patient population. We evaluate the generalizability of Sutter Health cancer patients and potential bias in cancer research based on available EHR data.

Methods: We linked the patient population of Sutter Health, a large, multispecialty health care delivery system in Northern California, with the statewide, populationbased California Cancer Registry and compared the distributions of demographic, socioeconomic and cancer characteristics for two groups: 1) all Sutter Health members diagnosed with cancer in 2012-2013, and 2) all cancer patients in the 17-county Sutter Health catchment region for the same period. To evaluate potential bias of EHR data, a validation study was conducted to additionally compare those characteristics among Sutter patients who had cancer-related charges or encounters in the Sutter EHR system with the catchment region, also for 2012 and 2013.

Results: $43.1 \%(\mathrm{~N}=69,344)$ of cancer patients diagnosed from 2012 to 2013 in the catchment region were Sutter patients. Compared with all regional cancer patients, Sutter's population had proportionally more non-Hispanic whites $(70.5 \%$ vs $65.2 \%)$, had slightly more breast cancer patients $(32.9 \%$ vs $29.6 \%)$ and were more likely to have Medicare $(37.2 \%$ vs $28.0 \%$ ), but they were similar in terms of age, gender, socioeconomic status, tumor stage and treatment types. Our validation study showed that $28.5 \%$ of 69,344 cancer patients diagnosed during 2012-2013 in the catchment area were Sutter patients with EHR information available. These Sutter patients with EHR information have more comparable distributions to the underlying cancer patient population, with the exceptions of payer source, for which they were more likely to pay with Medicare ( $38.8 \%$ vs $28.8 \%$ ), and race/ethnicity distribution, as they represented more non-Hispanic whites (71.1\% vs $65.2 \%)$.

Conclusion: Research based on EHRs from single or integrated health care systems have unknown generalizability. We found that cancer patients from Sutter Health are generally representative of the underlying population, thus, cancer research based on Sutter EHR data can provide good external validity, while minimizing potential biases. 
P2.09

\section{Establishing a Population-Based Cohort of Nonmuscle Invasive Bladder Cancer Cases to Improve Care by Enhancing Surveillance and Risk Stratification}

\author{
Carmit McMullen, ${ }^{1}$ Maureen O'Keeffe-Rosetti, ${ }^{1}$ Sheila \\ Weinmann, ${ }^{1}$ Michael Leo, ${ }^{1}$ Matthew Nielsen ${ }^{2}$
}

${ }^{1}$ Kaiser Permanente Center for Health Research, Portland, OR; ${ }^{2}$ School of Medicine, University of North Carolina, Chapel Hill, NC

Background: Among the $\sim 75 \%$ of bladder cancer patients who present with superficial (stage: $<\mathrm{T} 2$ ) disease, there is substantial variability in the progression to muscle-invasive (stage: $\geq \mathrm{T} 2$ ) disease. An expert panel representing the American Urological Association has expressed the need for population-based data and better predictive tools for bladder cancer surveillance. However, an existing risk-calculator has faced limited adoption, possibly because of reliance on aggregate data from clinical trial populations and failure to design the risk-calculator for use in specific clinical contexts ("use cases").

Methods: We are developing a risk-assessment tool using a population-based cohort from Kaiser Permanente Northwest (KPNW). The cohort includes all superficial bladder cancer cases diagnosed and treated at KPNW from 1990 to 2014. Data from tumor registry, pathology and health plan membership files comprise our database. We conducted in-depth interviews with 8 urologists in a variety of practice settings to identify use cases for improving clinical care through personalized risk-assessment. Our analysis describes key characteristics of our cohort as well as stakeholder suggestions for how a risk-calculator could enhance care.

Results: We identified 2,131 cases of superficial bladder cancer. The population had a median of 2.0 pathology records per case, ranging up to 24 records, with $95 \%$ of cases having 6 or fewer records. Half of the population did not have a recurrence or progression of bladder cancer during follow-up. Overall, $86 \%$ of pathology reports per case reported positive bladder cancer findings. Stakeholder interviews with urologists identified numerous use cases for personalized risk calculations of recurrence and progression. These included: enhancing patient communication and documentation about recurrence/progression; providing guidance about when to discontinue or reduce surveillance for very low-risk patients; identifying and creating scheduling and call-back supports for patients who have high priority for follow-up cystoscopy; and contextualizing bladder cancer mortality risk in the context of competing comorbid conditions. Urologists in general practice reported different use preferences than urologic oncologists.

Conclusion: By interviewing a variety of clinicians, we have identified a range of clinical decision support and patient education uses for a personalized risk-calculator for bladder cancer surveillance based on a large populationbased cohort reflecting "real world" practice.

P2.10

Patterns of Repeat Colorectal Cancer Screening and Follow-Up of Abnormal Results in an Integrated Safety-Net Health Care System

Caitlin Murphy, ${ }^{1}$ Amit Singal, ${ }^{1}$ Joanne Sanders, ${ }^{1}$ Sandi Pruitt, ${ }^{1}$ Simon Craddock Lee, ${ }^{1}$ Ethan Halm, ${ }^{1}$ Celette Sugg Skinner ${ }^{1}$

${ }^{1}$ University of Texas Southwestern Medical Center, Dallas, TX

Background: Colorectal cancer (CRC) screening with stoolbased tests, including fecal immunochemical test (FIT), can reduce CRC mortality if patients with normal test results repeat testing annually and those with abnormal results receive timely follow-up with colonoscopy. However, most studies focus on one discrete step rather than longitudinal adherence to screening (ie, undergoing repeat screening and timely follow-up). We examined patterns of repeat CRC screening and follow-up of abnormal results over a 4-year period at Parkland Health \& Hospital System, a large, integrated safety-net health care system in Dallas County, Texas.

Methods: Eligible patients were aged $50-60$ years, completed an index FIT between January 1, 2010, and December 31, 2010, and were followed through the end of the study period in 2014. Patients with a history of CRC, colectomy, or prior CRC screening with colonoscopy or sigmoidoscopy were excluded. We defined longitudinal adherence as a composite outcome of: 1) FIT screening with normal results in each calendar year; 2) crossover to endoscopy screening after one or more normal FITs; or 3) colonoscopy within 180 days following abnormal FIT. We also characterized inconsistent screening as being up-to-date with screening in none, one, two or three out of four possible calendar years.

Results: A total of 4,826 patients were included in the analysis. Most were Hispanic $(41.1 \%)$ or non-Hispanic black (38.1\%), female (65.1\%), and uninsured or receiving medical assistance/charity insurance $(80.2 \%)$. Across the study period, $834(17.3 \%)$ showed longitudinal adherence by completing repeat on-schedule screening with some combination of FIT and endoscopy. Some patients ( $\mathrm{n}=940$, $19.5 \%$ ) completed endoscopy after one or more normal FITs, and $367(7.6 \%)$ had an abnormal FIT result at some point during the study period. A decreasing proportion of patients were up-to-date with screening recommendations in one $(\mathrm{n}=1,740,36.1 \%)$, two $(\mathrm{n}=1,221,25.3 \%)$ or three $(\mathrm{n}=973,20.2 \%)$ out of four calendar years.

Conclusion: Less than $20 \%$ of patients in a safety-net health care system show longitudinal adherence to the CRC screening process, which may reduce the effectiveness of screening. Efforts to improve timely receipt of repeat FIT 
and follow-up colonoscopy after abnormal FIT may increase the proportion of minority and low-income patients up-todate with ongoing screening.

\section{P2.11}

Predictors of Colorectal Cancer Screening Prior to Implementation of a Large Pragmatic Trial in Federally Qualified Health Care Centers

Amanda Petrik, ${ }^{1}$ Thuy Le, ${ }^{2}$ Erin Keast, ${ }^{1}$ Jennifer Rivelli, ${ }^{1}$ Keshia Bigler, ${ }^{1}$ Beverly Green, ${ }^{3}$ William Vollmer, ${ }^{1}$ Gloria Coronado ${ }^{1}$

${ }^{1}$ Kaiser Permanente Northwest, Portland, OR; ${ }^{2}$ OCHIN, Portland, OR; ${ }^{3}$ Group Health Research Institute, Seattle, WA

Background: Colorectal cancer(CRC) screening can prevent unnecessary cancer deaths. Federally qualified health care centers $(\mathrm{FQHC})$ serve a unique patient population that often is not screened. Knowing who in the $\mathrm{FQHC}$ environment is getting screened via fecal testing (FIT/FOBT) and via colonoscopy can assist in tailoring intervention to raise rates of CRC screening.

Methods: As part of the STOP CRC study, we examined the associations among patient-level and neighborhood-level characteristics and being up to date with CRC screening guidelines (ie, having had a fecal test in the past year or having had a colonoscopy in the past 10 years). We also examined associations between these factors and being screened with a fecal test only. We derived neighborhoodlevel characteristics by linking U.S. Census data to zip code information obtained from the electronic health record. We used logistic regression, adjusted for clustering at the health center level, to calculate our associations.

Results: We observed a steady rise in CRC screening rates from 2010 to 2015 (17.7\% to 46.8\%). In adjusted analysis, factors associated with being up to date with CRC screening overall were: being older, having health insurance, having prior office or emergency room visits, and having other preventive screenings. Among patients who were up to date with CRC screening, factors associated with use of fecal testing were: being younger, speaking a non-English language, being uninsured, having prior office visits, having had a flu shot in past year, and living in a neighborhood with higher unemployment.

Conclusion: Encounter-level variables - such as insurance status, number of office visits in the past year, types of chronic health conditions, and other screenings - were most often associated with predicting CRC screening, and FIT/FOBT use was more common among typically underscreened populations. Our findings may inform clinicbased efforts to raise rates of CRC screening, especially in the community clinic setting.
P2.12

Adolescent and Young Adult Cancer Data in the Cancer Research Network

Rebecca Ziebell, ${ }^{1}$ Jessica Chubak $^{1}$

${ }^{1}$ Group Health Research Institute, Seattle, WA

Background: Cancer is the leading cause of diseaserelated death among U.S. persons aged 15-39 years. Certain cancers are more common in this adolescent/young adult (AYA) population than in younger children or older adults. Research supported by initiatives like the Cancer Research Network (CRN) is needed to address late effects of cancer and its treatments among AYA survivors. The CRN consortium is funded by the National Cancer Institute to support cancer research at a subset of Health Care Systems Research Network sites. The data collected and maintained by CRN sites facilitate a variety of AYA cancer research opportunities.

Methods: Counts and characteristics of primary malignant tumors diagnosed during 1996-2015 among health plan enrollees aged 15-39 were obtained from the Group Health virtual data warehouse. Descriptive analyses included counts of incident cases by selected demographic characteristics and cancer type. In early 2017, updated counts as well as postdiagnosis retention metrics will be obtained from Group Health and up to 11 additional CRN sites via a centrally developed Statistical Analysis System ${ }^{\circledR}$ program.

Results: During 1996-2015, 1,982 AYA Group Health enrollees (18\% adolescent, age $15-24 ; 82 \%$ young adult, age 25-39) were diagnosed with cancer. Females comprised $65 \%$ of total AYA diagnoses, although the disparity was less pronounced among adolescents (54\% female) than young adults $(67 \%)$. Whites accounted for $78 \%$ of all diagnoses, while Asians, blacks and Hispanics comprised 6.8\%, 3.6\% and $5.1 \%$ of diagnoses, respectively. Hodgkin lymphoma was the most commonly diagnosed cancer among adolescents (15\% of diagnoses), while breast cancer was the most commonly diagnosed (19\%) among young adults. Melanoma, thyroid cancer and testicular cancer were the second, third and fourth most commonly diagnosed malignancies among both adolescents and young adults.

Conclusion: The CRN supports the infrastructure to maintain high-quality data on AYA cancer patients at eight funded and four affiliate sites. Additionally, the multisite CRN environment allows for the study of AYA cancers among a large, demographically diverse patient population. Thus, the CRN provides a setting that is well suited for research in what is potentially one of the largest AYA cancer cohorts with longitudinal data. 


\section{CARE IMPROVEMENT}

\section{P3.01 \\ The Impact of Family Support on Health Care Utilization After Inpatient Care}

\section{Caroline Carlin, ${ }^{1}$ Guy David ${ }^{2}$ \\ ${ }^{1}$ Medica Research Institute, Minneapolis, $M N ;{ }^{2}$ University of Pennsylvania, Philadelphia, PA}

Background: Effective post-acute care following an inpatient stay is important to high-quality care, as it has been shown to prevent readmissions and complications. It has been argued that the presence of a family member serves as a substitute for formal care; however, there is very little research quantifying the impact of informal caregivers on patterns of post-acute health care utilization. We leverage health plan administrative data collected under managed Medicare coverages to explore the impact of informal caregivers on post-acute care and recovery.

Methods: Our population was Medicare-eligible members who had an inpatient admission in 2014-2015. Our "treatment" variable was the presence of another adult in the home; the outcome variables of interest include use of post-acute care (skilled nursing facility, home health care), intensity and reuse of inpatient care (length of stay, readmissions), emergency department visits after discharge and overall health care expenditures. In this pilot work, we use the term "spouse" to indicate another adult in the home to provide care to Medicare members postdischarge. We imputed the presence of a spouse by matching covered individuals by address (distinct to the apartment unit).

Results: We seek to determine how the presence of a spouse in the home, and the spouse's health status, affects transition from an inpatient setting. Our hypothesis is that the presence of a healthy spouse reduced the probability that post-acute care was needed and reduced the probability of emergency department visits and inpatient readmissions after discharge, resulting in lower overall expenditures. The presence of a spouse in poor health may actually increase the rate of post-acute care, relative to single members, because the need for transition care is less defined.

Conclusion: By understanding the impact of spousal support, we can better target transition care to improve patient outcomes. This pilot work provides preliminary data in support of survey funding to create better controls for family and friend support postdischarge.

\section{P3.02}

Choosing Reports Wisely: Considerations in Reporting for Clinical Improvement

Sharon Fuller, ${ }^{1}$ Matt Handley, ${ }^{1}$ Angela Sparks, ${ }^{1}$
Kimberly Wicklund ${ }^{1}$

${ }^{1}$ Group Health Research Institute, Seattle, WA
Background: Group Health has undertaken a number of clinical improvement initiatives arising from the ABIM Foundation Choosing Wisely ${ }^{\circledR}$ campaign. Currently, the particular focus is on reducing the use of antibiotics for upper respiratory infections. To support this effort, clear, timely, reliable reporting is required to understand the current state and motivate and recognize change.

Methods: Throughout the Choosing Wisely campaign, we have developed and refined our reporting methods, tools and dissemination strategies. We target reports to different audiences - numerical summaries for grant funding, clinic-level charts for clinical leaders, and detailed provider-level reporting. A single data set of visit-level data is used for all reports and rolled up to the appropriate level. We have focused much of our effort on providerlevel reporting. Goals include giving providers insights into their own practice patterns, highlighting improvements, and identifying peers who could be consulted for ideas on successfully changing practice. To this end, we have been working to develop reports that summarize rates, volume and temporal trends into a single easily interpreted graphic. Migrating to Tableau visualization software has been instrumental in this effort.

Results: For report consumers, we have developed reports that are regularly disseminated, clearly documented, visually appealing and easy to interpret. For report development, we have worked toward automation and adopted tools geared toward rapid data exploration. The rate of antibiotic prescription for upper respiratory infections has decreased sharply since reporting began. We have observed that providers appear engaged with the improvement process, and that leadership is enthusiastic about the potential for future developments in reporting to facilitate ongoing change. Long-term support from executive, clinical, information technology and research leadership has been critical to our work. We also benefit from provider feedback - we share visit-level data on request, enabling providers to do chart audits and report potential data quality problems.

Conclusion: With sustained work over time, we have developed a suite of reports that effectively support important clinical improvement efforts. We plan to adapt this reporting scheme for use with other quality measures as well. We also look forward to migrating to an online interactive platform where leaders and providers can access reports directly.

P3.03

Impact of Physician Practice Style on Costs, Clinical Quality, Patient Experience, Physician Productivity, and Physician Time

\author{
Su-Ying Liang, ${ }^{1}$ Laura Eaton, ${ }^{1}$ Sukyung Chung, ${ }^{1}$ Harold \\ Luft $^{1}$ \\ ${ }^{1}$ Palo Alto Medical Foundation Research Institute, Palo \\ Alto, $C A$
}


Background: During acute care visits, some primary care physicians (PCPs) typically focus on the presenting problem ("the focused"); others typically address additional issues ("max-packers"). Processes and outcomes may vary between these distinct practice styles. Max-packers, by managing additional conditions or preventive services during an acute care visit, may reduce the number of future visits and encourage up-to-date screening but may require more visit or charting time or increase testing and referrals. The focused, on the other hand, are expected to better manage patient flow. This study compares resource use ("costs"), clinical quality, patient experience, physician productivity and physician time for focused and max-packing PCPs.

Methods: We used administrative electronic health record (EHR) data and Press-Ganey surveys of a large ambulatory group practice. Our study population included 302 PCPs in 2011-2013 (828 PCP-years). The outcome variables were costs (per-visit PCP evaluation and management [E\&M], and per-episode and annual costs except for inpatient care), clinical quality metrics pertinent to primary care practice, patient experience (patient-reported satisfaction with the care provider and wait time), physician productivity (work RVUs and panel size), and physician EHR open time away from office. All outcome measures were risk-adjusted to account for patient mix in PCP panels. PCPs were classified into three tertiles based on the average number of "other conditions" (identified through diagnosis codes) addressed per acute care episode. We compared PCP-years in the top third (max-packer) and bottom third (the focused).

Results: Max-packing was associated with higher pervisit $\mathrm{E} \& \mathrm{M}$ costs, higher total per-episode costs, but lower annual total costs. Compared to the focused, max-packers had higher scores for clinical quality and overall patient satisfaction, generated more work RVUs per clinical fulltime equivalent, but had more EHR open time away from office and their patients had longer wait times in clinics.

Conclusion: Physician typical practice styles impact multiple dimensions of care delivery. Organizations with potentially competing priorities (affordability, care quality, patient experience, productivity and efficiency) should consider organizational structures and physician incentives with balancing metrics and appropriate risk adjustments that encourage physician behavior achieving primary organizational goals.

\section{P3.04}

Pay-for-Performance Quality Measures: Why Do New Physicians Score Lower?

\section{Su-Ying Liang, ${ }^{1}$ Harold Luft ${ }^{1}$ \\ ${ }^{1}$ Palo Alto Medical Foundation Research Institute, Palo Alto, $C A$}

Background: Public reporting of quality metrics for organizational benchmarking is well-established. Most metrics are well-specified, eg, the proportion of patients with diabetes whose hemoglobin A1c is at target or the proportion of patients in a specific age range having colorectal cancer screening. Patients become eligible for inclusion by developing a condition (diabetes), achieving a specific age (screening), or joining the organization with an undocumented history of prior care. Newly hired physicians may have relatively more of the latter than established physicians with stable panels. We examine whether adjusting for how patients "enter" a panel impacts physician-level quality scores.

Methods: We used administrative and electronic health record data of a large ambulatory group practice. Our study included 389 primary care physicians (PCPs) in 2011-2014 (1,261 PCP-years). We examined adult primary care practice quality metrics for diabetes management and screening services (colorectal/breast/cervical cancer screening and chlamydia). A quality score (number of patients who met the target divided by number eligible) was constructed for each metric. Patients were classified into three groups: 1) new to the organization, 2) newly eligible for screening or newly diagnosed with diabetes, and 3) continuing eligible patients. We compared each set of quality scores for the three patient groups. Adjusted panel-based scores (ratio of observed score to expected score based on patient mix) were compared between newly hired and established PCPs.

Results: Quality scores varied across the patient groups (eg, $57 \%$ vs $76 \%$ vs $63 \%$ for hemoglobin A 1 c control, $37 \%$ vs $44 \%$ vs $73 \%$ for colorectal cancer screening, $60 \%$ vs $44 \%$ vs $50 \%$ for chlamydia screening, at 6th-month postenrollment/diagnosis for new and 6th calendar-month for continuing patients). The "quality gaps" between new and continuing patients were wider shortly after enrollment or diagnosis but narrowed over time. In general, new PCPs had lower scores than established PCPs, but the differences were reduced after this adjustment for patient mix.

Conclusion: Time since a patient is eligible for a quality metric impacts the likelihood of that patient being at goal. When assessing quality metrics across physicians within an organization, the composition of the physicians' panels should be considered.

\section{P3.05 \\ Value of Physician Performance in Diabetes System of Care Among Elderly Medicare Patients: Implications for Pay-for-Performance}

\section{Daniel Maeng, ${ }^{1}$ James Pitcavage, ${ }^{1}$ George Rohrer, ${ }^{1}$ John Bulger ${ }^{1}$}

${ }^{1}$ Geisinger Health System, Danville, PA

Background: While pay-for-performance for physicians is appealing, a direct translation of quality performance into physician payment remains challenging. This study seeks to quantify in dollar terms the value of 
incremental improvements in Geisinger's diabetes system of care (DSC) — ie, an all-or-none "bundle" of 9 diabetes-related performance measures consisting of the following elements: A1C measurement and control; low-density lipoprotein measurement and control; blood pressure measurement; urine protein testing; influenza immunization; pneumococcal immunization; and smoking cessation. Since 2006, Geisinger's primary care physicians (PCPs) have been evaluated and compensated based on their DSC performance.

Methods: This study focused on Medicare Advantage members attributed to Geisinger Clinic's PCPs between January 1, 2006, and September 30, 2014. Claims data were obtained for the subset of the DSC-eligible patients who were Geisinger Health Plan Medicare Advantage members. The key explanatory variable was the DSC performance of the PCP responsible for each patient in the sample, measured as percentages of the PCP's patients meeting all 9 bundle elements. The association between this explanatory variable and per-member-permonth (PMPM) allowed amount was examined using a multivariate regression model.

Results: The results indicate 1-point improvement in the percentage of diabetes patients attributed to a same PCP. Meeting all the DSC elements in a given year - ie, DSC bundle score - was associated with approximately \$4 PMPM (in 2006 dollars; $\mathrm{P}<0.05$ ) reduction in total medical cost (excluding prescription drugs) incurred in the same year, driven mainly by reductions in inpatient cost. Moreover, there is variation in how much each DSC element contributes to the cost reduction; among the 9 elements, urine protein testing and blood pressure measurements were most consistently associated with lower total medical costs.

Conclusion: These findings suggest the DSC may be useful in establishing a feasible pay-for-performance scheme that incentivizes PCPs to improve diabetes care quality.

\section{P3.06}

Primary Care Provider Use Rates of a Clinical Decision Support Tool and Change in Diabetes Performance Measures

\section{JoAnn Sperl-Hillen, ${ }^{1}$ Stephen Asche,${ }^{1}$ Darin Ruanpeng, ${ }^{1}$ Heidi Ekstrom, ${ }^{1}$ Patrick O'Connor ${ }^{1}$ \\ ${ }^{1}$ GHI S\&B for HealthPartners Institute for Education and Research, Bloomington, $M N$}

Background: A previously published randomized controlled trial demonstrated that implementation of an electronic health record-linked personalized clinical decision support (PCDS) tool within primary care clinics improved mean A1c and blood pressure control. We subsequently implemented a modification of our CDS tool for expanded use with high cardiovascular risk adults (CV-PCDS) that also retained the decision support for glycemic control for patients with diabetes. Herein we analyze the association between primary care provider use rates of CV-PCDS with diabetes performance measures in patients with diabetes.

Methods: Using data from a cluster randomized trial in 2012-2014, we analyzed the association of CV-PCDS provider-specific use rates in March 2014 with diabetes performance measures 6 months later, using Pearson correlation coefficients. Performance measures included the proportion of a provider's diabetes patients who (a) achieved A $1 \mathrm{c}<8 \%$, and (b) achieved a composite measure of optimal diabetes care that required simultaneous achievement of A1c $<8 \%$, systolic blood pressure $<140 \mathrm{~mm} \mathrm{Hg}$, low-density lipoprotein cholesterol $<100 \mathrm{mg} / \mathrm{dl}$, non-tobacco user, and ASA (aspirin) use for secondary prevention.

Results: Providers $(\mathrm{N}=43)$ used the CV-PCDS tool at a mean of $82.1 \%$ of targeted encounters of adults with high cardiovascular risk (range across providers: $36.0 \%$ to $100 \%$ of encounters). The mean percentage of the diabetes subgroup who achieved A $1 \mathrm{c}<8 \%$ was $73.7 \%$, and the percentage of patients who achieved the optimal diabetes care goal was 46.8\%. Pearson correlation coefficients between CV-PCDS provider use rates in March 2014 and A1c and optimal diabetes care performance measures in August 2014 were $0.16(\mathrm{P}=0.31)$ and $0.24(\mathrm{P}=0.12)$, respectively.

Conclusion: In this high-performing health care system with high CV-PCDS use rates, there was a positive but nonsignificant association of provider use of the CVPCDS tool and provider-level quality of diabetes care 6 months later. The generalizability of this finding to lowerperforming care systems, and to providers with lower baseline quality of diabetes care remains to be determined.

\section{P3.07}

Impact of Improving Diabetes Care on QualityAdjusted Life Expectancy and Costs: A 40-Year Perspective

Patrick O'Connor, ${ }^{1}$ Todd Gilmer, ${ }^{1}$ JoAnn Sperl-Hillen, ${ }^{1}$ Heidi Ekstrom, ${ }^{1}$ Lauren Crain ${ }^{1}$

${ }^{1}$ GHI S\&B for HealthPartners Institute for Education and Research, Bloomington, $M N$

Background: There has been a trend toward better management of glucose, lipids, blood pressure, smoking and other aspects of diabetes care in the last decade. The goals of this study are to assess changes in quality of diabetes care over a 14-month period of time in a large, multispecialty U.S. medical group, to quantify treatment costs associated with the improved care, and to estimate the impact of care improvement on long-term costs and quality-adjusted life expectancy.

Methods: Study subjects included 7,054 persons with diabetes, age 40-75 years, who at baseline had one or more of: systolic blood pressure $>140 \mathrm{mmHg}$, low-density 
lipoprotein cholesterol $>129 \mathrm{mg} / \mathrm{dL}$, or current smoking status. We quantified their clinical status including A1c, blood pressure, lipids and smoking status both at baseline and after a median 14-month follow-up period. We similarly quantified visit frequency and medication use and associated costs in the year prior and year following the baseline visit. We employed these clinical risk factors and observed costs as data inputs into a log-term simulation model of diabetes outcomes - the United Kingdom Prospective Diabetes Study Outcomes Model (Version 2) — in order to estimate changes in quality-adjusted life years (QALYs) and costs associated with changes in clinical care, projected over a 40-year time period. We applied costs of complications that were derived from a previous study within this health system. We then estimated the cost per QALY gain for these adult diabetes subjects who are experiencing better clinical care over time.

Results: Observed improvements in clinical care significantly increased expected QALY from 10.83 to 11.06 , for a gain in 0.22 QALY. Incremental costs associated with outpatient visits and intensification of pharmacotherapy were $\$ 167$ per year and $\$ 2,323$ over the study period. Total costs increased by $\$ 4,453$. Cost per QALY was estimated to be $\$ 19,866$. Sensitivity analysis indicated that estimates of cost per QALY were more favorable in simulations with longer follow-up periods and in simulations that more narrowly targeted blood pressure control among those with high blood pressure at baseline.

Conclusion: Observed improvements in diabetes care over a recent 14-month period of time are sufficient to significantly improve clinical and health outcomes. The cost-effectiveness of the slightly more intensive diabetes care provided appears to be satisfactory using standard thresholds for cost per QALY, both in the base case and across a range of sensitivity analysis scenarios. However, improvements in diabetes care are not cost saving from the point of view of the payer.

\section{P3.08}

Clinical Decision Support Impact on Overuse and Underuse of Aspirin for Primary Prevention of Cardiovascular Events

\section{Patrick O'Connor, ${ }^{1} \quad$ JoAnn Sperl-Hillen, ${ }^{1}$ Karen Margolis, ${ }^{1}$ Lauren Crain, ${ }^{1}$ Heidi Ekstrom ${ }^{1}$ \\ ${ }^{1}$ GHI S\&B for HealthPartners Institute for Education and Research, Bloomington, MN}

Background: The U.S. Preventive Services Task Force (USPSTF) recommends aspirin for primary prevention of atherosclerotic vascular disease (ASCVD) when the ASCVD benefit outweighs the risk of gastrointestinal hemorrhage. The complexity and time required to assess aspirin risks and benefits can result in overuse and underuse of aspirin.

Methods: As part of a National Institutes of Health-funded study to lower ASCVD risk, we implemented electronic clinical decision support (CDS) algorithms to guide aspirin use based on USPSTF criteria and major bleeding risks. Baseline data was collected for whether aspirin was algorithmically recommended for all patients at their first eligible primary care encounter in 20 clinics over 2012 2014. The analysis excluded patients with congenital heart disease and included 6,651 adults with diabetes (mean age: 55.6 years; mean 10-year ASCVD risk: 27.8\%) and 11,682 adults meeting prespecified criteria for high ASCVD risk without diabetes (mean age: 58.4 years; mean 10-year ASCVD risk: 24.7\%). Overuse and underuse was determined by comparing concordance with (a) aspirin recommendations, and (b) documented aspirin use.

Results: The CDS recommended aspirin for 4,139 (63.1\%) patients with diabetes and 8,722 (74.7\%) without diabetes. Among patients with aspirin recommended, aspirin was not used in 829 of $4,139(20 \%)$ with diabetes and 6,493 of 8,722 (74.4\%) without diabetes (underuse). Among patients for whom the CDS did not recommend aspirin, aspirin was used in 1,448 of $2,969(59.8 \%)$ with diabetes and 1,021 of 2,960 (34.4\%) without diabetes (overuse).

Conclusion: Those with diabetes who were likely to benefit from aspirin use had higher aspirin use rates (less underuse) than similar high-cardiovascular-risk patients without diabetes. However, those with diabetes who were unlikely to benefit from aspirin based on USPSTF criteria and bleeding risks also had higher aspirin use rates (more overuse) than patients without diabetes. Strategies to ensure greater evidence-based use of aspirin, such as providing electronic clinical decision support, may help providers more accurately assess individualized risks and benefits of aspirin.

\section{P3.09}

Can Prioritized Clinical Decision Support in Primary Care Reduce Cardiovascular Risk?

Patrick O'Connor, ${ }^{1}$ JoAnn Sperl-Hillen, ${ }^{1}$ Karen Margolis, ${ }^{1}$ Lauren Crain, ${ }^{1}$ William Rush, ${ }^{1}$ Heidi Ekstrom, ${ }^{1}$ Jerry Amundson, ${ }^{1}$ Rashmi Sharma, ${ }^{1}$ Deepika Appana $^{1}$

${ }^{1}$ GHI S\&B for HealthPartners Institute for Education and Research, Bloomington, $M N$

Background: The objective of this project was to develop and implement sophisticated point-of-care electronic health record (EHR)-based clinical decision support that (a) identifies, and (b) prioritizes all available evidence-based treatment options to reduce a given patient's cardiovascular risk (CVR).

Methods: We randomized 19 primary care clinics with 102 primary care providers and 39,025 adults with diabetes, cardiovascular disease or 10-year American College of Cardiology/American Heart Association (ACC/AHA) reversible $\mathrm{CVR} \geq 10 \%$ into one of two experimental 
conditions. Group 1 included 10 clinics that received the CV Wizard; Group 2 included 9 usual care clinics. The study formally tested the hypothesis that after control for baseline CVR, postintervention ACC/AHA 10-year CVR (risk of fatal or nonfatal heart attack or stroke) will be significantly better in Group 1 than Group 2 in the postintervention period.

Results: The CV Wizard system was integrated successfully into the workflow of primary care visits; use rates at targeted visits in intervention clinics ranged from $44 \%$ to $77 \%$ and improved over time. In the high reversible CV risk sample $(\mathrm{n}=7,595), 10$-year ACC/AHA CVR declined by $-0.030 \%$ per visit in the control group $(\mathrm{P}=0.001)$ and by $-0.46 \%$ per visit in the intervention group $(\mathrm{P}=0.28)$; this difference in annual rate of change in CVR was statistically significant and favored the intervention group $(\mathrm{P}<0.001)$. In the diabetes sample $(n=5,510)$, the observed change in 10 year ACC/AHA CVR was $+0.06 \%$ per visit in the control group $(\mathrm{P}=0.56)$ and by $-0.23 \%$ per visit in the intervention group ( $\mathrm{P}<0.03$ ); this difference in rate of change in CVR was statistically significant and favored the intervention group $(\mathrm{P}=0.049)$. The predicted annual change in $\mathrm{CVR}$ was $+0.91 \%$ in the control group $(\mathrm{P}=0.16)$ and $+0.38 \%$ in the intervention group $(\mathrm{P}=0.55)$, this difference in annual rate of change in CVR was not statistically significant $(\mathrm{P}=0.56)$. In the cardiovascular disease sample $(\mathrm{n}=2,078), 10$-year ACC/AHA CVR change over visits $(\mathrm{P}=0.42)$, and over time $(\mathrm{P}=0.92)$ was not significant when comparing intervention and usual care clinics.

Conclusion: The overall pattern of change in CVR, whether measured by visit or time, was consistent with the assertion that CVR decreased at a faster rate (or increased at a slower rate) in the Wizard intervention clinics relative to control clinics for those with diabetes or high reversible CVR, but not for those with known cardiovascular disease. The difference in trajectories reached statistical significance over the course of visits among diabetes patients, and over time among patients with high reversible CVR. Use rates and primary care provider satisfaction with the CV Wizard were very high, and economic analysis suggests the improved care is cost-effective. Based on these and other research results, the CV Wizard clinical decision support system is currently being used at three large health care delivery systems in four states that provide care to $1,500,000$ patients.

P3.10

Taking Action on Overuse: Implementing an Action-Planning Framework to Engage Providers

Michael Parchman, ${ }^{1}$ Nora Henrikson, ${ }^{1}$ Paula Blasi, ${ }^{1}$ Diana Buist, ${ }^{1}$ Brian Austin, ${ }^{1}$ Robert Penfold ${ }^{1}$

${ }^{1}$ Group Health Research Institute, Seattle, WA

Background: Unnecessary care contributes to high costs and places patients at risk of harm. While most providers support reducing low-value care, changing established practice patterns is difficult and requires active engagement in sustained behavioral, organizational and cultural change. Here we describe an action-planning framework to engage providers in reducing overused services.

Methods: The framework is informed by a comprehensive review of social science theory and literature, published reports of successful and unsuccessful efforts to reduce low-value care, and interviews with innovators of valuebased care initiatives in 23 health care organizations across the United States. A multistakeholder advisory committee provided feedback on the framework and guidance on optimizing it for use in practice.

Results: The framework (www.takingactiononoveruse. org/framework/) describes four conditions necessary for accelerating change: prioritize addressing low-value care; build a culture of trust, innovation and improvement; establish shared language and purpose; and commit resources to measurements. When these conditions are present, they catalyze productive sense-making conversations between providers, between providers and patients, and among members of the health care team about the potential for harm from overuse and reflection on current frequency of use. Through these conversations providers, patients and team members think together as a group, learn how to coordinate individual behaviors and jointly develop possibilities and plans for coordinated action around specific areas of overuse. Key changes used by innovative health care organizations to create conditions for change and foster sense-making conversations are described along with examples of activities used to implement these changes. Early work with health care systems to utilize the framework in three settings will be described: an outpatient specialty care clinic, an acute care inpatient setting and a statewide regional cooperative.

Conclusion: Organizational efforts to engage providers in value-based care are focused on creating conditions for productive sense-making conversations that lead to change. Organizations can use this framework to enhance and strengthen provider engagement efforts to do less of what potentially harms and more of what truly helps patients.

\section{P3.11}

System-Level Barriers to Follow-up Colonoscopy Completion After Positive Fecal Test: Interviews With Gastroenterologists and Staff

Jennifer Schneider, ${ }^{1}$ Jennifer Rivelli, ${ }^{1}$ Basak Gokcora, ${ }^{1}$ Keshia Bigler, ${ }^{1}$ Amanda Petrik, ${ }^{1}$ Beverly Green, ${ }^{2}$ Gloria Coronado ${ }^{1}$

${ }^{1}$ Kaiser Permanente Center for Health Research, Portland, OR; ${ }^{2}$ Group Health Research Institute, Seattle, WA

Background: Despite the efficacy of colorectal cancer (CRC) screening, many adults are not screened at recommended intervals or screened at all. Federally 
qualified health centers (FQHCs) serve a unique patient population that often experience barriers to CRC screening. Yearly fecal testing and colonoscopy followup for positive test results can reduce CRC incidence and mortality. However, many patients with positive fecal test results forgo follow-up colonoscopy, nullifying the potential benefits of fecal testing.

Methods: As part of the Strategies and Opportunities to STOP Colon Cancer in Priority Populations (STOP CRC) study, we qualitatively explored factors associated with referral to and completion of follow-up colonoscopy after a positive fecal test. Through interviews with gastroenterologist (GI) providers and office staff, we sought to elucidate systemlevel barriers to colonoscopy completion and identify areas for improvement for patients referred from FQHCs. Specialists and their staff were recruited via email, and interviews were conducted in-person or by phone using an interview guide. Interviews were recorded, transcribed, coded and content-analyzed by trained qualitative staff, resulting in refined themes.

Results: We completed 15 in-depth interviews with GI providers $(\mathrm{n}=8)$ and office staff $(\mathrm{n}=7)$. The most frequently cited challenges by all interviewees related to increase in demand following the Affordable Care Act, complexity and time delay issues with insurance requirements, and inadequate staffing at the specialist office. GI providers emphasized capacity issues, including long wait times and limited appointment options. Office staff highlighted lack of complete referral and medical review documentation and language barriers. Improving communication and electronic referral documentation between the $\mathrm{FQHC}$ and GI office was identified by participants as vitally important. GI providers also advised on the need to reduce no-show rates and improve scheduling; and office staff desired preparation instructions in additional languages and skilled interpreters to attend the colonoscopy procedure with the patient.

Conclusion: The life-saving benefits of CRC screening can be maximized by identifying challenges to followup colonoscopy after a positive fecal test. Uncovering system-level issues and possible areas for improvement in referral coordination and procedure completion can further contribute to increasing CRC screening rates for patients receiving care in $\mathrm{FQHCs}$.

\section{P3.12}

\section{Reducing Opioid Exposure Following Hip and Knee Surgery: Results of a Randomized, Pragmatic, Pharmacist-Led Intervention}

David Smith, ${ }^{1}$ Jennifer Kuntz, ${ }^{1}$ Eric Johnson, ${ }^{1}$ Jennifer Schneider, ${ }^{1}$ Xiuhai Yang, ${ }^{1}$ Lynn DeBar ${ }^{1}$

${ }^{1}$ Kaiser Permanente Center for Health Research, Portland, OR

Background: Opioids have a role in managing pain for orthopaedic patients, but there is compelling evidence that this exposure should be kept as short as possible. Setting patient expectations for pain and its treatment has been recommended for orthopaedic surgeons as part of minimizing opioid exposure.

Methods: Patients scheduled to undergo total hip arthroplasty (THA) or total knee arthroplasty (TKA) were randomized, weekly, to usual care or intervention. Intervention patients received mailed materials 2 weeks before and after surgery, plus telephone intervention from specially trained pharmacists if they filled opioid prescriptions in the 28-90 days following surgery. Materials were developed using qualitative methods involving patients and orthopaedic clinicians. In order to direct resources toward the riskiest patients, we ranked patients according to predicted risk of persistent opioid use and selected the top $60 \%$. Our primary outcome was the morphine equivalents (MEQ) dispensed in the 90 days following surgery, modeled using a natural $\log$ transformation (lnMEQ). We report the relative percentage decrease in geometric means between study groups, with a P-value $<0.05$ cut-point for statistical significance. Our a priori analytic plan specified testing for effect modification on treatment group by: 1) THA or TKA, 2) baseline quartile of opioid use, and 3) quartile of predicted opioid persistence. Informed consent was waived, consistent with pragmatic trial principles.

Results: A total of 561 patients were randomized (286 usual care, 118 THA, 275 intervention, 107 THA); mean age was 66 years and $60 \%$ were female. The intervention failed to reduce $\operatorname{lnMEQ}$ in the entire surgical population $(\mathrm{P}>0.50)$, but the intervention reduced $\ln M E Q$ by a larger percentage in the THA patients than the TKA patients $(\mathrm{P}<0.003)$. Among THA patients, those randomized to intervention used $62 \%$ less MEQ $(\mathrm{P}<0.003)$ than usual care. We found no statistically significant difference in opioid use among TKA patients $(\mathrm{P}>0.1)$. We found no effect modification by baseline opioid use or predicted opioid persistence.

Conclusion: Our pharmacist-led intervention to reduce opioid use had clinically meaningful impact on the use of opioids in the 90 days following THA, but not TKA.

\section{P3.13}

\section{A Clinical Decision Support System Promotes Shared Decision-Making and Cardiovascular Risk Factor Management}

\section{JoAnn Sperl-Hillen, ${ }^{1}$ Lauren Crain, ${ }^{1}$ Heidi Ekstrom, ${ }^{1}$ Karen Margolis ${ }^{1}$ \\ ${ }^{1}$ GHI S\&B for HealthPartners Institute for Education and Research, Bloomington, $M N$}

Background: CV Wizard is a web-based electronic health record-integrated point-of-care clinical decision support (CDS) system that presents personalized cardiovascular (CV) risk information to providers and patients in both a low numeracy visual format and a high numeracy quantitative format. Herein we report primary care 
provider perspectives on how this CDS system affected shared decision-making and $\mathrm{CV}$ risk factor management.

Methods: Twenty clinics were randomized to either usual care or use of the CDS system for adults with diabetes, heart disease or high reversible CV risk. The CDS system targeted $20 \%$ of office visits and was used at $70 \%-80 \%$ of targeted visits over a 2 -year period. Consented providers $(\mathrm{n}=102)$ were surveyed at baseline and 18 months after implementation. Corrected survey response rates were $90 \%$ at baseline and $82 \%$ at follow-up. Generalized linear mixed models were used to compare usual care and CDS responses to common questions at baseline and follow-up, and CDS users were queried on their perceptions of the CDS system at follow-up only.

Results: Compared to usual care providers, those in the CDS group reported increased follow-up rates of $\mathrm{CV}$ risk calculations while seeing patients ( $73 \%$ vs $28 \%, \mathrm{P}=0.006$ ), being better prepared to discuss $\mathrm{CV}$ risk reduction priorities with patients ( $98 \%$ vs $78 \%, \mathrm{P}=0.03)$, providing accurate advice on aspirin for primary prevention $(75 \%$ vs $48 \%$, $\mathrm{P}=0.02$ ), and more often discussing $\mathrm{CV}$ risk reduction ( $60 \%$ vs $30 \%, \mathrm{P}=0.06$ ). CDS users reported that the CDS system improved CV risk factor control (98\%), saved time when talking to patients about CV risk reduction (93\%), efficiently elicited patient treatment preferences $(90 \%)$, was useful for shared decision-making (95\%), influenced treatment recommendations $(89 \%)$ and helped initiate $\mathrm{CV}$ risk discussions (94\%); $85 \%$ of providers reported that their patients liked CV Wizard.

Conclusion: The CV Wizard CDS system was successfully integrated into the workflow of primary care visits with high sustained use rates, high primary care provider satisfaction, high patient satisfaction, and positive impacts on providerreported clinical processes related to $\mathrm{CV}$ risk factor management.

\section{P3.14}

\section{Aspirin for Primary Prevention of Atherosclerotic Cardiovascular Disease: Challenges to Appropriate Use}

JoAnn Sperl-Hillen, ${ }^{1}$ Patrick O'Connor, ${ }^{1}$ Jay Desai, ${ }^{1}$ Karen Margolis, ${ }^{1}$ Heidi Ekstrom, ${ }^{1}$ Lauren Crain ${ }^{1}$

${ }^{I}$ GHI S\&B for HealthPartners Institute for Education and Research, Bloomington, $M N$

Background: Aspirin use for primary prevention of atherosclerotic cardiovascular disease (ASCVD) should be highly individualized in order to accurately balance benefits and risks. There are now practical approaches for clinicians to calculate ASCVD and bleeding risks using web-based tools and mobile apps to facilitate good decision-making, but their lack of integration with the electronic health record (EHR) and need for extensive data input are barriers to use by busy clinicians.
Methods: As part of a clinic randomized trial with 20 primary care clinics, we developed and tested an EHR-integrated web-based clinical decision support (CDS) system that provided individualized aspirin recommendations to patients and clinicians using riskbenefit calculations. During the 18-month intervention, aspirin recommendations were printed for patients and providers at $75 \%$ of eligible encounters. We evaluated the effects of the intervention on rates of appropriate primary prevention aspirin use among 3,958 patients with diabetes and 7,000 patients without diabetes, aged $40-75$ years, with uncontrolled cardiovascular disease risk factors.

Results: At baseline, among patients using aspirin, it was not recommended (overused) for 840 of 1,474 (57\%) patients with diabetes and 564 of 1,659 (34\%) without diabetes. Of patients not using aspirin, it was recommended (underused) by 522 of 2,484 (21\%) patients with diabetes and 4,006 of $5,371(75 \%)$ without diabetes. At the last follow-up visit, no significant differences were noted in aspirin use patterns for patients with diabetes. However, among patients without diabetes who were "underusing" aspirin at baseline, $12.9 \%$ were using aspirin in CDS clinics compared to $10.4 \%$ in control clinics $(\mathrm{P}=0.03)$. Among patients who were "overusing" aspirin at baseline, $4.1 \%$ had discontinued using aspirin in CDS system clinics compared to $7.9 \%$ in control clinics $(\mathrm{P}=0.06)$.

Conclusion: Patterns of appropriate aspirin use are different among patients with and without diabetes, with overuse being more common in diabetes and underuse more common in those without diabetes. Our study results suggest that the use of accurate CDS by clinicians and patients improve overall concordance with aspirin. A better understanding of how best to present understandable riskbenefit information to providers and patients is needed.

\section{CHILD AND ADOLESCENT HEALTH}

P4.01

Results of a Cluster-Randomized Trial Testing the Effects of TeenBP, an Electronic Health Record-Based Clinical Decision Support Tool, on Recognition of Adolescent Hypertension

Elyse Kharbanda, ${ }^{1}$ Stephen Asche, ${ }^{1}$ James Nordin, ${ }^{1}$ Alan Sinaiko, ${ }^{2}$ Heidi Ekstrom, ${ }^{1}$ Nancy Sherwood, ${ }^{1}$ Patricia Fontaine, ${ }^{1}$ Steven Dehmer, ${ }^{1}$ Jerry Amundson, ${ }^{1}$ Deepika Appana, ${ }^{1}$ Patrick O'Connor ${ }^{1}$

${ }^{1}$ HealthPartners Institute, Minneapolis, MN; ${ }^{2}$ Department of Pediatrics, University of Minnesota, Minneapolis, MN

Background: Hypertension occurs in 1\%-3\% of adolescents and is associated with long-term cardiovascular morbidity. Although blood pressure (BP) is routinely measured, hypertension in adolescents is often missed during 
outpatient visits. This study sought to evaluate whether "TeenBP," an electronic health record (EHR)-linked, web-based clinical decision support tool, could improve recognition and early management of hypertension in adolescents.

Methods: We randomized 20 primary care clinics to receive the $\mathrm{CDS}$ or to continue usual care. At intervention sites, TeenBP was activated at the point of care when a BP was elevated. TeenBP graphically displayed systolic and diastolic BP values and percentiles over the prior 2 years, identified patients meeting criteria for hypertension (3 or more BPs $\geq 95$ th percentile), provided a summary of comorbidities and medications that may affect BP, and offered patient-specific order sets. Hypertension recognition, within 6 months of meeting criteria, was defined as adding hypertension or elevated BP (EBP) to the problem list or clinical notes, indicating hypertension or EBP as an outpatient visit diagnosis, prescribing a medication to lower $\mathrm{BP}$, or diagnostic testing related to hypertension, and was identified through chart review. Generalized linear mixed models were used to test the effect of the intervention.

Results: Among 21,618 patients aged 10-17 years with a primary care visit at the 20 study clinics over one year (May 2014-April 2015), 315 (1.5\%) met criteria for new-onset hypertension. Most BPs were modestly elevated $(<99$ th percentile). Clinical recognition of hypertension within 6 months occurred for $36.2 \%(27.7 \%-45.8 \%)$ of patients in usual care clinics and $68.0 \%(56.6 \%-77.6 \%)$ in the CDS clinics $(\mathrm{P}=0.0003)$. Clinical recognition of hypertension in the TeenBP clinics was most often met by adding hypertension or EBP as an outpatient visit diagnosis $(52.2 \%)$ or to the text of the clinical note $(52.4 \%)$. Within 6 months of meeting criteria for hypertension, less than $10 \%$ of TeenBP or usual care subjects had an echocardiogram or renal ultrasound and only one patient initiated an antihypertensive medication.

Conclusion: We observed a large and statistically significant beneficial effect of this clinical decision support system on recognition of new-onset hypertension, without increasing diagnostic workups or early initiation of antihypertensive medication.

\section{P4.02}

Development and Validation of a Risk Equation for Appendicitis in Children Presenting With Abdominal Pain

Gabriela Vazquez-Benitez, ${ }^{1}$ Elyse Kharbanda, ${ }^{1}$ Dustin Ballard, ${ }^{2}$ David Vinson, ${ }^{2}$ Richard Bachur, ${ }^{3}$ Uli Chettipally, ${ }^{2}$ Mamata Kene, ${ }^{2}$ Patrick O'Connor, ${ }^{1}$ Steven Dehmer, ${ }^{1}$ Heidi Ekstrom, ${ }^{1}$ Peter Dayan, ${ }^{4}$ Nathan Kuppermann, ${ }^{5}$ Anupam Kharbanda ${ }^{6}$
${ }^{1}$ HealthPartners Institute, Minneapolis, MN; ${ }^{2}$ Kaiser Permanente Northern California, Oakland, CA; ${ }^{3}$ Boston Children's Hospital, Boston, MA; ${ }^{4}$ Columbia University, New York, NY; ${ }^{5}$ University of California, Davis, Sacramento, CA; ${ }^{6}$ Children's Hospital and Clinics Minnesota, Minneapolis, MN

Background: Appendicitis is a common surgical emergency in children, yet the diagnosis remains challenging. A widely used risk score, the Pediatric Appendicitis Score, is not sufficiently sensitive or specific to be used alone, with many patients classified as "intermediate risk." Goals of this study were to develop and validate an improved appendicitis risk calculator for children with acute abdominal pain to aid in clinical decision-making.

Methods: We developed our risk calculator using data from a multicenter cohort of children 5 to 18 years old presenting to the emergency department (ED) with acute abdominal pain. We validated the risk calculator in two independent cohorts with similar enrollment criteria. Patient history, physical examination and laboratory data were prospectively recorded by clinicians during ED visits. Appendicitis was confirmed by pathology reports and follow-up telephone survey. Variables evaluated for inclusion in the risk calculator were: age, sex, pain duration, pain with walking, migration of pain, temperature, heart rate, guarding, maximal tenderness in right lower quadrant, white blood cell count, and absolute neutrophil count. A step-wise regression approach was followed to select the best model, using Akaike information criteria and the C-statistic. We forced inclusion of age and sex, including first-order interaction terms. Laboratory values were evaluated for nonlinear associations with appendicitis, and a two-step linear association was included. Validation included calibration and discrimination analyses.

Results: The development sample included 2,423 children, of whom $40 \%$ had appendicitis; the validation sample included 1,426 , and $35 \%$ had appendicitis. Our final risk calculator included sex, age, duration of pain, guarding, migration of pain and absolute neutrophil count. In the validation sample, calibration plot and Hosmer and Lemeshow test $(\mathrm{P}<0.0001)$ showed high calibration and a high discrimination $(\mathrm{C}$-statistic $=0.86)$. Among $248(17 \%)$ patients in the validation sample at $<5 \%$ predicted risk, we observed 4\% had appendicitis. Of an additional $318(22 \%)$ patients with predicted risk $5 \%$ to $<15 \%$, appendicitis occurred in $8 \%$. Of $48(3.4 \%)$ patients in the validation sample at predicted risk of $>90 \%, 96 \%$ had appendicitis.

Conclusion: Our validated pediatric appendicitis risk calculator can accurately quantify risk for appendicitis and can identify children with acute abdominal pain at high or low risk for appendicitis. 


\section{CHRONIC CONDITIONS, DIABETES, OBESITY, CARDIOVASCULAR DISEASE}

P5.01

\section{Bisphosphonates Use and Risk of Hip Fractures}

Sarah Sharman Moser, ${ }^{1}$ Inbal Goldshtein, ${ }^{1}$ Jingbo Yu, ${ }^{2}$ Clara Weil, ${ }^{1}$ Sophia Ish-Shalom, ${ }^{3}$ Vanessa Rouach, ${ }^{1}$ Varda Shalev, ${ }^{4}$ Gabriel Chodick ${ }^{4}$

${ }^{1}$ Maccabi Healthcare Services, Tel Aviv, Israel; ${ }^{2}$ Center for Observational Research and Real-World Evidence, Merck \& Company Inc., Kenilworth, NJ; ${ }^{3}$ Elisha Hospital, Haifa, Israel; ${ }^{4}$ Maccabi Healthcare Services \& Tel Aviv University, Tel Aviv, Israel

Background: Osteoporosis affects 200 million women worldwide, and fractures resulting from bone fragility are a major complication. Bisphosphonates slow down bone resorption by osteoclasts and have been shown to lower the risk of hip fractures among patients with osteoporosis in clinical trial settings. Most clinical guidelines recommend pharmacological treatment for postmenopausal women with an existing fracture or bone density T-score of -2.5 or lower. These guidelines were based on randomized controlled trials that typically lasted 3-4 years, although some of these studies have been extended for up to 10 years. We undertook this study to examine the association between adherence with bisphosphonate therapy and long-term risk of hip fracture, using up to 14 years of retrospective observation.

Methods: Included in the present nested case-control study were osteoporotic women $(\mathrm{N}=14,357)$ who initiated bisphosphonate therapy between the years 2000 and 2010 and were retrospectively followed for incident hip fracture through November 2014. All cases of primary hip fracture within the cohort $(n=411)$ were individually matched with 411 controls based on age ( \pm 1 year) and calendar year at therapy initiation as well as follow-up duration ( \pm 1 year). For each study participant, the total dispensed packs of bisphosphonates during the follow-up period were counted. Results: Compared to patients discontinuing therapy within 1 month, therapy with bisphosphonates for 1 to 3 years and 3 to 6 years was associated with adjusted odds ratios (and 95\% confidence intervals) for hip fractures of $0.48(0.27-0.86)$ and $0.40(0.22-0.73)$, respectively. Comparable odds ratios were calculated for patients covered for longer periods $0.36(0.17-0.76)$ for 6 to 8 years, and $0.57(0.27-1.21)$ for 8 years or more.

Conclusion: Results suggest that 3 years or more of bisphosphonates use is associated with a substantially lower risk of primary hip fracture compared to nonpersistent patients. The results do not indicate further reduction in risk among patients treated for 8 years or more.
P5.02

How Do Patients With Chronic Conditions Prefer to Get Health Information and Advice? Results From the 2014 Kaiser Permanente Northern California Member Health Survey

\author{
Nancy P. Gordon, ${ }^{1}$ Teresa Y. Lin ${ }^{1}$ \\ ${ }^{1}$ Kaiser Permanente Division of Research, Oakland, CA
}

Background: Management of chronic conditions requires patient engagement in health promoting self-care, which ongoing health education, advice and reinforcement may enhance. Information about sociodemographic differences in patient preferences for patient health information/advice services (HIA), especially regarding web-based/digital (eHIA) versus more traditional modalities, can inform development and delivery of interventions.

Methods: Weighted data from the 2014 Kaiser Permanente Northern California Member Health Survey for 2,780 adults, aged 40-85 years, with diabetes, hypertension, coronary artery disease and/or history of stroke were used to assess ability to use digital technology (internet, email, text messages, smartphone), prior-year use of eHIA and traditional health education resources, and preferred methods for getting health information and advice (restricted to those who indicated $\geq 1$ method). Data were analyzed by age group.

Results: Use of digital technologies, analyzed for ages 40-64, 65-69, 70-74, 75-79 and 80-85, declined with age (internet and email: 94\%, 92\%, 85\%, 71\%, 59\%; text messages: $74 \%, 54 \%, 47 \%, 33 \%, 27 \%$; smartphone: $64 \%, 46 \%, 32 \%, 33 \%, 27 \%$ ). HIA use was analyzed for ages $40-64,65-69,70-74$ and $75-85$. More than 70\% (no age differences) had obtained HIA in the past year; $50 \%$ had used eHIA, but this declined with age $(51 \%, 57 \%$, $50 \%, 38 \%)$. Of those interested in $\geq 1$ HIA modality: $55 \%(59 \%, 58 \%, 48 \%, 38 \%)$ were interested in $\geq 1$ eHIA modality; $48 \%(51 \%, 53 \%, 45 \%, 36 \%)$ in reading information on websites; $34 \%(35 \%, 40 \%, 32 \%, 28 \%)$ in emailed newsletters; $34 \%$ (range: $39 \%-23 \%$ ) in emailed messages; $18 \%(21 \%, 20 \%, 15 \%, 6 \%)$ in online videos; $16 \%(22 \%, 15 \%, 8 \%, 4 \%)$ in health apps; $11 \%(14 \%$, $9 \%, 9 \%, 3 \%$ ) in online programs; $8 \%$ (range: $9 \%-3 \%$ ) in webinars; $6 \%$ in podcasts; and 3\% in chat rooms. Interest in more traditional HIA modalities was $41 \%$ individual counseling (30\% in-person, $18 \%$ phone-based), $37 \%$ print materials, $22 \%$ workshops and $15 \%$ classes/groups. Minimal gender differences were observed.

Conclusion: While most patients with chronic conditions use internet and email, many, especially older seniors, still prefer using more traditional modalities for obtaining health information and advice. 
P5.03

\section{Postdeployment Mental Health Status and Obesity Among a Multigenerational Sample of U.S. Veterans}

Johanna Hyacinthe, ${ }^{1}$ Stuart Hoffman, ${ }^{1}$ Thomas Urosevich, ${ }^{1}$ H. Lester Kirchner, ${ }^{1}$ Richard Adams, ${ }^{2}$ Charles Figley, ${ }^{3}$ Joseph Boscarino ${ }^{1}$

${ }^{1}$ Geisinger Health System, Danville, PA; ${ }^{2}$ Kent State University, Kent, OH; ${ }^{3}$ Tulane University, New Orleans, LA

Background: Since history of posttraumatic stress disorder (PTSD) and depression have been associated with obesity in previous studies, the objective of the current study was to assess if these disorders were associated with obesity among formerly deployed U.S. veterans.

Methods: We surveyed a random sample of U.S. veterans who were patients in a large non-Veterans Affairs (VA) multihospital system located in central and northeastern Pennsylvania to assess their mental health, physical health and obesity status. Obesity was defined as having a body mass index $>30$. Our hypothesis was that mental health status, including having PTSD or depression, was associated with obesity.

Results: Of 1,289 veterans surveyed (response rate $\sim 60 \%), 53.6 \%$ were from the Vietnam era, $95.0 \%$ were male, $54.5 \%$ were $\geq 65$ years old, $95.7 \%$ were white race, and $26.9 \%$ were recent National Guard or Reserve service members. Altogether, $44.3 \%$ (95\% confidence interval: 41.5-47.1) of veterans were classified as obese. Study variables associated with obesity included nonwhite race, not having a college degree, having a lower income, reporting lower unit support during deployment, currently using the VA system, having applied for or received VA disability, having ever used mental health services, currently using psychotropic medications, reporting poor/fair health status, and reporting pain in the past month $(\mathrm{P}<0.05$ for all). Contrary to our hypothesis, neither having PTSD nor depression were associated with obesity among veterans in the current study. In multivariable analyses that adjusted for age, gender, education, combat exposure, life stressors and social support, no association was found between mental health status and obesity. The best predictors of current obesity among veterans was having a college education (odds ratio: $0.53 ; \mathrm{P}<0.001$ ), suggesting that college was protective, and reporting poor or fair health status (odds ratio: $1.51 ; \mathrm{P}=0.001$ ).

Conclusion: These preliminary data do not support the link between mental health status and obesity among deployed U.S. veterans. Further research that examines obesity, and eating disorders, among formerly deployed veterans is planned.
P5.04

Specificity of the Framingham Heart Failure Signs and Symptoms: Bridging the Gap Between Epidemiological Research and Clinical Practice

\author{
Heather Law, ${ }^{1}$ Sanjoy Dey, ${ }^{2}$ Walter Stewart ${ }^{1}$ \\ ${ }^{1}$ Sutter Health, Walnut Creek, CA; ${ }^{2} I B M$, Yorktown Heights, NY
}

Background: While the Framingham Heart Failure Signs and Symptoms (FHFSS) have been around for decades, little is known about the specificity of the relation between presence of each FHFSS and early detection of heart failure. The FHFSS continue to be used in epidemiological research; however, their clinical relevance has been criticized. We examined variation in the ability of individual FHFSS to predict heart failure diagnosis by assertion or denial of each feature, by counts, and by a composite score.

Methods: We extracted electronic health record data from 2001 to 2010 from a single health system. A total of 1,684 incident heart failure cases were identified, and 13,525 matched controls were selected from the same primary care practices. We performed LASSO logistic regression analysis to determine which FHFSS are the strongest predictors of heart failure. We explored predictive value by observation window (12 vs 24 months) and by prediction window (6, 12,18 and 24 months). FHFSS score statistics were used to determine if prediction was improved and to determine if changes in score over time improved the model performance. Results: Our findings indicate that: 1) Less features yield better prediction performance (LASSO model), with the following FHFSS features being most predictive: negative/ positive acute pulmonary edema, negative/positive bilateral ankle edema, positive dyspnea on ordinary exertion, positive neck vein distension, negative/positive pleural effusion, and negative/positive radiographic cardiomegaly; 2) More patient encounters perform better than less encounters and also have a greater average number of FHFSS mentions; and 3) Different features are more predictive during specific prediction windows.

Conclusion: This study aims to bridge the gap between epidemiological research and clinical practice as it relates to a highly prevalent, serious, costly disease. In practice, providers are left with the difficult task of basing their decisions to act on the examination of a patient in the moment, not based on a sophisticated quantitative assessment of longitudinal patient data. Our findings show that a smaller number of FHFSS can be used individually to predict early onset of heart failure.

P5.05

Assessing the Association Between Exercise Status and Poor Glycemic Control

David Mosen, ${ }^{1}$ Harry Glauber, ${ }^{1}$ Ashley Stoneburner, ${ }^{1}$ Adrianne Feldstein, ${ }^{2}$ Steve Fortmann ${ }^{1}$ 
${ }^{1}$ Kaiser Permanente Center for Health Research, Portland, OR; ${ }^{2}$ Quality Management and Systems, Kaiser Permanente Northwest, Portland, OR

Background: Increased physical activity may be associated with greater glycemic control among adults with diabetes mellitus. However, the area is understudied. The objective of the study was to examine the independent association of exercise status with poor glycemic control, adjusting for patient-level covariates.

Methods: We studied a population of Kaiser Permanente Northwest members with type 2 diabetes mellitus who were in: 1) good glycemic control (hemoglobin A1c [HbA1c]: $<8 \% ; n=15,891$ ), or 2 ) poor glycemic control (HbA1c: $>9 \%$; $\mathrm{n}=3,709)$. Additional inclusion criteria included an HbA1c test between July 1, 2014, and June 30, 2015, age 18-plus at time of test, and continuous health plan coverage 12 months prior to HbA1c test. The primary independent was current physical activity status - whether an individual exercised 4 or more times per week (yes vs no) — and was assessed as closely as possible to the HbAlc test date. Multiple logistic regression was used to analyze the independent association of exercise status with poor glycemic control, adjusting for demographics, medication adherence, medical comorbidities, health care utilization, receipt of diabetes mellitus care management services and intensity of diabetes mellitus treatments.

Results: Those who exercised 4 or more times per week were less likely to have poor glycemic control (odds ratio: 0.75; 95\% confidence interval: $0.68-0.82 ; \mathrm{P}<0.0001)$ compared with those who exercised 3 or fewer times per week.

Conclusion: Increased exercise is independently associated with a lower likelihood of poor glycemic control among an adult population with type 2 diabetes mellitus. Because physical activity is a potentially modifiable factor, further studies are needed to evaluate whether interventions aimed at increasing physical activity result in subsequent gains in glycemic control.

\section{P5.06}

\section{Loop Diuretic Use in the Months and Years Preceding a Heart Failure Diagnosis: A Case- Control Study}

David Knorek, ${ }^{1}$ Steven Steinhubl, ${ }^{2}$ Christopher deFilippi, ${ }^{3}$ Kenney $\mathrm{Ng},{ }^{4}$ Roy Byrd, ${ }^{4}$ Zahar Daar, ${ }^{5}$ Walter Stewart ${ }^{6}$

${ }^{1}$ Geisinger Health System, Danville, PA; ${ }^{2}$ Scripps Translational Science Institute, La Jolla, CA; ${ }^{3}$ Inova Heart and Vascular Institute, Falls Church, VA; ${ }^{4} I B M$ Research, Cambridge, MA; ${ }^{5}$ Geisinger Health System, Danville, PA; ${ }^{6}$ Sutter Health, Walnut Creek, CA

Background: Loop diuretics are routinely prescribed for patients with heart failure (HF) to minimize symptoms due to fluid overload, but relatively little is known about prescribing patterns in individuals preceding a formal HF diagnosis. We want to understand the prescribing of loop diuretics in primary care patients without a HF diagnosis and to determine if their use signals an increased probability of a future HF diagnosis.

Methods: A nested case-control study of 1,288 new-onset (incident) $\mathrm{HF}$ cases and 10,319 matched controls was completed with electronic health record data extracted between 2001 and 2010 from primary care practices affiliated with the Geisinger Health System. New loop diuretic prescriptions, and associated orders $<36$ months before and 12 months after an incident HF diagnosis in cases and a comparable time in controls, were evaluated.

Results: Loop diuretic use was significantly greater $(11.3 \%$ vs $3.5 \%, \mathrm{P}<0.001$ ) in future HF cases compared with controls 2 to 3 years preceding a HF diagnosis. Their use progressively increased in cases such that by 3 to 5 months preceding a HF diagnosis, $27.1 \%$ of cases versus $7.5 \%$ of controls $(\mathrm{P}<0.001)$ had been prescribed a loop diuretic. Edema (ICD-9 code 782.3) and essential hypertension (ICD-9 code 401.x) were the most commonly associated diagnoses for starting a loop diuretic in both eventual HF cases as well as in controls. By multivariate analysis, being prescribed a loop diuretic was one of the strongest clinical predictors of an eventual diagnosis of HF (odds ratio: 2.44, 95\% confidence interval: $2.10-2.83, \mathrm{P}<0.001)$.

Conclusion: In primary care, loop diuretic use is not uncommon in the months and years preceding a formal incident HF diagnosis. These results suggest there is substantial opportunity to improve the diagnosis and earlier treatment of individuals with HF.

\section{P5.07}

\section{Patterns of Prednisone Use During Pregnancy: Daily and Cumulative Dose}

Kristin Palmsten, ${ }^{1}$ Matthieu Rolland, ${ }^{1}$ Mary F. Hebert, ${ }^{2}$ Megan E.B. Clowse, ${ }^{3}$ Michael Schatz,, ${ }^{4}$ Ronghui Xu, ${ }^{1}$ Christina D. Chambers ${ }^{1}$

${ }^{1}$ University of California, San Diego, La Jolla, CA; ${ }^{2}$ University of Washington, Seattle, WA; ${ }^{3}$ Duke University Medical Center, Durham, NC; ${ }^{4}$ Kaiser Permanente Medical Center, San Diego, CA

Background: Descriptions of oral corticosteroid use during pregnancy are usually limited to trimester prevalence estimates. We sought to better characterize the use during pregnancy.

Methods: Data were collected from MotherToBaby Pregnancy Studies (2005-2014) that enrolled pregnant women with asthma and autoimmune diseases before gestational week 20. Information on medication use and pregnancy outcomes was collected by telephone interview at enrollment, 24 and 32 weeks gestation, and after delivery, 
plus by medical record review. Women were included if they had a live or still birth and reported dates of oral prednisone use and dose during pregnancy. Women were classified by diagnosis: asthma $(n=15)$, rheumatoid arthritis $(n=254)$, Crohn's disease $(n=39)$, ankylosing spondylitis $(n=15)$, and $>1$ autoimmune disease $(n=29)$. Prednisone daily dose and cumulative dose by gestational day were plotted using a heatmap for each individual and stratified by disease. To summarize the many observations for rheumatoid arthritis, we used k-means clustering method to identify and plot group trajectories for prednisone dose. The associations between trajectory group and maternal age, race/ethnicity, socioeconomic status, obesity, rheumatoid arthritis severity as measured by the Health Assessment QuestionnaireDisability Index (HAQ) (range: 0-3) before gestational week 20, and gestational age at delivery were evaluated.

Results: Women used prednisone on 1 to 292 days total during pregnancy. Daily doses ranged from 2 to $60 \mathrm{mg}$ for asthma; $<1$ to $60 \mathrm{mg}$ for rheumatoid arthritis, ankylosing spondylitis and $>1$ autoimmune disease; and $<1$ to $70 \mathrm{mg}$ for Crohn's disease. Total cumulative dose ranged from 15 to $1,325 \mathrm{mg}$ for asthma, 8 to $6,225 \mathrm{mg}$ for rheumatoid arthritis, 10 to $9,105 \mathrm{mg}$ for Crohn's disease, 60 to 2,720 $\mathrm{mg}$ for ankylosing spondylitis, and 20 to $5,120 \mathrm{mg}$ for $>$ 1 autoimmune disease. High-dose prednisone for a short duration was more common for asthma, whereas lower doses for longer was more common for rheumatoid arthritis. For women with rheumatoid arthritis, the highest versus lowest cumulative dose trajectory groups had significantly different disease severity (median HAQ: 0.7 vs 0.0 ) and gestational length (gestational weeks: 35.9 vs 39.0 ).

Conclusion: The individual-level plots illustrate variability in prednisone dosage (amount and pattern), which may impact pregnancy outcomes. Group trajectories, used to summarize these factors, provide an alternative to the typical trimester exposure approach when studying risks of corticosteroids and other medications during pregnancy.

\section{P5.08}

Patient Experience-of-Care Is Associated With Adherence/Persistence to Cardiometabolic Disease Medications in an Ambulatory Setting

Robert Romanelli, ${ }^{1}$ Vani Nimbal, ${ }^{1}$ Qiwen Huang, ${ }^{1}$ Wang Xiang, ${ }^{1}$ Joyce LaMori, ${ }^{2}$ Dilesh Doshi, ${ }^{2}$ Sukyung Chung ${ }^{1}$

${ }^{1}$ Palo Alto Medical Foundation Research Institute, Palo Alto, CA; ${ }^{2}$ Janssen Scientific Affairs, Population Health Research, Titusville, $\mathrm{NJ}$

Background: We aimed to investigate associations between patient ratings of experience-of-care and medication adherence or persistence to cardiometabolic disease (CMD) medications in an ambulatory care setting.

Methods: This retrospective study was conducted using electronic health records and administrative data from an ambulatory care network in Northern California. We included managed-care beneficiaries ( $\geq 18$ years) with a prescription for a CMD medication between 2010 and 2014. CMD medications included antihyperglycemic, antihyperlipidemic and antihypertensive agents. Within each class, we chose the first medication prescribed within 14 days of an office encounter that was associated with a completed Press-Ganey patient-experience survey. Primary outcome measures were adherence (proportion of days covered $\geq 0.80$ ) and persistence (no gap in pharmacy fills $>$ $1.5 \times$ of a day's supply). The main predictor variables were experience-of-care domains, which comprised health care providers' (a) explanation about problems or conditions; (b) effort to include patients in treatment decisions; (c) information about medications; and (d) information about follow-up care. Experience-of-care was rated on a scale of 1 (very poor) to 5 (very good). Multivariable logistic regression models were fitted to assess associations between adherence or persistence (yes/no) and ratings for each experience-of-care domain (dichotomized as 5 vs $<5$ ), with statistical adjustment for important patient, prescription and provider characteristics. Odds ratios (ORs) were generated.

Results: Eligibility criteria were met by 5,045 patients. The majority of patients were $\geq 65$ years old $(61.8 \%)$; median of CMD medications was 2. Patients receiving antihyperglycemic medications had higher odds of adherence when they had better experience-of-care with "inclusion in treatment decisions" (OR: 1.64) and "information about follow-up care" (OR: 1.60). Patients receiving antihyperlipidemic medications had higher odds of persistence when they had better experiences-of-care within each domain (range of ORs: 1.38-1.57). Patients receiving antihypertensive medications had higher odds of persistence when they had better experiences-of-care with "inclusion in treatment decisions" (OR: 1.52) and "information about medications" (OR: 1.55).

Conclusion: In an ambulatory care setting, better experienceof-care with a health care provider in making shared treatment decisions was consistently a positive predictor of adherence/persistence. The relationship between other domains of experience-of-care and adherence/persistence differed by therapeutic class. Improving adherence or persistence to CMD medications through better patient experiences with their health care providers will likely require tailored approaches.

P5.09

A Shared Medical Appointment on the Benefits and Risks of Opioids in Chronic Pain Management: Evaluation of a Pilot Program in an Ambulatory Setting

Robert Romanelli, ${ }^{1}$ Marina Dolginsky, ${ }^{2}$ Yuliya Byakina, ${ }^{3}$ Deborah Bronstein, ${ }^{2}$ Sandra Wilson ${ }^{1}$ 
${ }^{1}$ Palo Alto Medical Foundation Research Institute, Palo Alto, CA; ${ }^{2}$ Palo Alto Medical Foundation, Palo Alto, CA; ${ }^{3}$ University of California, San Francisco, San Francisco, CA

Background: We sought to evaluate a pilot shared medical appointment (SMA) designed to educate patients on the safe use of opioids and build empowerment in self-managing chronic pain (CP).

Methods: This prospective study was conducted at an ambulatory clinic within a health care system in Northern California. The pilot SMA began in February 2016. The SMA is a single 90-minute billable office appointment led by a primary care physician to a group of 15-20 patients. Patients were included in the study if they were adults with a CP condition and referred to the SMA by a clinic physician. Data were collected by a brief survey administered to patients immediately before and immediately after the SMA. In addition to demographics and clinical characteristics, the survey collected information on four patient-experience domains: 1) understanding of $\mathrm{CP}$; 2) confidence in $\mathrm{CP}$ selfmanagement; 3 ) confidence in health care providers' ability to help manage $\mathrm{CP}$; and 4) satisfaction with care received within the health care system for CP. Domains were measured on a scale of 0 (worst) to 5 (best). Mean differences in prepost ratings were assessed by a paired t-test; P-value $<0.05$ was considered statistically significant.

Results: Between February and August 2016, 130 patients attended the SMA and completed the survey. The majority of patients were $\geq 50$ years of age $(69.6 \%)$, and $56.2 \%$ were female. The most prevalent CP condition was back/neck pain (73.8\%), followed by joint/bone pain $(53.6 \%) ; 63.8 \%$ of patients reported $>1 \mathrm{CP}$ condition. In a comparison of pre-post responses, patients showed statistically significant increases in confidence in self-managing CP $(+0.44$; $95 \%$ confidence interval [CI]: $0.29-0.59 ; \mathrm{P}<0.001)$ and in their health care providers' ability to help manage CP $(+0.28 ; 95 \% \mathrm{CI}$ : $0.14-0.43$; $\mathrm{P}<0.001)$. No changes were observed in patents' understanding of, or satisfaction with care received for, $\mathrm{CP}$.

Conclusion: In an ambulatory care setting among patients with various $\mathrm{CP}$ conditions, an SMA targeting the risk and benefits of opioids was associated with prompt improvements in patients' confidence in self-managing CP and in their health care providers' ability to help manage CP. Such confidence can lay the foundation for increased patient engagement and activation in pain management.

P5.10

Comparative Effectiveness of Clinical Intensive Behavior Therapy for Obese Adults

Jeffrey VanWormer, ${ }^{1}$ Tenisha Hill, ${ }^{1}$ Brian Hoch ${ }^{1}$

${ }^{1}$ Marshfield Clinic Research Foundation, Marshfield, WI

Background: About 1 in 3 adults are obese in the United States, but the delivery of clinical weight loss counseling is infrequent. The Centers for Medicare \& Medicaid Services began reimbursing for intensive behavior therapy (IBT) for obese adult beneficiaries in late 2011, but the real-world effectiveness of IBT has not been rigorously evaluated. This study compared weight change over 1 year between obese adult patients who did and did not receive IBT.

Methods: A retrospective cohort of obese, adult virtual data warehouse members from the Marshfield Clinic Health System was assembled. Two groups were compared: adult patients who had $\geq 1$ or more IBT visits versus those who had no IBT visits. The comparison group was matched on body mass index (BMI), age, sex and health care coverage. The primary outcome was body weight over 1 year. Weight loss surgery patients were excluded.

Results: There were 107 patients exposed to IBT ( $\sim 1 \%$ of those eligible) and 320 in the matched unexposed group. Patients who received IBT were $79 \%$ female and $72 \%$ elderly, with a mean (standard deviation) baseline BMI of $39.8(7.4) \mathrm{kg} / \mathrm{m}^{2}$. IBT patients completed a median of 5 visits over 1 year, with visits being led predominantly by registered dietitians. Visit attendance dropped quickly though, with just $1 \%$ of IBT patients completing all 22 allotted visits. Body weight in the matched comparison group remained stable over 1 year, whereas IBT patients lost 3.9 (8.2) kg $(\mathrm{P}<0.001)$.

Conclusion: Clinic-based IBT was moderately effective, as participants lost about $4 \%$ of their baseline weight over 1 year, or about 1 additional BMI unit. As expected, those who did not participate in IBT did not lose weight. But program participation and retention was quite low, as the relatively few patients exposed to IBT also completed very few of their allowable IBT visits, even after accounting for 6-month restrictions on program eligibility. To impact population level obesity, more research is needed on how to attract and engage obese adults in clinical weight management programs.

P5.12

\section{What Factors Facilitate Weight Loss Among Medicaid Beneficiaries Participating in the Diabetes Prevention Program?}

Gabriela Vazquez-Benitez, ${ }^{1}$ Jay Desai, ${ }^{1}$ Gretchen Taylor, ${ }^{2}$ Sara Vine, ${ }^{3}$ Julie Anderson, ${ }^{1}$ Joyce Garrett, ${ }^{4}$ Todd Gilmer, ${ }^{5}$ Houa Vue-Her, ${ }^{2}$ Jeff Schiff, ${ }^{4}$ Sarah Rinn, ${ }^{4}$ Katelyn Engel, ${ }^{2}$ Amy Michael, ${ }^{2}$ Mary Becker, ${ }^{1}$ Patrick $\mathrm{O}^{\prime}$ Connor ${ }^{1}$

${ }^{1}$ HealthPartners Institute, Bloomington, MN; ${ }^{2}$ Minnesota Department of Health, St. Paul, MN; ${ }^{3}$ Hennepin County Medical Center, Minneapolis, MN; ${ }^{4}$ Minnesota Department of Human Services, St. Paul, MN; ${ }^{5}$ University of California, San Diego, La Jolla, CA

Background: The implementation of the Diabetes Prevention Program (DPP) with culturally diverse and low- 
income participants has had varied success. The We Can Prevent Diabetes study was a pragmatic three-arm clusterrandomized trial with Medicaid beneficiaries to evaluate the effectiveness of financial incentives on group-delivered DPP participation and weight loss. The purpose of this analysis is to identify participant and programmatic factors associated with $5 \%$ weight loss.

Methods: Among Medicaid participants attending at least 4 of the 16 DPP core sessions, we examined factors associated with 5\% weight loss at any time during the DPP core session period or a sustained 5\% weight loss over the 16 sessions. The latter was determined using a latent class trajectory analysis. Univariate associations with $\mathrm{P}<0.2$ were included in multivariable logistic models.

Results: Among the 658 Medicaid beneficiaries in our analysis, $23 \%$ met each of the two outcome measures, $62 \%$ were 45-64 years old, 72\% were women, 82\% were not white, 26\% were non-English speakers (Spanish, Somali, Hmong), 76\% were obese, 18\% received DPP led by a community member as a lifestyle coach, and 68\% were in a financial incentive intervention arm. After adjustment, attending 9 or more sessions (odds ratio: 5.7, 95\% confidence interval: $2.6-12.7)$, reporting physical activity 9 or more times $(2.9,1.4-5.9)$ and being a non-English speaker $(2.0,1.2-3.7)$ were associated with achieving 5\% weight loss at any time. Only the latter was associated with a sustained 5\% weight loss $(2.1,1.3-3.6)$. After adjustment, allocation to a financial incentive arm was not associated with either of the $5 \%$ weight-loss metrics.

Conclusion: Session attendance and physical activity reporting may improve successful weight loss during the DPP. Non-English speaking participants had the most success in achieving a sustained $5 \%$ weight loss. This may be due to tailoring the DPP curriculum to the specific culture, and using members of the community as lifestyle coaches.

\section{P05.13}

Long-Term Outcomes of a Cluster-Randomized Trial Testing the Effects of Blood Pressure Telemonitoring and Pharmacist Management

Stephen Asche, ${ }^{1}$ Anna Bergdall, ${ }^{1}$ Steven Dehmer, ${ }^{1}$ Beverly Green, ${ }^{2}$ JoAnn Sperl-Hillen, ${ }^{1}$ Patrick O'Connor, ${ }^{1}$ Rachel Nyboer, ${ }^{1}$ Pamala A. Pawloski, ${ }^{1}$ Michael Maciosek, ${ }^{1}$ Nicole Trower, ${ }^{1}$ Karen Margolis ${ }^{1}$

${ }^{1}$ HealthPartners Institute, Minneapolis, $M N ;{ }^{2}$ Group Health Research Institute, Seattle, WA

Background: Hypertension is a common condition and leading cause of cardiovascular disease. We previously reported results of a cluster-randomized trial evaluating a home blood pressure telemonitoring and pharmacist management intervention, with significant reductions in systolic blood pressure (SBP) favoring the intervention arm found over 6,12 and 18 months and in diastolic blood pressure (DBP) found over 6 and 12 months. This analysis examined the durability of the intervention effect on blood pressure through 54 months of follow-up.

Methods: The Hyperlink trial randomized 16 primary care clinics having 450 study-enrolled patients with uncontrolled hypertension to either telemonitoring intervention (TI) or usual care (UC) study arms. Blood pressure was measured as the mean of three measurements obtained at each research clinic visit. General linear mixed models utilizing a direct likelihood-based ignorable approach for missing data were used to examine change from baseline to 54 months in SBP and DBP.

Results: Blood pressure measurements were obtained from $164(74 \%)$ in UC and $162(71 \%)$ of TI patients at the 54-month follow-up visit. For TI patients, baseline SBP was $148.2 \mathrm{~mm} \mathrm{Hg}$ and 54-month follow-up was $131.2 \mathrm{~mm} \mathrm{Hg}$ $(-17.0 \mathrm{~mm} \mathrm{Hg}, \mathrm{P}<0.001)$. For UC patients, baseline SBP was $147.7 \mathrm{~mm} \mathrm{Hg}$ and 54-month follow-up was $131.7 \mathrm{~mm}$ $\mathrm{Hg}(-16.0 \mathrm{~mm} \mathrm{Hg}, \mathrm{P}<0.001)$. The differential reduction by study arm in SBP from baseline to 54 months was - $1.0(95 \%$ confidence interval $[\mathrm{CI}]:-5.4$ to $3.4, \mathrm{P}=0.63)$. For TI patients, baseline DBP was $84.4 \mathrm{~mm} \mathrm{Hg}$ and 54-month follow-up was 77.8 (-6.6 mm Hg, $\mathrm{P}<0.001)$. For UC patients, baseline DBP was $85.1 \mathrm{~mm} \mathrm{Hg}$ and 54-month follow-up was $79.1 \mathrm{~mm} \mathrm{Hg}$ (-6.0 mm Hg, P<0.001). The differential reduction by study arm in DBP from baseline to 54 months was $-0.6 \mathrm{~mm} \mathrm{Hg}$ (95\% CI: -3.5 to $2.4, \mathrm{P}=0.67$ ).

Conclusion: Significant blood pressure reductions in the TI arm relative to UC were no longer seen at 54-month follow-up. More work is needed to ascertain the optimal duration and reinforcement that could be used to maintain intervention benefits over a longer period of time.

\section{COMMUNICATIONS/KNOWLEDGE ACQUISITION AND TRANSMISSION}

P6.01

Suspected Underdiagnosis of Respiratory Syncytial Virus in a Large Health System: Early Findings From Electronic Health Record Data Exploration and Conversations With a Hospitalist

Jessica Liu, ${ }^{1}$ Heather Law, ${ }^{1}$ Sarah Robinson, ${ }^{1}$ Richard Liu, ${ }^{2}$ Lisa Dean, ${ }^{1}$ Alice Pressman ${ }^{1}$

${ }^{1}$ Research, Development and Dissemination, Sutter Health, Walnut Creek, CA; ${ }^{2}$ Mills-Peninsula Medical Hospital, Sutter Health, Burlingame, CA

Background: Respiratory syncytial virus (RSV) is a common virus that infects the lungs and breathing passages. Although it manifests as mild cold-like symptoms in healthy adults and children, RSV can be life-threatening among infants, the elderly and immunocompromised adults. We set out to describe the adult-patient experience with RSV, 
examining rates of diagnosis within a large health care system, but quickly learned that the project could not be completed without one stakeholder's (a hospitalist) experience with RSV.

Methods: We conducted a retrospective cohort study of hospitalized adults (age: 18+ years) with a diagnosis of RSV within a large health system from October 1, 2013, to June 1, 2016. RSV was identified by evidence of one or more diagnosis codes (ICD-9: 079.6, 466.11 and 480.1; ICD-10: B97.4, J12.1 and J21.0) and/or positive laboratory findings for panels including RSV.

Results: We identified 72 cases of diagnosed RSV across 24 Sutter Health hospitals during the study period. RSV patients averaged 74 years (standard deviation: 18.6); 47 (65\%) were female; 47 (65\%) were white, 10 (14\%) were Asian, and the remaining 15 (21\%) were other minority. Cases of RSV were disproportionate among hospitals across the system; one hospital accounted for 55 (76\%) cases. A hospitalist at this site confirmed that in 2014, this facility began ordering a new respiratory viral panel offered only through the local public health department. This test, not available at other hospitals, screens for influenza and other respiratory infection including RSV.

Conclusion: The clinical features of RSV may be difficult to distinguish from influenza and bacterial respiratory infections. Although there are currently no specific treatments available for RSV, it is important to distinguish between viral and bacterial origins to minimize inappropriate use of antibiotics. The disparate counts of RSV at the single hospital allowed us to recognize underdiagnosis in our system. We began our study with the objective to describe the RSV patient journey, but adapted to describe its patterns of diagnosis in a multihospital health system, including a pre-post implementation of respiratory viral panel testing analysis. Our study highlights the impact of engaging subject matter experts as key stakeholders early in the process.

\section{P6.02}

Exploring Prevalence of Discussions of e-Cigarettes Use During Tobacco Cessation Counseling and Smokers' Understanding of e-Cigarette Use

Andy S.L. Tan, ${ }^{1}$ Gwen L. Alexander, ${ }^{2}$ Kathleen Mazor, ${ }^{3}$ Amanda Holm, ${ }^{2}$ Kasisomayajula Viswanath ${ }^{1}$

${ }^{1}$ Dana-Farber Cancer Institute, Boston, MA; ${ }^{2}$ Department of Public Health Sciences, Henry Ford Health System, Detroit, MI; ${ }^{3}$ Meyers Primary Care Institute, University of Massachusetts Medical School, Worcester, MA

Background: E-cigarettes are increasingly popular among smokers who are using these products to reduce or quit smoking cigarettes completely. The purpose of this study is to explore the prevalence of discussions during tobacco cessation counseling at a Midwestern integrated health system's tobacco intervention program (TIP), topics discussed, and smokers' understanding of e-cigarettes use for cessation.

Methods: Two tobacco treatment counselors recorded logs of e-cigarette discussions, which occurred during cessation counseling calls, between May and August 2016. We conducted phone interviews with a purposive sample of 17 smokers (age range: 29 to 70 years; sex: 16 female; race: 6 African American, 4 white, 7 race not indicated) within 3 days of counseling sessions. Of the 17, 11 participants had not discussed e-cigarettes and 6 had discussed e-cigarettes with their counselors. We analyzed interview transcripts and summarized topics discussed, information sources, information needs and opinions about e-cigarette use for cessation.

Results: Among 460 counseling sessions, 24 (5\%) included discussions about e-cigarettes. Ten of these 24 sessions (42\%) occurred during initial enrollment and the remainder occurred during follow-up counseling sessions. All discussions about e-cigarettes were initiated by smokers. Of the 6 smokers who discussed e-cigarettes, the topics discussed were current and previous e-cigarette use, side effects, lack of evidence of safety and effectiveness, addictiveness and counselor's recommendation against using e-cigarettes. Sources of information about e-cigarettes included television, convenience stores or gas stations, word-of-mouth, news/magazines/online articles and smoke shops. Participants expressed information needs including side effects, efficacy to help quit smoking and how to use e-cigarettes. They also perceived advantages (more socially acceptable, lack of secondhand smoke) and disadvantages of e-cigarette use (cost, not helpful to quit smoking, potential health risks and other dangers including explosions, addictiveness).

Conclusion: Discussion about e-cigarettes was not common among smokers and tobacco treatment counselors. Smokers undergoing cessation counseling expressed confusion and need for information about e-cigarette use, generally, and the use of e-cigarettes for quitting smoking. Further research is needed to develop tools to improve patient-provider discussions about cessation and e-cigarette use and to increase patients' knowledge about e-cigarette use.

P6.03

\section{Evolving Dashboards: Honing Metrics by Engaging} Stakeholders

Sarah Robinson, ${ }^{1}$ Theresa Schrider, ${ }^{1}$ Julie Anne Miller, ${ }^{1}$ Anjali Franco, ${ }^{1}$ Nicole Oehmke, ${ }^{1}$ Kristen Azar, ${ }^{1}$ Alice Pressman ${ }^{1}$

${ }^{1}$ Sutter Health, Walnut Creek, CA

Background: Project dashboards provide necessary insight to the impact and value of patient-centered programs in a health care system. When aptly constructed, administration can utilize the dashboard to create a positive feedback loop 
that enables rapid learning and adaptation. Challenges in creating and modifying a dashboard are common, particularly when information and resources are limited. This can lead to misinterpretation of data and staff frustration. We established a partnership between Sutter Health's Research, Development and Dissemination department and the case managers at a two-campus hospital in Alameda County, California, to reconstruct their organizational dashboard. We describe the process and lessons learned.

Methods: The original dashboard was framed into four sections, three describing specific initiatives and one addressing secondary outcomes. To evolve the dashboard, we used an iterative and heuristic process, convening several meetings focused on methodically discussing the value of each section and metric. During these conversations, we engaged the stakeholders by soliciting the function of each initiative as well as the merit of each metric. After discussing which metrics were vital, we created a visual report showing trends in the identified data elements. We then presented the new dashboard to a group of stakeholders and elicited feedback.

Results: Preserving much of the earlier framework, modifications were primarily focused on the metrics. We removed all target values and deprecated metrics that were no longer relevant to the program. Some measurements were redefined to more accurately represent programimpact goals while other metrics remained unchanged. Feedback was generally positive and produced productive dialogues concerning program aims and future states.

Conclusion: By taking the time to understand the objectives of the program as well as the strategy, we produced a dashboard that enabled our colleagues to make organizational decisions and identify program successes and weaknesses. The evaluation of each metric ensured goal-alignment and justification, thereby eliminating confusion about program intentions and limitations. Additionally, visuals helped focus conversations around key aspects and outcomes, thereby enabling stakeholders to communicate advancements in a cogent manner. Though they require time and flexibility, dashboards are instrumental in reporting on the health of system initiatives.

\section{DELIVERY SYSTEM COLLABORATIONS}

\section{P7.01

Patient-Provider Communication During
Posttreatment Breast Cancer Care: Findings From
a Kaiser Permanente Northern California Pilot
Project

Neetu Chawla, ${ }^{1}$ Michael Sanchez, ${ }^{1}$ Jayson Harpster, ${ }^{1}$ Leslie Mance Brenman, ${ }^{1}$ Raymond Liu ${ }^{1}$

${ }^{1}$ Kaiser Permanente Northern California, Oakland, CA
Background: Patient-provider communication is essential to delivering high-quality cancer care, including posttreatment when survivors have many complex care needs. In this study, we used data collected for quality improvement from a small feasibility sample to examine patient perceptions of provider communication and inform the development of a new Oncology Survivorship Clinic model.

Methods: As part of a pilot project conducted at Kaiser Permanente Northern California, we surveyed 51 breast cancer patients posttreatment. The survey included a communication measure from the 2011 Medical Expenditure Panel Survey: Experiences with Cancer Survivorship Supplement evaluating provider discussions of: 1) surveillance for recurrence, 2) late or long-term treatment effects, 3) healthy lifestyle behaviors, and 4) emotional or social needs. We also examined reports of the 6 core functions of patient-centered communication (ie, managing uncertainty, responding to emotions, making decisions, fostering healing relationships, enabling selfmanagement, exchanging information) using a measure from the Health Information National Trends Survey 4: Cycle 4. Part of the purpose was to evaluate acceptability of a new Oncology Survivorship Clinic utilizing nonphysician providers.

Results: The sample included 51 breast cancer patients surveyed in 2016 within 6 months of treatment completion. All women were stage $0-3$. Overall, sizable proportions received detailed communication about surveillance $(65 \%)$, treatment side effects (46\%), emotional needs $(41 \%)$ and healthy lifestyles $(71 \%)$, and the majority received patientcentered communication (range: $60 \%-73 \%$ based on core function). Particular gaps were noted related to provider communication about treatment side effects (54\%), emotional/social needs (59\%), managing uncertainty (35\%) and responding to emotions (40\%).

Conclusion: Our very preliminary findings suggest that the majority of women had positive communication experiences, including with nonphysician providers. However, clear communications gaps existed, underscoring future avenues for research and care delivery interventions to address the needs of breast cancer patients more comprehensively.

P7.02

Retrieving Medical Records Within FDA's Sentinel Distributed Network: Lessons Learned During a Protocol-Based Assessment Involving 13 Data Partners

Crystal Garcia, ${ }^{1}$ Candace Fuller, ${ }^{1}$ Madelyn Pimentel, ${ }^{1}$ Eric Ammann, ${ }^{2}$ Elizabeth Chrischilles, ${ }^{2}$ Scott Winiecki ${ }^{3}$

${ }^{1}$ Harvard Medical School and Harvard Pilgrim Health Care Institute, Boston, MA; ${ }^{2}$ University of Iowa College of Public Health, Iowa City, IA, ${ }^{3}$ U.S. Food and Drug Administration, Silver Spring, $M D$ 
Background: The U.S. Food and Drug Administration's Sentinel system has developed the capability to conduct active safety surveillance of marketed medical products in a large distributed network. Medical record retrieval in such an environment is logistically challenging and resourceintensive. We describe lessons learned during a protocolbased assessment of thromboembolic events (TEE) following intravenous immunoglobulin (IGIV) administration, a project that necessitated chart retrieval requests for a large number of patients from 13 Sentinel data partners.

Methods: The Sentinel IGIV-TEE workgroup requested medical records pertaining to 442 potential post-IGIV-TEE cases identified from the Sentinel Distributed Database. Charts were requested from 13 different data partners (including 4 Health Care Systems Research Network members). Six partners were claims-based health plans (CBHPs), and 7 were integrated care delivery systems (ICDSs). Dates of medical records spanned March 2006 to February 2013. Records were received and tracked by the Sentinel Operations Center at Harvard Pilgrim Health Care Institute. We report on the retrieval process, chart retrieval rates and reasons identified by data partners for nonretrieval by data partner type.

Results: The vast majority of cases identified for record retrieval were from CBHPs (88\%). Retrieval processes varied by data partner type: 4 of the 7 CBHPs contracted with vendors to conduct record retrieval across their facilities, whereas the ICDSs relied on internal personnel to either electronically pull charts or seek paper records. These differing processes had implications for troubleshooting missing records and information, resulting in diverse strategies for data partner-facility consultation. Rates of retrieval varied by data partner type (64\% in CBHPs vs $94 \%$ in ICDSs) as did speed of retrieval and resources. Reasons records were unobtainable fell into three major categories: inability to map Sentinel Distributed Database records to patient and provider identifiers needed for retrieval; provider refusal to participate due to legal/ compliance/HIPAA concerns; and missing information (eg, no record of patient at facility, charts for the requested service dates were unavailable).

Conclusion: We will describe key lessons learned, broadly applicable to any chart retrieval project in a distributed environment, and include a discussion focused on the pros and cons of working with multiple collaborators with different data environments.

\section{P7.03}

Leveraging the Virtual Data Warehouse to Support Implementation of Precision Medicine for Oncology at Catholic Health Initiatives

John Weeks, ${ }^{1}$ Jan Lowery, ${ }^{1}$ David Bailey, ${ }^{1}$ Elizabeth Evans, ${ }^{1}$ Penne Perry, ${ }^{1}$ Lela McFarland ${ }^{1}$

${ }^{1}$ Catholic Health Initiatives, Englewood, $\mathrm{CO}$
Background: Catholic Health Initiatives (CHI) is implementing a pilot program for precision medicine (PM) in oncology. The application to support this program integrates patients' demographic, clinical, treatment and outcome data with molecular data from tumor testing into a central data store, which is used to inform personalized treatment options and match individuals with ongoing clinical trials. We assessed the extent to which the virtual data warehouse (VDW) could support the data needed for this initiative.

Methods: The CHI Institute for Research and Innovation data science team reviewed data requirements for the application with respect to content and format, and compared these to current VDW specifications. We identified data gaps between the application requirements and the VDW. We also identified data gaps within our own organization to support both of these and explored how we might leverage our partnership with the operations group through this initiative to facilitate acquisition of these data for mutual benefit.

Results: Of the 70 required data elements, 42 elements could be mapped to the VDW specifications (60\%). We identified data gaps for patient observations such as functional performance scores, drug orders and administration, and narratives for procedures performed, which the VDW does not capture. The VDW also does not contain information on medications dispensed in inpatient settings. Significant gaps in data currently available at CHI to support the VDW and the PM application exist with pharmacy (medications) and infusion data. Results from this gap analysis have informed ongoing discussions with the PM operations team about the feasibility of acquiring these data, which would support both applications, and providing the funding through the PM initiative to do so. Further, we are discussing options for acquiring genomics data generated by PM into our data repository to be available for future research.

Conclusion: Our analysis suggests that the VDW can largely support the PM initiative, which would facilitate broad and efficient implementation of PM across multiple facilities. Data gaps that we identified inform potential need and opportunities for expanding the VDW to support PM initiatives. This effort highlights an example of a bidirectional partnership between research and health care operations, which is an essential component for learning health care systems.

P7.04

Validation of a Prediction Model Used at Kaiser Permanente Northwest in Collaboration With Operations

Ning Smith, ${ }^{1}$ Xiuhai Yang, ${ }^{1}$ Amanda Petrik, ${ }^{1}$ Yvonne Rice, ${ }^{2}$ Keith Crowland, ${ }^{2}$ Delilah Moore,${ }^{2}$ Eric Johnson ${ }^{1}$

${ }^{1}$ Kaiser Permanente Center for Health Research, Portland, OR; ${ }^{2}$ Kaiser Permanente Northwest, Portland, OR 
Background: The LACE risk score is used by Kaiser Permanente Northwest (KPNW) to identify patients at high risk for hospital readmission or mortality. LACE triggers the delivery of Transitions of Care interventions to reduce unnecessary readmissions. However, LACE was put into practice before it was validated for Kaiser Permanente hospitals. This study validated the LACE model in the KPNW patient population using routine electronic medical record data (not research data from the virtual data warehouse) to evaluate the accuracy and applicability of the predictions. The validation was only possible through collaboration with KPNW operations.

Methods: This is a retrospective study of adult patients hospitalized from August 2014 to July 2015 at Sunnyside Medical Center (KSMC) at KPNW. Only the initial admission was included. The study outcome was readmission to the hospital or death within 30 days of discharge. The C-statistic was compared to the published value (Van Walraven et al, 2010). We assessed calibration (accuracy) graphically before and after we updated the LACE model with the KSMCspecific intercept.

Results: Of the 9,699 patients, $10.8 \%$ experienced the outcome. The C-statistic for KPNW (0.728) was slightly higher than the published value from Ontario hospitals (0.7114). The published LACE score was well calibrated with the KPNW population (observed vs predicted risks for the low $/ \mathrm{mid} /$ high-risk groups were $0.042 / 0.102 / 0.256$ vs $0.053 / 0.112 / 0.234$, respectively). The updated LACE model with the KSMC-specific intercept (ie, probability in the lowest-risk patients) resulted in slightly better calibration (predicted risks for the low/mid/high-risk groups were $0.043 / 0.106 / 0.247)$. To target high-risk patients for Transitions of Care interventions, KPNW applies a cut-off of 11 or more LACE points, which resulted in a positive predictive value of $25.6 \%$.

Conclusion: The LACE score validated successfully at KPNW's KSMC, with only a slight underestimate of risk in high-risk patients. Updating LACE improved the predicted risk modestly (1.3\%) for high-risk patients. Kaiser Permanente operations will continue to collaborate with colleagues in research to evaluate the LACE+ index and other predictors of readmission and mortality.

\section{P7.06}

\section{PreManage ED ${ }^{\mathrm{Tm}}$ Evaluation in Hospitals of Alameda County, California}

Xiangyi Xu, ${ }^{1}$ Franco Anjali, ${ }^{1}$ Kristen Azar, ${ }^{1}$ Nicole Oehmke, ${ }^{1}$ Nasiera Byrd, ${ }^{1}$ Alice Pressman ${ }^{1}$

${ }^{1}$ Sutter Health, Walnut Creek, CA

Background: In medically underserved markets, frequent utilization of the emergency department for nonurgent reasons suggests inadequate access to ambulatory care and contributes to suboptimal patient outcomes. Hospital-based interventions are challenging to implement because any single hospital has a limited view of a patient's health story. To address this issue, PreManage ED ${ }^{\mathrm{TM}}$ (PMED), a datasharing platform, has been implemented in 6 hospitals in Alameda County in Northern California - 4 Sutter facilities and 2 Alameda Health System facilities. In the initial stage of the evaluation, we characterized PMED use and assessed user acceptance.

Methods: In this study, we defined the population to be anyone with at least one hospital visit to a PMEDparticipating Sutter facility in the past 30 days, and examined patient emergency department use from May 30, 2016, to August 28, 2016, at the 6 participating hospitals. Using PMED and electronic health record (EHR) data, we recorded application usage and measured volume of high utilizers (defined as $3+$ encounters in 30 days at any of the participating facilities).

Results: During the study period, based on EHR, 47,455 patients made 49,453 encounters at the 4 Sutter Health emergency departments. PMED identified all encounters plus an additional $690(1.4 \%)$ not found in the EHR. From these patients, PMED flagged 4,633 (10\%) as high utilizers and identified $528(11 \%)$ of these as having a care guideline in place at 1 of the 6 facilities. Among the 3 physicians, 2 case managers, 1 nurse and 4 other staff members who completed the questionnaire, at least $80 \%$ responded favorably to all questions concerning usefulness, at least $70 \%$ responded favorably to questions of ease of use, and at least $70 \%$ had a favorable attitude towards PMED.

Conclusion: Data indicate that PMED is successful at alerting providers to high utilizers in the emergency department, and there is no evidence that any were missed. Further, PMED was able to flag those with a care guideline in place at any facility, thus reducing the chance that extraneous care guidelines be created. Users had high rates of acceptance, which indicates a high chance of success for implementation of interventions relying on PMED.

\section{GENETICS, GENOMICS, PRECISION MEDICINE}

P8.01

\section{Payer Decision-Making for Pharmacogenetic Tests: Preliminary Results}

Christine Lu, ${ }^{1}$ Stephanie Treadwell, ${ }^{1}$ Rachel Ceccarelli, ${ }^{1}$ Kathleen Mazor, ${ }^{2} \mathrm{Ann} \mathrm{Wu}^{1}$

${ }^{1}$ Department of Population Medicine, Harvard Pilgrim Health Care Institute/Harvard Medical School, Boston, MA; ${ }^{2}$ Meyers Primary Care Institute, University of Massachusetts Medical School, Worcester, MA

Background: Genetic tests are the fastest growing sector of medicine and medical science, yet there is a dearth of 
research on access to cancer-related pharmacogenetic tests. The objective is to explore payers' views about management strategies for pharmacogenetic tests and to describe criteria for coverage decisions, policy challenges and strategies used to overcome these challenges.

Methods: We conducted semi-structured interviews with representatives of U.S. private payers. Interviews were recorded and transcribed verbatim. Using a directed qualitative content analysis, two members of the research team performed open coding of the transcripts in an iterative process, building a provisional code book as coding progressed. Each interview transcript was independently read and coded by two members of the study team.

Results: Payers may not have established coverage policies for single gene tests, but even without a policy in place, these are generally accessible on a case-by-case basis. For coverage decision-making for pharmacogenetic tests, payers generally followed coverage decision-making processes originally established for pharmaceuticals. Some realize that the evidence requirements, which are established for pharmaceuticals, are not applicable to pharmacogenetic tests, particularly because the field is advancing rapidly. "Outcomes-based" risk sharing agreements with diagnostic companies are recognized as a possible option to collect evidence and limiting coverage. Some payers are introducing prior authorization requirements for pharmacogenetic tests to better manage utilization because an established coding system for tests is lacking. Another key challenge from payers' perspective is managing the use of and payment for gene panels. Laboratories provide different combination of genes in their panel tests, thus knowing which genes are tested is a challenge. Some payers do not pay for large gene panels.

Conclusion: Single pharmacogenetic tests are generally readily accessible. However, as we move from single gene tests to gene panels, payers have identified challenges and ways of overcoming those challenges as the field evolves.

\section{P8.02}

\section{Patient Views on the Use of Personal Health Information and Biological Samples for Biobank Research}

Katherine Gillespie, ${ }^{1}$ Harold Luft, ${ }^{1}$ Yasmin Hernandez, ${ }^{1}$ Sandra Lee, ${ }^{2}$ Mildred Cho, ${ }^{2}$ Stephanie Craft ${ }^{3}$

${ }^{1}$ Palo Alto Medical Foundation Research Institute, Palo Alto, CA; ${ }^{2}$ Stanford Center for Biomedical Ethics, Palo Alto, CA; ${ }^{3}$ Seattle Children's Research Institute, Seattle, WA

Background: Patients' level of knowledge about and acceptance of participating in health research varies. The increasing use of genetics for research and the emerging strategies of biobanking and precision medicine raise questions about the effects of patient education, patient understanding of health research and informed consent.
Whether these factors vary by patient race/ethnicity is an important but unexplored issue.

Methods: To explore these issues, multilingual videos were developed through an iterative process and were presented to patients in a community-based health care system. These videos presented topics relating to the research process, the relationship between health care and health research, consent, security and oversight. Twenty focus groups were conducted with African-American, Chinese, Hispanic, nonHispanic white and South Asian patients. Patients viewed the videos and then answered semi-structured questions in the focus group.

Results: Participants generally expressed a willingness to participate in a hypothetical biobank, and they acknowledged the benefits of this type of research. However, willingness to participate depended on their trust in the institution, potential users of the data and potential outcomes of the research.

Conclusion: Institutional oversight and governance structures can encourage trust among patients if they meet key patient expectations. While most patients did not say they needed to know about each study conducted, they wanted to know that their data will help people, either those with similar characteristics or the general public, in the future.

\section{P8.03}

Access and Reimbursement for Cancer-Related Pharmacogenetic Tests and Medications

\section{Ann Wu, ${ }^{1}$ Kathleen Mazor, ${ }^{2}$ Rachel Ceccarelli, ${ }^{1}$ Christine $\mathrm{Lu}^{1}$ \\ ${ }^{1}$ Harvard Pilgrim Health Care Institute, Boston, MA; ${ }^{2}$ Meyers Primary Care Institute, Worcester, $M A$}

Background: Genomic tests are the fastest growing sector of medicine and medical science, yet there is a dearth of research on access to cancer-related pharmacogenetic tests and medications. The objective of this study is to explore views of clinicians about access to pharmacogenetic tests using qualitative methods.

Methods: We conducted semi-structured interviews with a purposeful sample of clinicians who had prescribed medications that should be guided by pharmacogenetic testing. The purpose of the interviews was to explore knowledge of insurance cost-sharing for test, experienced or perceived barriers to access, and strategies used for managing costs. Interviews were recorded and transcribed verbatim. Using directed qualitative content analysis, two members of the research team performed iterative open coding of the transcripts. Each interview transcript was independently read and coded by two members of the study team.

Results: Ten interviews were conducted ( 8 oncologists, 1 nurse practitioner, 1 nurse). Of the 10 clinicians, 6 practiced in an academic hospital setting and 4 practiced 
in a community setting. Clinicians described logistical and insurance issues relating to ordering genetic tests and medications. They also reported that they ordered pharmacogenetic tests based on medical need with little communication about insurance with patients; they had few perceived and experienced barriers in access to tests but had limited awareness of coverage of tests. The process of ordering tests is variable. In comparison, clinicians were much more aware of financial and administrative barriers to access cancer therapies related to pharmacogenetic testing, including burdensome and lengthy insurance approval and reimbursement processes for both patients and providers and substantial out-of-pocket costs.

Conclusion: Currently, ordering pharmacogenetic tests is less complex than ordering cancer therapies, but this is likely to change in the near future as administrative barriers are introduced to manage volume. Better understanding of the implementation of pharmacogenetic tests into community and clinical settings will help inform future implementation strategies for other more complex genomic technologies to improve patient outcomes.

\section{P8.04}

\section{Precision Medicine Decision-Making Tool: Generic Cost-Effectiveness Analysis Models}

\section{Susan R. Snyder, ${ }^{1}$ Jing Hao, ${ }^{1}$ Zhi Geng, ${ }^{1}$ Marc Williams ${ }^{1}$ \\ ${ }^{1}$ Geisinger Precision Health Center, Forty Fort, PA}

Background: Economic evaluation is integral to informed health care decision-making worldwide; however, this research is time-consuming and expensive to conduct. It is especially needed in the rapidly growing and changing field of precision medicine in a form that is relevant to national and local decision-makers. Generic economic evaluation models are proposed as a novel approach to address this critical evidence shortage by transparently adapting published country-specific models to make them generalizable by using available research and allowing users to input local values. The purpose of this study is to apply this approach to develop and test a generic pharmacogenomic economic evaluation model.

Methods: A generic cost-effectiveness model case example was developed to evaluate routine genetic testing in an adult patient population to prevent adverse drug reactions using a published country-specific model. A multidisciplinary international team used a consensus approach to comprehensively review and modify the country-specific model to incorporate generalizable assumptions and parameter values based on evidence reviews and userprovided input values to reflect local conditions. The new generic model was transparently documented, tested and validated using input values and models from multiple countries to compare cost-effectiveness results.

Results: Generic-model base case and probabilistic sensitivity analysis cost-effectiveness results were estimated for implementing a pharmacogenomic test versus two other strategies without the test for multiple countries using country-specific input values. These results were compared to country-specific model results for the same input values. The incremental cost-effectiveness ratios for the generic and country-specific models for three countries and three subpopulations in one country were consistent in terms of whether the pharmacogenomic test strategy was cost-effective at the country-specific threshold value. Differences between the generic and country-specific model results were largely explained by differences in model structure and assumptions.

Conclusion: A generic pharmacogenomic cost-effectiveness model enabling use of local input values is feasible and can offer an efficient and timely value-based decision-making tool. Implementing this approach demonstrates that costeffectiveness analyses can be rapidly performed without extensive training in decision modeling to provide useful evidence for decision-making and facilitate understanding about what conditions can meet cost-effectiveness thresholds.

\section{P8.06}

\section{Health Care Systems Research Network Twin Cohort and its Potential Utility}

Scott Hebbring, ${ }^{1}$ Joseph Boscarino, ${ }^{2}$ Nora Henrikson, ${ }^{3}$ Sharon Fuller, ${ }^{3}$ Mara Epstein ${ }^{4}$

${ }^{1}$ Marshfield Clinic, Marshfield, WI; ${ }^{2}$ Geisinger Health System, Danville, PA; ${ }^{3}$ Group Health Cooperative, Seattle, WA; ${ }^{4}$ Meyers Primary Care Institute, Worcester, MA

Background: Family-based studies have historically been considered a powerful strategy when understanding the etiologies of human disease, especially those influenced by genetics. A gold standard in family-based study design includes twin studies due to the unique genetic relationships between twin siblings, but these unique familial relationships are relatively rare and difficult to recruit. Herein we demonstrate that electronic health record (EHR) systems across multiple Health Care Systems Research Network (HCSRN) sites can identify twin families. We further show the utility of such twin cohorts in research using twins identified in Marshfield Clinic's EHR.

Methods: Twins were predicted by searching for patients who shared a common birthdate and last name along with a common home address, contact information or billing account. The twin prediction algorithm was applied to four different HCSRN sites, including Marshfield Clinic, Group Health Cooperative, Geisinger Health System and Meyers Primary Care Institute. In Marshfield Clinic twins, clinical phenotypes were defined by diagnostic ICD-9 coding. For each phenotype, a measure of familial aggregation and relative risk (RR) was calculated by assessing disease 
concordance in twin families. To further assess potential genetic etiologies, we compared familial aggregation in opposite-sex twins (dizygotic twins) and same-sex twins (enriched for monozygotic twins).

Results: A total of 21,699 families of twins (43,398 individuals) were identified across four HCSRN sites, including 8,242 families of twins from Marshfield Clinic's EHR. Of the 5,598 phenotypes assessed by familial aggregation analysis, 1,222 phenotypes were statistically significant $(\mathrm{P}<8.9 \mathrm{E}-6)$. When simply measuring relative risks across all diseases, $91 \%$ of phenotypes had relative risk $>1$. There was a 4.2-fold enrichment of disease concordance in same-sex twins compared to opposite-sex twins for phenotypes with the largest relative risks. Many of these phenotypes were likely influenced by genetic factors.

Conclusion: This study has generated one of the world's largest cohorts of twins. Unique to this population is the linkage to extensive phenotypic data through an EHR across multiple health care institutions. More broadly, with a significant proportion of diseases aggregating in families of twins, these results may emphasize the significant benefit of incorporating family data when predicting, preventing and treating many diseases for the advancement of precision medicine.

\section{HEALTH INFORMATICS, BIG DATA, VIRTUAL DATA WAREHOUSE}

P9.01

Interactive Visualization of a Patient's Electronic Health Information to Assist Manual Chart Review Using Tableau ${ }^{\circledR}$ Software

David Carrell, ${ }^{1}$ Ladia Albertson-Junkans, ${ }^{1}$ Arvind Ramaprasan, ${ }^{1}$ Andrew Baer, ${ }^{1}$ David Cronkite ${ }^{1}$

${ }^{1}$ Group Health Research Institute, Seattle, WA

Background: Identification of many important health conditions requires synthesis of subtle clinical observations diffusely recorded in electronic health record (EHR) notes, making manual chart review the only viable method of collecting such information. Clinical documentation of problem use of prescription opioids, including abuse and addiction, is an example. A research project we conducted required determining whether problem opioid use was documented in the charts of 2,000 patients receiving chronic opioid therapy during a 9-year observation period. To enhance thoroughness and efficiency, we provided chart abstractors interactive graphical summaries of selected information from each patient's EHR as an abstraction aid. Methods: We used pilot chart reviews and expert opinion to identify four types of information considered useful for identifying clinical documentation of problem opioid use: 1) the timing, type and days' supply of opioid fills; 2) encounters;
3) diagnoses including behavioral health, substance abuse and chronic pain conditions; and 4) mentions in clinical notes of terms related to problem opioid use. We obtained structured data from the virtual data warehouse. We used natural language processing (NLP) to extract information from clinical notes. We created longitudinal graphical displays of each content area using Tableau ${ }^{\circledR}$ (www. Tableau.com), a business intelligence software product that simplifies the creation of graphical representations and realtime exploration of complex data. Graphics were juxtaposed on "dashboards" with shared time scales and drillable details (eg, details of medications fill, text surrounding NLPextracted terms) facilitating a visual synthesis of multiple types of potentially relevant information.

Results: Five experienced chart abstractors used the interactive graphics on one screen and the Epic ${ }^{\circledR}$ EHR interface on a second screen to conduct each chart review. Abstractors reported that the graphics facilitated efficiency through more rapid detection of periods of care in which problem opioid use may be documented (eg, emergency room encounters coinciding with early opioid refills). Particularly valuable was the ability to "see the larger picture" while also being able to drill into the details of specific events.

Conclusion: Graphical visualization of information from EHRs can assist manual abstraction of health conditions when determinations about the presence of those conditions require synthesis of diffusely recorded content in voluminous charts.

P9.03

Developing and Validating a Kaiser Permanente Electronic Health Record Data Transfer to External Research Partner Using Software Designed for Health Data Interoperability

Suzanne Gillespie, ${ }^{1}$ Mary Ann McBurnie, ${ }^{1}$ Vincent Malanga, ${ }^{2}$ Kelly Kirk, ${ }^{1}$ Michael Thornton, ${ }^{1}$ Phillip Crawford, ${ }^{1}$ Weiming $\mathrm{Hu},{ }^{1}$ Renee Petrie, ${ }^{3}$ Mike Stockemer, ${ }^{4}$ Kourtney Davis, ${ }^{5}$ Richard Mularski ${ }^{1}$

${ }^{1}$ Kaiser Permanente Center for Health Research, Portland, OR; ${ }^{2}$ COPD Foundation, Miami, FL; ${ }^{3}$ DatStat Inc., Seattle, WA; ${ }^{4}$ Corepoint Health, Frisco, TX; ${ }^{5}$ GlaxoSmithKline, Collegeville, $P A$

Background: Chronic obstructive pulmonary disease (COPD) affects over 12 million Americans, is responsible for 800,000 hospitalizations each year and is the third leading cause of death in the United States. The continuing lag between clinical practice and treatment makes COPD ripe for patient-centered outcomes research. The COPD Foundation's PCORnet-funded COPD Patient Powered Research Network (COPDPPRN) created a COPD research repository of $>5,000$ participants; 346 of these are represented in Kaiser Permanente's PCORnet PORTAL database and in the COPD Outcomes-based Network for 
Clinical and Effectiveness Translation (CONCERT). Our objective is to develop and validate a scalable methodology for linking and sharing consented patients' comprehensive electronic health record (EHR) data across research data partners.

Methods: A subdatabase containing the EHR data for the subset of COPDPPRN-consented PORTAL patients can be accessed via a RESTful application program interface (API) developed by Kaiser Permanente. Utilizing Corepoint Integration Engine to manage authenticated communication, linkage is verified and data securely transferred to a DatStat shell at COPDPPRN. To validate, the dataset will be generated at DatStat to compare to the CONCERT EHR data as gold standard. Validation includes data accuracy/integrity and identifies potential organizational/systematic/technical errors made during transfer.

Results: Using PCORnet's Common Data Model structure, we populated a subdatabase with COPDPPRN-consented CONCERT patients' EHR data. Kaiser Permanente and DatStat built APIs to provide secure access to the data using the Corepoint technology. The API, called via a secure HTTPS web request, utilizes multifactor authentication. Upon validation of the client credentials and existence of the requested data, the API returns the participant's data in encrypted XML format. Corepoint Health maintains the private key to decrypt the XML data and transfer to DatStat. Data transfers are scheduled, and validation will be completed by early 2017 . Report on the preliminary results is planned for presentation at the Health Care Systems Research Network 2017 Conference.

Conclusion: This work is the first step toward creating a fully functional, scalable system for linking patient data across sources in the PCORnet enterprise to enhance PPRN data for research. Once the API is tested and validated, it can be adapted to access EHR data across PCORnet partners.

\section{P9.04}

\section{Population Insight Tool: A Novel Visual Interactive Query Interface to Virtual Data Warehouse}

Ketan Mane, ${ }^{1}$ Dimitri Buzkov, ${ }^{2}$ Jackie Blank, ${ }^{1}$ Jamila Gul, ${ }^{2}$ Tracy Lieu, ${ }^{2}$ Michael Horberg ${ }^{1}$

${ }^{1}$ Mid-Atlantic Permanente Research Institute, Rockville, MD; ${ }^{2}$ Kaiser Permanente Division of Research, Oakland, CA

Background: In 2013, Kaiser Permanente Northern California (KPNC) developed a virtual data warehouse (VDW) query tool to enable clinicians and investigators to conduct rapid, online simple analyses of key VDW variables without requiring knowledge of SQL or other programming languages. However, the tool's usability has been limited by the user interface: results were returned in a tabular format, which required the user to take time to study the results (or preprocess them) before making interpretations or gathering insights. The Kaiser Permanente Mid-Atlantic States
(KPMAS) and KPNC teams collaborated to overcome this barrier to usability with the design of Population Insight Tool. Our goals were to: 1) Explore new ways to use technology to accelerate users' abilities to query the VDW; and 2) Develop a user-friendly approach to enhance users' abilities to sift through the results for insights.

Methods: Our main challenge was how to handle changes to the output views based on impromptu changes to the user queries. No "off-the-shelf" business intelligence tools (like Tableau ${ }^{\circledR}$ ) could be used - because they all required prior knowledge of user queries to prebuild output views - so we had to design our own solution. Front-end web development framework was used to build a user-friendly query page with drag-and-drop and search-by-keywords features to build queries. The insight tool system architecture was set up to leverage KPNC query engine to query the KPMAS VDW Teradata database and return results. New programs were designed to process the result data and automatically generate a customized dashboard with interactive visuals.

Results: For the clinician/investigator query "Show patient cohort with diagnosis of multiple sclerosis," the insight tool shows results stratified by demographics along with other user-selected query items. Users can directly interact with data to rapidly conduct more detailed analyses and gather insight about specific subgroups such as female, black, nonHispanic, 21-35 age-group, etc.

Conclusion: The insight tool was designed to empower clinicians/investigators to query VDW data directly. It is built to give more flexibility for data analysis with faster access to results in an easy to understand format. It also offers features to drill down to more effectively help the user understand nuances in the data.

P9.05

Early Detection of Heart Failure Using Electronic Health Records: Practical Implications for Time Before Diagnosis, Data Diversity, Data Quantity, and Data Density

Kenney Ng, ${ }^{1}$ Steven Steinhubl, ${ }^{2}$ Christopher deFilippi, ${ }^{3}$ Sanjoy Dey, ${ }^{1}$ Walter Stewart ${ }^{4}$

${ }^{1} I B M$ Research, Cambridge, MA; ${ }^{2}$ Scripps Health, San Diego, CA; ${ }^{3}$ Inova Heart and Vascular Institute, Falls Church, VA; ${ }^{4}$ Sutter Health, Walnut Creek, CA

Background: Using electronic health records (EHR) data to predict events and onset of diseases is increasingly common. Relatively little is known, though, about the tradeoffs between data requirements and model utility.

Methods: We examined the performance of machine learning models trained to detect prediagnostic heart failure in primary care patients using longitudinal EHR data. Model performance was assessed in relation to data requirements defined by: the prediction window length (time before clinical diagnosis), the observation window length (duration 
of observation prior to prediction window), the number of different data domains (data diversity), the number of patient records in the training data set (data quantity), and the density of patient encounters (data density). A total of 1,684 incident heart failure cases and 13,525 gender-, age-category- and clinic-matched controls were used for modeling.

Results: Model performance improved as: 1) the prediction window length decreases, especially when less than 2 years; 2) the observation window length increases but then levels off after 2 years; 3 ) the training data set size increases but then levels off after 4,000 patients; 4) more diverse data types are used, but, in order, the combination of diagnosis, medication order and hospitalization data were most important; and 5) data were confined to patients who had 10 or more phone or face-to-face encounters in 2 years.

Conclusion: These empirical findings suggest possible guidelines for the minimum amount and type of data needed to train effective disease-onset predictive models using longitudinal EHR data.

\section{P9.06}

\section{Deafening Silence: When Is Data That's Not There, Missing?}

Roy Pardee ${ }^{1}$

${ }^{1}$ Group Health Cooperative, Seattle, WA

Background: The virtual data warehouse group has done good work describing subpopulations with knowncompromised data capture. This information is now programmatically available to projects in the form of enrollment variables and has been evaluated once thus far, largely favorably. But that work has been largely descriptive - data rates have been calculated and evaluated against the weak criterion that rates in the known-compromised group be graphically distinguishable from the rest. A next step for this work is to develop informed expectations about what those rates should be, so we can evaluate capture against those benchmarks. This poster describes one small step in this direction.

Methods: We developed a crude model predicting the number of ambulatory visits (AVs) a given enrollee should have on the basis of age and sex. We then used this model to generate predicted visit counts for several groups of enrollees and compared them to the observed counts. We gathered annual AV count data on a cohort of approximately 200,000 people enrolled in 2013 at Group Health Cooperative, whose data capture was most sure. Like much count data, these counts are incredibly skewed. Rather than attempt a sophisticated statistical model, we decided to use median counts by sex and age category. This should generate very conservative predictions as it basically ignores visits by the numerous "frequent flyer" patients whose counts are well above the median. We then used that model to make predictions of the total number of visits for each of several groups of enrollees in each of the 48 months between 2012 and 2015, and calculated the actual AV counts for comparison.

Results: In 4 of the 6 groups evaluated, predictions were indeed substantial underestimates of the observed number of AVs. In 2 smaller groups, however, predictions actually overestimated the total number of AVs. Given the modest nature of the predictions, these 2 groups bear further investigation for sufficiency of data capture.

Conclusion: While study results are unfortunately ambiguous, the work does demonstrate the value of empirically developing a benchmark against which we can evaluate data capture. Doing so can help focus our attention in data quality improvement efforts.

\section{P9.08}

Crusade for Cancer Data: How a Non-SEER Site Populated the Virtual Data Warehouse Tumor Table

\author{
Pamala A. Pawloski, ${ }^{1}$ Jody Jackson, ${ }^{1}$ Amy Butani, ${ }^{1}$ \\ Terese DeFor, ${ }^{1}$ Sheryl Kane ${ }^{1}$
}

${ }^{1}$ HealthPartners Institute, Minneapolis, $M N$

Background: The virtual data warehouse (VDW) tumor table is a valuable data resource available to Cancer Research Network (CRN) researchers to examine the feasibility of potential projects or to conduct research. The Surveillance, Epidemiology and End-Results (SEER) registry provides timely and efficient data for VDW tumor tables. HealthPartners Institute (HPI) is a non-SEER site that has developed alternative approaches (claims-based algorithms, medical record review) to obtain tumor data and participate in cancer-related research. These approaches can identify false-positive cases, be time-consuming and be costly.

Methods: Over the past 15 years, HPI has undertaken numerous activities to identify a viable electronic data source to populate the VDW tumor table and thereby more readily participate in multisite studies within the CRN. These activities included two internally-funded capacitybuilding projects. The first, conducted in 2009, examined the ability to connect with the population-based cancer registry Minnesota Cancer Surveillance System, maintained by the Minnesota Department of Health (MDH), to identify cancer cases with specific diagnostic and treatment criteria preparatory to research. In 2011, our second project explored linking with the electronic registry system (ERS) at an HPIowned hospital, Regions. More recently, HPI increased its tumor registry case ascertainment with the inclusion of care centers in Eastern Minnesota and Western Wisconsin as well as the merging of another health system, Park Nicollet.

Results: The 2009 feasibility project demonstrated that MDH was able to link $79 \%$ of cases identified by HPI claims data. Nonmatches occurred from misclassification by HPI-created algorithms, patients not living in 
Minnesota, or patients with cancers different from those identified by HP records. Concordance for determining eligibility (stage, date of diagnosis) was high, but not 100\%. The 2011 feasibility project demonstrated that HPI programmers can access Regions cancer registry data directly via the ERS similar to other data sources (eg, Clarity, Epic) through procurement of a software license and training. The project programmer extracted registry cases from ERS, mapped data elements to variables outlined in the VDW tumor tables and performed quality assurance checks provided by the VDW tumor work group. Thus, ERS data is currently our electronic source for the VDW tumor table. Following the expansion of the HPI care network to include Lakeview Hospital and three Cancer Center of Western Wisconsin sites (Westfields Hospital and Clinic, Amery Hospital and Clinic, Hudson Hospital and Clinic) along with the 2014 merger of HPI and Park Nicollet organizations, HPI is currently extracting tumor data from the ERS at these sites and Park Nicollet Methodist Hospital. Ongoing efforts to enhance the HPI tumor file include obtaining any available tumor information from MDH for all HPI patients and members.

Conclusion: After time and vigorous exploration, HPI's tumor data is part of the CRN Cancer Counter. More recent activities have enriched this data. HPI is in a better position to not only conduct internal cancer research, but also to participate in multisite studies. A visual timeline of the activities undertaken to identify and connect with tumor data sources, as well as the challenges, successes and proposed future work, will be presented.

\section{HEALTH PLAN COVERAGE AND POLICY}

\section{P10.01 \\ Factors Associated With Participations and Goals Achievement in an Employee Wellness Program Offering Biometric Screenings and Premium Discounts}

\section{Zhi Geng, ${ }^{1}$ Daniel Maeng ${ }^{1}$ \\ ${ }^{1}$ Geisinger Health System, Danville, PA}

Background: Since 2012, Geisinger Health System (GHS) has redesigned its employee wellness program MyHealth Rewards by requiring biometric screenings and goal achievements for blood pressure, body mass index, glucose and cholesterol levels to be eligible for premium discount in subsequent year. The objective of this study is to determine what baseline employee characteristics were associated with MyHealth Rewards participations and goals achievements.

Methods: Claims data from 2011 to 2015 were obtained from Geisinger Health Plan, restricting to continuously enrolled members. Four mutually exclusive cohorts were identified: GHS employees who met goals in all years
(Group 1, n=2,842); GHS employees who met goals some years (Group 2, n=3,999); GHS employees who never joined MyHealth Rewards (Group 3, $\mathrm{n}=4,398$ ); and nonGHS employees $(n=24,061)$. A multinominal logit model was used to estimate the probability of being in one of the groups as a function of demographics, chronic conditions and utilization at the baseline (2011).

Results: Compared with non-GHS employees, female employees were less likely to never participate (Group 3; odds ratio $[\mathrm{OR}]$ : $0.61, \mathrm{P}<0.01)$ and more likely to meet goals in all years (Group 1; OR: 2.7, P<0.001). Employees with more chronic conditions or above 45 at the baseline were less likely to be in Group 1 (OR: $<0.08, \mathrm{P}<0.01$ ). Specialist visits at the baseline were associated with higher likelihood of being in Group 1 (OR: 1.28, $\mathrm{P}<0.01$ ), but emergency department visits were associated with the opposite (OR: $0.77, \mathrm{P}<0.01$ ).

Conclusion: Employees' preprogram baseline characteristics predict the employee participation and goal achievement patterns in subsequent years. Specifically, there was a self-selection of employees who were female, younger and healthier prior to the program implementation. This suggests that employees' baseline characteristics should be taken into account in designing and evaluating similarly designed employee wellness programs.

P10.02

Impact of Employee Wellness Program on Health Outcome: A Propensity Score-Matched Analysis

\section{Zhi Geng, ${ }^{1}$ Daniel Maeng ${ }^{1}$ \\ ${ }^{1}$ Geisinger Health System, Danville, PA}

Background: Since 2012, Geisinger Health Plan has redesigned its employee wellness program MyHealth Rewards (MHR) by requiring biometric screenings and goal achievements for blood pressure, body mass index, glucose and cholesterol levels to be eligible for premium discount in subsequent year. This study is designed to evaluate the impact of MHR on stroke and myocardial infarction.

Methods: Claims data from 2011 to 2015 were retrieved from Geisinger Health Plan, restricting to continuously enrolled members. Four mutually exclusive cohorts were identified: Geisinger Health System (GHS) employees who met goals (Group 1); GHS employees who met goals some years (Group 2); GHS employees who never joined MHR (Group 3); and non-GHS employees (Group 4). After oneto-one propensity score matching was used to balance the baseline characteristics (eg, sex, age, plan type, chronic condition, utilizations), 11,239 GHS employees (Group 1: 2,842; Group 2: 3,999; Group 3: 4,398) and matched non-GHS employees of equal numbers were included for analyses. Kaplan-Meier method and Cox proportional hazards models were used to estimate the difference in time to first stroke or myocardial infarction between GHS and 
non-GHS employees.

Results: After one-to-one propensity score matching, both GHS and non-GHS cohorts had similar baseline characteristics; yet, GHS employees in Groups 1 and 2 had a consistently higher probability of an event-free outcome at each time period since 2011 than the nonGHS employees, both in terms of stroke and myocardial infarction. However, the differences were not statistically significant. The estimated hazard ratios associated with the GHS employee status across all subgroups were 0.75 $(\mathrm{P}=0.14)$ for stroke and $0.91(\mathrm{P}=0.39)$ for myocardial infarction. In contrast, Group 3 (never enrolled) has higher risk of myocardial infarction (hazard ratio: $1.18, \mathrm{P}=0.30$ ) but lower risk of stroke (hazard ratio: $0.68, \mathrm{P}=0.25$ ).

Conclusion: Although the estimated MHR effects were not statistically significant, the results are consistent with the expectation that MHR may help prevent adverse health outcomes. This expectation is further supported by a separate analysis showing long-term cost of care savings associated with MHR.

\section{P10.03}

Impact of a Biometric Screening and Premium Incentive-Based Employee Wellness Program on Cost of Care and Utilization

\section{Daniel Maeng, ${ }^{1}$ Zhi Geng ${ }^{1}$ \\ ${ }^{1}$ Geisinger Health System, Danville, PA}

Background: Since 2012, Geisinger Health System (GHS), in collaboration with Geisinger Health Plan, has redesigned its employee wellness program MyHealth Rewards by requiring biometric screenings and achievement of a set of predefined goals for blood pressure, body mass index, glucose and cholesterol levels to be eligible for premium discounts in the subsequent year. GHS employees can voluntarily choose to enroll in MyHealth Rewards in a given year, and to qualify for the premium discounts they are required to meet all the biometric goals prior to November of the same year.

Methods: This study evaluates the impact of MyHealth Rewards on the employee costs of care and utilizations. A difference-in-differences approach using a generalized linear model was used to estimate the observed and expected values in terms of per-member-per-year (PMPY) total medical allowed amounts and health care utilization. The intervention group consisted of GHS employees $(n=11,239)$ and the comparison group consisted of nonGHS employees $(n=24,061)$ who remained Geisinger Health Plan commercial members throughout the study period (2011-2015) and did not switch between the two groups. 2011 was defined as the preintervention period.

Results: For medical costs excluding prescription drugs, there were no cost savings PMPY in 2012 and only a minor cost savings of $\$ 230(5.0 \%, \mathrm{P}=0.183) \mathrm{PMPY}$ in 2013. In
2014 and 2015, however, there were significant cost savings of $\$ 412(8.4 \%, \mathrm{P}=0.026)$ and $\$ 480(8.8 \%, \mathrm{P}=0.024) \mathrm{PMPY}$, respectively. These savings were driven by reductions in outpatient costs, which increased from $\$ 112$ (5.8\%) to $\$ 232$ (9.0\%) in 2014 and $2015(\mathrm{P}=0.1)$.

Conclusion: A similarly designed employee wellness program that provides premium discounts for meeting a predefined set of biometric goals may reduce long-term cost of care among the employee population.

\section{P10.04 \\ "Apple or PC:" Creating a Patient-Centered Tool to Help Medicare Beneficiaries Choose Prescription Drug Plans}

Ming Tai-Seale, ${ }^{1}$ Cheryl Stults, ${ }^{1}$ Amy Meehan, ${ }^{1}$ Mary Kate Bundorf ${ }^{2}$

${ }^{1}$ Palo Alto Medical Foundation Research Institute, Mountain View, CA; 'Stanford University School of Medicine, Stanford, $C A$

Background: Choosing a Medicare Part D prescription drug plan is complicated; there are many plans from which to choose, and the appropriateness of the coverage varies depending on medication needs and patient preferences. It's been well documented that Medicare beneficiaries report frustration with choosing a prescription plan due to the large number of options and, in focus group research, we found that many older adults expressed a desire for expert guidance. We designed a patient-centered online Part D plan selection tool (CHOICE) to simplify their choice process and provide personalized, expert recommendations. In this study, we examined patient use of the new tool and the existing decision tool available at Medicare.gov.

Methods: This ethnographic comparative usability study engaged 44 patients during the 2016 open enrollment period (October 15-December 7, 2015). Participants were observed as they chose their drug plan using Medicare.gov and one of three versions of CHOICE. The versions varied in the amount of expert guidance provided on drug plans. The participants completed an exit survey regarding their experience. Descriptive statistics were used to analyze the survey data. The observations were video-recorded and field notes were analyzed thematically.

Results: One usability tester likened CHOICE as an Apple product and Medicare.gov a PC product. User survey results suggest that the time-on-task was much shorter when using CHOICE. Participants were significantly more satisfied with the process of choosing a Part D drug plan using CHOICE (44\%) than with using Medicare. gov $(14 \%) ; 46 \%$ of participants strongly agreed that they understood the information in CHOICE and $45 \%$ strongly agreed that CHOICE was easy to use compared to $20 \%$ and $16 \%$, respectively, when using Medicare.gov $(\mathrm{P}<0.05)$. Participants randomized to two arms of CHOICE with 
increasing levels of expert guidance were more likely to choose a drug plan other than their current plan than those using Medicare.gov $(\mathrm{P}<0.15)$.

Conclusion: Many user-centered features of CHOICE improved patient experience and enabled them to choose plans more consistent with expert recommendations. We are mounting a large-scale three-arm randomized controlled trial in the 2017 open enrollment period to test CHOICE.

\section{HEALTH SERVICES DELIVERY AND UTILIZATION}

\section{P11.01 \\ Adherence and Persistence With Therapy Among Newly Prescribed Fibromyalgia Patients in Israel}

Dahlia Weitzman, ${ }^{1}$ Dana Ben-Ami Shor, ${ }^{2}$ Varda Shalev, ${ }^{1}$ Howard Amital, ${ }^{3}$ Gabriel Chodick ${ }^{1}$

${ }^{1}$ Maccabitech, Maccabi Healthcare Services and Sackler Faculty of Medicine, Tel Aviv University, Tel Aviv, Israel; ${ }^{2}$ Department of Gastroenterology, Sheba Medical Center, Tel-Aviv University, Tel Hashomer, Israel; ${ }^{3}$ Department of Medicine "B", Zabludowicz Center for Autoimmune Diseases, Sheba Medical Center, Tel Hashomer, Israel

Background: Fibromyalgia is a chronic debilitating disorder affecting over $4 \%$ and $\sim 1.5 \%$ of the female and male adult population worldwide, respectively. The study aimed to assess 1-year persistence and adherence with therapy among newly prescribed fibromyalgia patients and to identify factors associated with therapy discontinuation. Methods: Included were adult members of Maccabi Healthcare Services, a 2 million-member health maintenance organization in Israel, diagnosed with fibromyalgia between 2008 and 2011. Persistence and adherence with anticonvulsants and antidepressants were analyzed among newly prescribed fibromyalgia patients by comparing those with $\geq 2$ medication dispenses versus 1 or none and by examining time to treatment discontinuation $(\geq 120$ days) and proportion of days covered (PDC) with medication during the year following first dispense. Logistic regression models were constructed for multivariable analyses.

Results: The majority of 3,932 eligible fibromyalgia patients were female $(90 \%)$, and $41.2 \%$ were already prescribed fibromyalgia medications prior to diagnosis. Of the remaining 2,312 patients, 56.1\% were issued a prescription, $45.0 \%$ had at least one medication dispensed in the year following diagnosis and only $28.8 \%$ had at least one additional dispense within a year from the first dispense. One-year treatment discontinuation was highest for tricyclic antidepressants (91.0\%) and lowest for SSRI/ SNRI antidepressants (73.7\%). In multivariate analysis, having $\geq 2$ dispensed fibromyalgia medications versus 1 or none was associated with baseline anxiety and depression (odds ratio: 1.86, 95\% confidence interval $[\mathrm{CI}]$ : 1.33-2.60, $\mathrm{P}<0.001$ ) and with migraines (odds ratio: $1.34,95 \% \mathrm{CI}$ : $1.03-1.73)$. Half of the patients (50.9\%) had $<20 \%$ of days covered by any medication during the year, and only $11.6 \%$ were adherent ( $\mathrm{PDC} \geq 80 \%$ ). Socioeconomic status (SES) was the only factor associated with having $\mathrm{PDC} \geq 80 \%$ : $7.1 \%$ vs $14.4 \%$ were adherent in the lowest and highest SES quintiles, respectively (P for trend: 0.017).

Conclusion: Among newly prescribed fibromyalgia patients, use of fibromyalgia medications is remarkably low, possibly related to high cost, low effectiveness and/or intolerability. Additional research is required to investigate whether these patients utilize nonpharmacological therapies and to what extent they are effective. Nonetheless, it is important to endorse adherence intervention initiatives in order to lower the burden and costs for patients and providers alike and to improve the quality of life of the patients.

\section{P11.02}

Do Preventive Visits Among Seniors Lead to Better Completion of Recommended Preventive Services?

\section{Sukyung Chung, ${ }^{1}$ Wang Xiang, ${ }^{1}$ Robert Romanelli ${ }^{1}$}

${ }^{1}$ Palo Alto Medical Foundation Research Institute, Palo Alto, $C A$

Background: Under the Affordable Care Act, the annual preventive visit became fully covered by Medicare. We assessed whether the use of preventive visit, as compared to frequent use of routine nonpreventive primary care visit, is associated with higher completion rates of recommended preventive care services.

Methods: Primary care patients (aged $65-85$ years) in a large, mixed-payer ambulatory care organization between 2011 and 2014 were identified $(\mathrm{N}=184,576)$. We examined preventive care services recommended by U.S. Preventive Services Task Force guidelines or the National Committee for Quality Assurance. Services were categorized as: 1) Preventive Screening - colorectal cancer screening, breast cancer screening; 2) Management of Chronic Conditions - annual monitoring for patients on persistent medications (ACE inhibitors/ARBs or diuretics), coordinated diabetes care; and 3) Preventive Counseling - smoking cessation counseling, discussion of end-of-life care planning. We estimated the likelihood of completing each service by preventive visit status (yes/no) and the frequency of nonpreventive primary care visits. We used a mixed-effect multivariate logistic regression, controlling for patient demographic and clinical characteristics and provider characteristics, with patient random effects to take into account clustering across multiple observations per patient. Odds ratios (OR) were generated. A statistical significance level of 0.01 was used. 
Results: Seniors who had a preventive visit, versus those who did not, were more likely to have completed recommended services for Preventive Screening (OR: 1.77-1.85), Management of Chronic Conditions (OR: 1.32-1.48) and Preventive Counseling (OR: 3.04-3.95). The likelihood of completing preventive services increased with the frequency of nonpreventive primary care visits for some services, but to a much smaller degree, for one (OR: 1.21-1.25), two (OR: 1.10-1.95), three (OR: 1.23-2.29) and four or more (OR: 1.29-2.92) versus no nonpreventive visit. Predicted probability of completing "discussion of end-oflife planning," for example, was larger with one preventive visit (without nonpreventive visit) $(31.12 \%)$ than with $4+$ nonpreventive primary care visits (without preventive visit) $(21.95 \%)$.

Conclusion: Seniors with preventive visits were more likely to complete recommended preventive services than those who make frequent nonpreventive visits. This was particularly prominent for Preventive Counseling services, which are time-consuming and thus difficult to be handled during routine nonpreventive visits. Annual preventive visit may be necessary for improved preventive care for seniors.

\section{P11.03}

\section{Emergency Department Use in a U.S. Health Care System Before and After Implementation of the Coverage Provisions of the Affordable Care Act}

\author{
Lisa Dean-Gilley, ${ }^{1}$ Anjali Franco, ${ }^{1}$ Lydia Xu, ${ }^{1}$ Jessica \\ Liu, ${ }^{1}$ Sarah Robinson, ${ }^{1}$ Alice Pressman ${ }^{1}$
}

\section{${ }^{1}$ Sutter Health, Walnut Creek, $C A$}

Background: Overcrowding of hospital emergency departments (EDs) presents a major public health issue across the United States. Overcrowding has been linked to decline in quality of care and patient satisfaction, decreased revenue and increased patient mortality. In January 2014, the full implementation of the Affordable Care Act (ACA) extended health coverage to 32 million Americans. The literature has subsequently reported a post-ACA rise in ED utilization, but early analysis of two hospitals in the Sutter Health system demonstrated a delayed effect. We sought to characterize the longitudinal changes in ED utilization at 21 EDs across Sutter Health before and after ACA.

Methods: We reviewed demographic and hospital accounts data from the California Office of Statewide Health Planning and Development for all 21 Sutter Health EDs throughout Northern California. We compared utilization and examined trends over time, from 2010 to 2015. We categorized five types of trends over time as follows: $\mathrm{NC}=$ no change, $\mathrm{CI}=$ constant increasing slope, $\mathrm{CD}=$ constant decreasing slope, DI = delayed effect until 2014 with increase thereafter, and DD = delayed effect until 2014 with decrease thereafter.
Results: Across the system from January 2010 to December 2015, 15 (71\%) EDs followed a DI pattern, 4 (19\%) a CI pattern, 1 an NC pattern, and 1 was unclassified. For Medicaid patients, 15 (71\%) EDs followed a DI pattern. For all hospitals, self-pay patients followed DD patterns of utilization. Use by Medicare patients primarily followed an $\mathrm{NC}$ pattern $(\mathrm{n}=17,81 \%)$. Overall utilization increased by $20 \%$ during the study period. This was driven by a $6 \%$ increase in the first 4 years, followed by $12 \%$ increase for the last 2 years of the study period.

Conclusion: ED use across the Sutter Health system increased after the implementation of the ACA. The escalation in annual ED visits was driven by a sharp increase after the expansion (full implementation). In fact, utilization increased 4 times faster in the 2 years post-full implementation than in the first 4 years of the initial implementation, and was attributable to a shift in uninsured patients (self-pay) gaining Medicaid or commercial insurance coverage. Further research is needed to determine how these changes affect costs and the possible implications for Sutter Health EDs.

\section{P11.04}

\section{Correlates of High Performance After Implementing Lean Redesigns in Primary Care}

Dorothy Hung, ${ }^{1}$ Su-Ying Liang, ${ }^{1}$ Caroline Gray, ${ }^{1}$ Meghan Martinez, ${ }^{1}$ Michael Harrison, ${ }^{2}$ Harold Luft ${ }^{1}$

${ }^{1}$ Palo Alto Medical Foundation Research Institute, Mountain View, CA; ${ }^{2}$ Agency for Healthcare Research and Quality, Rockville, $M D$

Background: This mixed-methods study leverages activities of a large ambulatory delivery system that used Lean techniques to redesign primary care workflows. Though Lean has been shown to improve quality and reduce costs, there are no studies examining organizational or provider characteristics associated with Lean improvements. We describe contextual features of primary care departments that demonstrated greatest performance improvements after implementing Lean redesigns.

Methods: We identified departments with highest postLean improvements on performance metrics, including: efficiency, productivity, patient satisfaction, and clinical quality. Changes in each metric were based on segmented regressions that modeled: 1) immediate change following Lean implementation, and 2) gradual change over time. Departments were identified as high performing if either of these charges were positive and statistically significant, with no decreases in other metrics. We examined bivariate associations between high-performing departments and contextual variables gathered from a survey of 860 physicians and staff, with responses aggregated to the department level. Findings were triangulated with qualitative data gathered from nearly 200 interviews and focus groups with physicians, staff and organizational leaders. 
Results: High performance was associated with a high perceived history of change, as compared with low change (29.5\% vs $18.9 \%, \mathrm{P}<0.05)$. High-performing departments also were more likely to have lower levels of provider burnout compared to those with higher burnout (30.8 vs $19 \%, \mathrm{P}<0.05)$. These findings are consistent with qualitative findings describing Lean changes as supporting improved care delivery: "We want to be getting our work done in a good clip and taking care of our patients ... Lean gave us the framework to improve that." While burnout was prevalent, hope was expressed that Lean redesigns may assist with workload: "Just grinding out patients ... and trying to keep up with documenting ... volume of e-messages, phone calls, labs ... it's just daunting. It feels like emptying the ocean with a teaspoon; it's nonstop, and to think 'oh, I might have help...?."

Conclusion: Perceptions of change and provider burnout were associated with changes in performance metrics. Findings suggest that Lean redesigns may have a dual effect on performance by not only altering work processes, but potentially ameliorating burnout and allowing providers to provide more effective patient care.

\section{P11.05 \\ Sustainment of Lean Redesigns in Primary Care Clinics}

Dorothy Hung, ${ }^{1}$ Caroline Gray, ${ }^{1}$ Su-Ying Liang, ${ }^{1}$ Meghan Martinez, ${ }^{1}$ Michael Harrison ${ }^{2}$

${ }^{1}$ Palo Alto Medical Foundation Research Institute, Mountain View, CA; '2Agency for Healthcare Research and Quality, Rockville, $M D$

Background: While Lean is rapidly increasing in popularity within the health care sector, most studies to date focus on discrete and narrow uses of Lean techniques to solve isolated problems. In this study, we examine the implementation and sustainment of transformative Lean workflow redesigns nearly 5 years after being introduced in all primary care clinics of a large ambulatory care delivery system.

Methods: Lean redesigns were implemented in phases across 17 clinic locations of the delivery system - first in one pilot site, then in three "beta" sites, and finally all remaining "gamma" clinic sites. Our mixed-methods analysis was guided by the Consolidated Framework for Implementation Research modified for process redesign. Findings are based on 57 in-depth interviews with physicians, staff and clinic leaders, and survey responses from 860 primary care physicians and staff (74.8\% response rate).

Results: We found that clinics' initial approach to implementing Lean redesigns was critical to later sustainment. The pilot site experienced greatest successes, with diminishing results observed in gamma clinics. Facilitators of sustainment included the presence of skilled and charismatic Lean change agents, open/democratic leader engagement with frontline staff and physicians, and simple work designs that were easily adopted and maintained. Despite initial resistance, the most sustained and widely appreciated Lean changes included colocation of medical assistant/physician care teams, and " $5 \mathrm{~S}$ " reorganization and standardization of examination room supplies. According to surveys on specific workflow redesigns, care teams reported using daily huddles most of the time to carry out their work and agenda setting by medical assistants for the majority of patient visits.

Conclusion: This study highlights areas in which leaders can focus their efforts to enhance successful Lean change initiatives. Program implementers may find it easier to introduce Lean in phases rather than at a single time point. However, it is critical for sustainment that at every phase, frontline physician and staff are equally engaged in the design and implementation of new work designs.

\section{P11.06 \\ Provider Perspectives on Behavioral Health Navigation Program for Adolescents}

\section{Martina $\mathrm{Li}^{1}$}

${ }^{1}$ Palo Alto Medical Foundation Research Institute, Mountain View, $C A$

Background: This research evaluation is for an adolescent behavioral health $(\mathrm{BH})$ quality improvement project within a multispecialty health care delivery system. Approximately $20 \%$ of U.S. adolescents have a mental health condition, thus underscoring the $\mathrm{BH}$ needs of this population. Best practices call for early detection of conditions by primary care providers (PCPs) and coordinated care aimed at managing conditions. Results from baseline PCP surveys and interviews showed that while confident about identifying adolescent $\mathrm{BH}$ needs, PCPs face challenges in providing necessary and appropriate treatment for their patients. In an effort to support PCPs and provide improved quality of $\mathrm{BH}$ care in primary care, a navigation program was developed to facilitate referrals from PCPs to $\mathrm{BH}$ specialists for adolescents. We asked PCPs about their experience with the program.

Methods: Pediatric and family medicine PCPs with access to the adolescent $\mathrm{BH}$ navigation program were surveyed and interviewed.

Results: Over 3,400 referrals have been made to the navigation program to date. Of 150 PCPs surveyed, 106 (70.7\%) stated that they have used the navigation program. Their reasons for use include: for help with finding $\mathrm{BH}$ providers who accept patients' insurance (96.2\%), to ensure patients receive timely $\mathrm{BH}$ care $(83.0 \%)$, and for help with determining appropriate type of $\mathrm{BH}$ provider or care for patients $(42.6 \%) .87 .7 \%$ of PCPs believed that navigation has enhanced their clinical care. Additionally, PCPs rated the program positively on: the referral process to navigation 
(90.6\%), communication with navigators (87.7\%), and overall experience with navigation (89.6\%). However, PCPs commented through surveys and interviews that the navigation program could be improved. Some PCPs prefer having more feedback from navigators regarding patient referral status. Most noticeably, PCPs are concerned about delayed response time. They recognize that increasing demand for the program may be causing a slowdown; still, PCPs would like for navigators to process their referrals in a more timely manner.

Conclusion: Support needed by PCPs is evidenced by the demand of this $\mathrm{BH}$ navigation program. PCPs indicated that the program is a valuable service and offered suggestions for improvement. We will use their feedback to further refine our program to support PCPs and their adolescent patients.

\section{P11.07}

\section{Evaluating Unwarranted Variation in Treatment Patterns Using Unblinded Data}

\section{Satish Mudiganti, ${ }^{1}$ Michael van Duren, ${ }^{1}$ Sylvia Sudat, ${ }^{1}$ Walter Stewart ${ }^{1}$}

\section{${ }^{1}$ Sutter Health, Walnut Creek, $C A$}

Background: Unwarranted practice variation is an issue for most health care systems and is sometimes caused by external factors. We examined the peer group Variation Reduction (VR), a program in which clinicians are shown data about their treatment patterns alongside other clinicians and are usually surprised by the variation. The information drives a desire to understand the variation in how they care for their patients and can lead to a change in clinician behavior. We sought to evaluate VR projects aimed at reducing brand name prescribing and increasing appropriate documentation of end-of-life (EOL) wishes in a large integrated health care system.

Methods: In this physician-controlled process, providers reviewed and discussed data in a safe environment ruled by principles that confirm confidentiality, safety and group control. Verbal consent of all group members is required for each VR step, including topic selection and discussion of relevant data. For analyzing projects, we chose a pre-post parallel design. Providers having at least 12 months of data prior to the VR program and 3 months of data after the VR program were included in the analysis. The control group was comprised of clinicians not involved in the VR program who had enough data for the selected projects. Orders in the 3 months after the VR program were compared to the 12 months before the VR program for the intervention and control physicians. Repeated measures within clinicians were modeled using generalized estimating equations. Standard errors were estimated using nonparametric bootstrap with 1,000 iterations, and $95 \%$ confidence intervals $(\mathrm{CI})$ are reported.
Results: Postintervention, accounting for the 12-month preperiod, physicians in the intervention group prescribed generics for corticosteroids and nasal steroids significantly more than physicians in the control group, and EOL wishes were documented more frequently in the intervention group: 9.4\% (CI: 5.4-14.3) corticosteroids; 3.2\% (CI: 1.84.6) nasal steroids; $0.5 \%$ (CI: $0.2-0.8$ ) documenting EOL wishes.

Conclusion: For all three projects presented, the VR intervention was associated with a reduction in variation in the practices. These results indicate that clinician behavior can be changed with a peer-group process, without the need for additional incentives.

P11.08

Risk of Major Gastrointestinal Bleeding Among Dabigatran Users: A Population-Based, New-User, Self-Controlled Study

Wenze Tang, ${ }^{1}$ Hsien-Yen Chang, ${ }^{2}$ Meijia Zhou, ${ }^{3}$ Sonal
Singh ${ }^{4}$

${ }^{1}$ George Washington University, Washington, DC; ${ }^{2} J o h n s$ Hopkins University, Baltimore, MD; ${ }^{3}$ University of Pennsylvania, Philadelphia, PA; ${ }^{4}$ Meyers Primary Care Institute, Worcester, $M A$

Background: Dabigatran is an oral anticoagulant approved for stroke prevention among patients with atrial fibrillation. Our objective was to evaluate the real-world risk of major gastrointestinal bleeding among dabigatran users.

Methods: We adopted a new-user, self-controlled case series design to reduce confounding by indication. We sampled 1,215 eligible participants who were continuous adult commercial users in a large administrative claims database between July 1, 2010, and March 31, 2012, with use of dabigatran and at least one major gastrointestinal bleeding episode. We used a conditional Poisson regression to estimate incidence rate ratio (IRR) after adjusting for various confounders.

Results: The population consisted of $64.49 \%$ of male and $61.81 \%$ elderly patients (ie, age $\geq 65$ years). After adjustment for time-variant confounders, including bleeding-related comorbidities and concomitant medication use, the incidence rate of major gastrointestinal bleeding was $13 \%$ higher during dabigatran risk period compared with the nonexposed period $(95 \%$ confidence interval [CI]: 0.99-1.27). There was no significant difference between periods of dabigatran and warfarin use (IRR: 1.02, 95\% CI: 0.77-1.34).

Conclusion: This study should help the clinicians prescribe the appropriate anticoagulant for patients together with other studies on the efficacy, effectiveness and safety of anticoagulant. 
P11.09

Development of an Algorithm to Prospectively Identify Palliative Care-Eligible Patients From the Electronic Health Record

Sylvia Sudat, ${ }^{1}$ Kathy Blanton, ${ }^{1}$ Jessica Hanserd, ${ }^{2}$ Kenneth Rosenfeld, ${ }^{3}$ Anjali Franco, ${ }^{1}$ Shruti Vaidya, ${ }^{1}$ Alice Pressman ${ }^{1}$

${ }^{1}$ Sutter Health, Walnut Creek, CA; ${ }^{2}$ Sutter Care at Home, Emeryville, CA; ${ }^{3}$ California Pacific Medical Center, San Francisco, $C A$

Background: For referral-based health care programs, enrollment is usually triggered by a negative health event. This can mean that referral occurs late in the course of illness, even if patients would have benefited from earlier enrollment. In addition, a referral-based model gives little information about the total number of patients in need and provides no opportunity for outreach to better serve potentially eligible patients. The ability to prospectively identify appropriate patients using data from the electronic health record (EHR) presents a possible answer to these challenges. We present an evaluation of an EHR-based prospective identification method focused on palliative careeligible patients.

Methods: Sutter Health is a health system in Northern California with 24 hospitals, more than 5,000 physicians and an embedded home health and hospice organization. Using Medicare fee-for-service (FFS) claims data from our decedents from 2009 to 2014, we created a preliminary algorithm to identify patients who were eligible for a palliative care program. We then validated this identification system against a cohort of enrolled patients. Work is currently underway to generalize this algorithm using data from Sutter Health's EHR (Epic) to prospectively identify palliative care-appropriate patients. We plan to update our current results and perform a more rigorous analysis with access to both referral and enrollment data. We also are incorporating clinician chart reviews to identify false positives.

Results: Use of this algorithmic approach on a retrospective cohort of deceased Medicare FFS palliative care enrollees resulted in identification of $93 \%$ of enrolled patients, though it was not possible to determine the number of patients identified who were not appropriate. The expanded EHRbased analysis will supplement this gap and also provide a more comprehensive view of algorithm performance.

Conclusion: Prospective identification of palliative careeligible patients from the wealth of EHR data presents an important opportunity to use data to better serve our patients. An effective identification system also could be an important tool for clinicians and managers in identifying underserved populations, allocating program resources and planning for program growth.
P11.10

Fidelity Evaluation of a Care Coordination Solution for Integrative Medicine: Usage and User Acceptance

Shruti Vaidya, ${ }^{1}$ Ridhima Nerlekar, ${ }^{1}$ Chelsea Lunders, ${ }^{1}$ Margot Markman, ${ }^{1}$ Alice Pressman ${ }^{1}$

${ }^{1}$ Sutter Health, Walnut Creek, CA

Background: Care coordination is an important aspect of patient care, especially for integrative health, which includes many types of providers on a care team. We developed and piloted an application (Trellis) designed to improve care coordination for patients at the Institute for Health and Healing, an integrative medicine specialty care clinic in San Francisco, California. Trellis is built to improve transparency, awareness and communication between care-team members. We evaluated the usage and user acceptance of Trellis to determine whether we should continue the dissemination process by creating the next version.

Methods: We implemented Trellis and trained the 32 members of the care-team staff. We measured usage by counting active sessions and number of times each feature was accessed. We stratified by provider modality and role, and because most of the practitioners are part-time employees, we weighted usage estimates by hours worked per week. We held a focus group to measure user acceptance. When low usage was identified, we made changes to the application and workflow to improve utilization.

Results: Through July 2016, Trellis has impacted care for 383 (27\%) patients with encounters at the Institute for Health and Healing. Overall, $43 \%$ of the usage can be attributed to medical practitioner, $20 \%$ to allied health practitioners and $37 \%$ to administrative staff. Trellis usage evaluation identified 4 super-users and 7 under-users. Changes to the system, including training, bug fixes and onsite support, resulted in better short-term usage, but not long-term. Trellis helped to track referrals; $72 \%$ of practitioners refer to themselves and about $28 \%$ to specialists of other modalities. Focus groups identified barriers such as culture, workstation set-up and uncertainty about overall purpose.

Conclusion: Trellis adoption was low, only used for onefourth of patient encounters, but there was enthusiasm for future versions and future use. Weekly usage combined with focus group data indicated that the enthusiasms to use the application could not be maintained over time due to cultural barriers. With this early evaluation, we have provided a foundation of information on which to design future versions of the application. 


\section{HEALTH CARE DISPARITIES}

P12.01

\section{Prevalence of Social Determinants of Health in a Large Non-Safety Net Health Plan Membership}

\section{Nancy P. Gordon ${ }^{1}$ \\ ${ }^{1}$ Kaiser Permanente Division of Research, Oakland, CA}

Background: Health plans are exploring how to assess and address social determinants of health (SDOHs) in their socioeconomically diverse memberships. We used survey data to examine prevalence of SDOHs among adults in Kaiser Permanente Northern California.

Methods: SDOH prevalence among members aged 25-79 years was estimated using pooled weighted data from 2011 and 2014 Member Health Surveys (N>22,000). SDOHs included: educational attainment, income, financial worry, reduced medical care/medication use/fruit and vegetable consumption due to cost, chronic stress, caregiver responsibilities, life satisfaction, harassment/discrimination, concern about neighborhood violence, preventive dental care, health beliefs, and ability to use the internet. Estimates were made for ages 25-64 (NS) and 65-79 (S) and evaluated for sex and racial/ethnic differences.

Results: Overall, $19 \%$ (18\% of NS, $27 \%$ of S) had low educational attainment ( $\leq$ high school graduate), and $49 \%$ ( $51 \%$ of NS, $41 \%$ of S) were college graduates. About $9 \%$ ( $7 \%$ of $\mathrm{NS}, 21 \%$ of $\mathrm{S}$ ) had a household income (HHI) of $\leq \$ 25,000$ and $15 \%$ (13\% of NS, $35 \%$ of senior women, $20 \%$ of senior men) an HHI of $\leq \$ 35,000$. In the past year: $13 \%$ ( $15 \%$ of NS, $6 \%$ of S) had forgone or delayed medical care, $7 \%$ (no age difference) had used less medication, and 9\% had consumed less fruit/vegetables than they would have, each due to cost; $28 \%$ ( $31 \%$ of NS, $15 \%$ of S) had worried a great deal about their financial situation; $6 \%$ (7\% of NS, $3 \%$ of S) had worried about neighborhood violence; $17 \%$ ( $23 \%$ of NS women, $16 \%$ of NS men, $6 \%$ of S) experienced chronic high stress; and $20 \%$ had no preventive dental care. Among those aged $45-79,32 \%$ of women and $21 \%$ of men were unpaid caregivers for an ill or disabled relative. Only $36 \%$ (33\% of NS, $48 \%$ of S) felt very satisfied with their life. About $14 \%$ do not believe health habits/lifestyle can greatly affect their health, and $16 \%$ do not believe the same for emotional troubles/stress. Ability to use the internet was high ( $98 \%$ of NS, $84 \%$ of S) but lower among lower-income adults. Significant racial/ethnic disparities were observed for some SDOHs.

Conclusion: SDOHs are prevalent in socioeconomically diverse (non-safety net) health plan memberships.
P12.02

Racial/Ethnic Disparities in Completion of the Routine Immunization Series by Age Two

James Nordin, ${ }^{1}$ Gabriela Vazquez-Benitez, ${ }^{1}$ Elyse Kharbanda ${ }^{1}$

\section{${ }^{1}$ HealthPartners Institute, Minneapolis, $M N$}

Background: Immunization is the most effective clinical preventive service for children. While a large body of literature exists on racial disparities in health, data on immunization disparities is limited and the results are varied. Goals of this study were to evaluate whether racial/ ethnic disparities in immunization completion rates by age 2 years remain after adjusting for other factors, and to explore potential system-level factors that may be contributing to the disparity.

Methods: This was a retrospective cohort study that included clinic patients who had their second birthday between January 1, 2009, and December 31, 2013, and who had at least one primary care visit to a clinic within the 6 months before their second birthday. A full cohort and a mother-matched nested cohort with more granular race data and additional factors were both analyzed. Vaccines were identified using the electronic health record. The primary outcome was completion of the infant immunization schedule by 2 years of age. Logistic regression was used to evaluate associations.

Results: The full cohort included 23,601 subjects, of which 10,125 (43\%) were nonwhite. At age 24 months, the nonwhite patients had a completion rate of $75 \%$ and the white patients had a completion rate of $79 \%$. After adjustment, nonwhite patients were less likely to be vaccinated (odds ratio: $0.89,95 \%$ confidence interval: 0.83-0.94). Rates were significantly improved by more clinic visits, well-child visits and white race. The nested mother-matched cohort included 9,066 subjects, of which 3,324 (37\%) were nonwhite. The completion rates varied from $76.9 \%$ for East African origin to $83.3 \%$ for white. After adjustment of other factors, only in the East African origin group (primarily Somali) was the rate significantly lower than the white group $(0.53,0.4-0.70)$. Other characteristics that were associated with improved completion were greater number of visits, having a wellchild visit and more total months with insurance.

Conclusion: We observed modest disparities in vaccine series completion by age 2 . These results suggest two strategies for decreasing the disparity in completion: outreach to schedule well-child visits, and structural changes in insurance to make continuous coverage more likely. 


\section{INNOVATION DISSEMINATION AND IMPLEMENTATION}

P13.01

\section{Lessons Learned in Developing and Implementing the ED-PACT Tool: An Innovation Supporting Communication of Care Needs After Emergency Department Visits}

Kristina M. Cordasco, ${ }^{1}$ Hemen N. Saifu, ${ }^{1}$ Mana Khafaf, ${ }^{1}$ Jonie J. Hsiao, ${ }^{1}$ Brian J. Doyle, ${ }^{1}$ David A. Ganz ${ }^{1}$

${ }^{1}$ VA Greater Los Angeles Healthcare System, Los Angeles, CA

Background: Communication failures between providers represent a threat to patient safety. Despite the importance of timely receipt of recommended post-emergency department (ED) care, up to two-thirds of patients discharged from EDs do not receive recommended post-ED care. The ED-PACT tool uses the electronic health record to send messages from Veterans Health Administration ED providers to nurse care managers of patients' VA Patient-Aligned Care Team (PACT) when patients are discharged home from the ED with an urgent or specific follow-up need. We developed, piloted and formatively evaluated the spread of this tool at VA Greater Los Angeles Healthcare System (VAGLAHS).

Methods: Before implementation, we assessed readiness to participate in the innovation with leadership interviews and nurse care manager questionnaires. During deployment, we used audit and feedback to monitor adherence with correct use of the tool. We logged all user feedback, tracked all failures (ie, PACT nurse not acting on message) and their causes, and used run charts to assess for weekly variations in failures. We audited a random sample of 150 messages to capture types of care needs for which messages were sent. We interviewed leaders in three clinics about perceptions of usability and value, and implementation facilitators and barriers.

Results: Between November 1, 2015, and May 31, 2016, the ED-PACT tool was used to send 1,350 messages from the VAGLAHS ED to 35 PACT teamlets across 5 primary care clinics. Care needs included: symptom recheck (55\%), care coordination $(16 \%)$, wound care $(5 \%)$, medication adjustment (5\%), laboratory recheck (5\%), radiology followup $(3 \%)$, and blood pressure recheck (3\%). On average, nurses successfully acted on $91 \%$ of messages (weekly range: $72 \%-100 \%$ ). Reasons for failure included human error, staffing shortages and technical errors. Interviews with clinic leaders revealed that the ED-PACT tool is perceived to provide substantial benefit for coordinating post-ED care by effectively communicating with patients' PACT nurses. Leaders also reported nurse training and "buy-in" facilitated implementation, whereas insufficient staff posed a barrier.

Conclusion: The ED-PACT tool facilitates communication between providers during a vulnerable care transition. Deployment of similar tools should include attention to the organizational, human and technical factors revealed by our evaluation.
P13.02

Two Models for Improving Colorectal Cancer Screening Rates in Health Plan Populations

Jennifer Coury, ${ }^{1}$ Jennifer Schneider, ${ }^{1}$ Laura-Mae Baldwin, ${ }^{2}$ Gloria Coronado, ${ }^{1}$ Beverly Green, ${ }^{3}$ Amanda Petrik, ${ }^{1}$ Keshia Bigler, ${ }^{1}$ Malaika Schwartz ${ }^{2}$

${ }^{1}$ Kaiser Permanente Center for Health Research, Portland, OR; ${ }^{2}$ University of Washington, Seattle, WA; ${ }^{3}$ Group Health Research Institute, Seattle, WA

Background: Screening decreases colorectal cancer (CRC) incidence and mortality by $30 \%-60 \%$; however, CRC screening rates remain low among minorities and low-income individuals. No available data shows the effectiveness of a direct-mail program initiated by health insurance plans that serve these populations. The BeneFIT study supports two health plans implementing a program that mails fecal immunochemical tests (FIT) to patients' homes.

Methods: We present the implementation models and decision factors about participating in BeneFIT. BeneFIT involves two health plans: one in a single state with $\sim 250,000$ enrollees, another in multiple states with several million enrollees. These health plans are using two distinct models to implement BeneFIT.

Results: One health plan is using a collaborative model. A vendor centrally mails the FIT kits and reminder letters; completed FITs are returned to the clinic, where labs are ordered. This model reduces staff burden while still enabling clinics to use their standard lab, follow-up and referral processes. Early implementation challenges have been logistical issues for smaller clinics, the need for lab vendors to provide free kits (claims pay for processing of completed FITs), and data issues with patient-clinic assignment lists. The other health plan is using a centralized model. A vendor orders and mails the FITs and conducts reminder calls; a central lab receives completed FITs and sends results to the vendor, which notifies the patient-assigned clinic. The plan uses its care coordinators to follow-up positive FITs. The model has economics of scale for administration and planbased follow-up of FIT results. Challenges to implementation have been incomplete prior CRC screening data and possible redundancy of screening. Baseline qualitative interviews with the health plans identified motivations to participate including increasing patient education, the possibility to improve screening rates and health outcomes, and the opportunity to translate a promising approach to an underserved population and formally evaluate the results. Factors that could affect future health plan decisions to maintain the direct mail approach include return rates, staff and resource requirements, and provider/patient satisfaction with the BeneFIT program.

Conclusion: Weighing the successes and challenges in these two plans will help decision makers choose between outreach strategies for CRC screening. 
P13.03

Implementing a Program to Encourage Patients to Report Breakdowns in Care: Do We Really Want to Know?

\author{
Kimberly Fisher, ${ }^{1}$ Kelly Smith, ${ }^{2}$ Thomas Gallagher, ${ }^{3}$ \\ Kathleen Mazor ${ }^{1}$
}

${ }^{1}$ Meyers Primary Care Institute / University of Massachusetts, Worcester, MA; ${ }^{2}$ MedStar Health Research Institute, Hyattsville, MD; ${ }^{3}$ University of Washington School of Medicine, Seattle, WA

Background: Patients and family members have critical insights into care experiences, including information about adverse events. However, many patients are reluctant to speak up when they suspect a breakdown in communication or medical care. We therefore designed the We Want to Know (WWTK) program to encourage patients to speak up about concerns and to enable an effective response when they do. In preparation for widespread implementation of the program in a large health care system, we sought to identify the multilevel barriers and facilitators to full-scale implementation of the program.

Methods: We conducted semi-structured interviews with hospital leaders and unit leaders at two pilot hospitals, as well as system-level leaders. Interviews focused on: 1) perceived value and potential benefits of the program, 2) barriers to implementation, and 3) strategies for overcoming barriers and achieving systemwide implementation.

Results: Preliminary analysis of the interviews revealed facilitators and barriers unique to each stakeholder level with regards to implementation of the WWTK program. Unit and hospital leaders appreciated the value of a WWTK specialist who was not part of the care team to conduct active outreach with patients and assist with problem resolution, but were concerned about resource constraints and difficulties addressing problems in the absence of a WWTK specialist. Hospital leaders found the detailed and timely feedback elicited by the WWTK specialist to be useful, but raised concerns about overlap with similar activities to elicit patient perspectives. System leaders were supportive of the program and deemed it consistent with organizational values and goals, but reiterated resource constraints especially with regards to competing initiatives. Ultimately, system leaders identified an opportunity to integrate WWTK into a current, highpriority initiative that will be implemented throughout the system.

Conclusion: Efforts to achieve systemwide implementation of a program to encourage patients to speak up about breakdowns in care and respond to the concerns raised by patients will need to consider both the benefits and barriers noted by multiple levels of stakeholders. Integrating the program into an existing initiative may overcome a major barrier to systemwide implementation; additional efforts are needed to address unit- and hospital-level barriers.
P13.04

Implementation of a New Kiosk Technology for Blood Pressure Management in a CommunityBased Primary Care Clinic

Beverly Green, ${ }^{1}$ Chai-Fung Chung, ${ }^{2}$ Sean A. Munson, ${ }^{2}$ Matthew J. Thompson, ${ }^{3}$ Laura Mae Baldwin, ${ }^{3}$ Jeffrey Kaplan, ${ }^{4}$ Randall Cline ${ }^{4}$

${ }^{1}$ Group Health Research Institute, Seattle, WA; ${ }^{2}$ Department of Human Centered Design and Engineering, University of Washington, Seattle, WA; ${ }^{3}$ Department of Family Medicine, University of Washington, Seattle, WA; ${ }^{4}$ Memorial Family of Services, Yakima, WA

Background: Asking patients to measure their own vital signs using a self-service validated blood pressure (BP) kiosk in clinic has the potential to increase patient awareness of their BP control, and free up medical assistant time for other activities. We sought to evaluate BP kiosk acceptability, usability and impact on workflow of patient BP self-measurement in a primary care clinic waiting room. Methods: A primary care clinic in eastern Washington placed two validated BP kiosks in the waiting room. Patients were asked to take their own BP and print out the results at the start of their clinic visit. Mixed-method assessments included early (2-month) and later (8-month) qualitative and quantitative descriptive assessments of kiosk implementation via meetings with clinic leaders, focus groups with clinic staff and providers, observations of kiosk users and in-clinic surveys of adult kiosk users.

Results: Of the roughly $400 \mathrm{BPs} /$ week on the kiosk, 34\% were stage 1 hypertension and $13 \%$ stage 2 or higher. Patient surveys revealed that most patients were comfortable using the BP kiosk ( $82 \%$ at 2 months and $87 \%$ at 8 months), and most thought it was accurate or more accurate than medical assistant measurements ( $81 \%$ at both time points). Initial provider concerns included accuracy, but most were confident after a discussion with the study team and use. Patients and providers saw many benefits: easier BP rechecks at the same and follow-up visits, increased patient engagement and savings of medical assistant time (1 minute 30 seconds per visit) allowing them to do other tasks. The clinic addressed early concerns such as infection (sanitary wipes), instructions (simplification) and lack of personal touch (stationing a receptionist in the waiting room). Most patients $(86 \%)$ were in favor of the clinic continuing to use the BP kiosks. Remaining challenges include kiosk privacy (possibly moving one kiosk to the examination room area) and accommodating differently sized and able patients.

Conclusion: Providers, staff and patients adapted to use of a self-service kiosk, providing opportunities for deploying saved medical assistant time for other patient-care activities. The clinic decided to keep the self-service BP kiosk after the pilot period. 
P13.05

Designing for Impact: Multidisciplinary Program to Identify Novel Interventions in Support of Recovery After Major Cancer Surgery

\section{Carmit McMullen, ${ }^{1}$ Matthew Nielsen, ${ }^{2}$ Alison Firemark, ${ }^{1}$ Denise Nakatani, ${ }^{3}$ Jean Tuthill, ${ }^{3}$ Ruth McMyn, ${ }^{3}$ Anobel Odisho, ${ }^{4}$ Michael Meyers, ${ }^{2}$ David Shibata, ${ }^{5}$ Scott Gilbert $^{6}$}

${ }^{1}$ Kaiser Permanente Center for Health Research, Portland, OR; '2University of North Carolina School of Medicine, Chapel Hill, NC; ${ }^{3}$ Kaiser Permanente Northern California, Oakland, CA; ${ }^{4}$ Department of Urology, University of Washington, Seattle, CA; ${ }^{5}$ Department of Surgery, University of Tennessee Health Science Center, Memphis, TN; ${ }^{6}$ Moffitt Cancer Center, Tampa, FL

Background: Cancer surgery is complex and is associated with significant patient morbidity, yet few resources exist to prepare patients for the complications and challenges that may follow surgery. Bladder and colorectal cancer patients who receive urinary or intestinal diversions face numerous issues that predispose them to high rates of distress and complication. Methods: After preliminary ethnographic research to identify design challenges, we held a user-centered design (UCD) workshop as a step toward developing patient- and caregiver-centered interventions to support preparation for and recovery after complex cancer surgery. We used standard UCD methods and guiding principles during the workshop, focusing on innovative and broad-scope thinking. The workshop, held in late 2014, was attended by 3 colorectal/ oncologic surgeons, 3 urologic surgeons, 5 ostomy nurses, 4 patients and caregivers, and 3 experienced UCD facilitators. Concepts that emerged from brainstorming sessions were visually represented on storyboards and voted on by the group. Highly scored concepts were further developed in small-group prototyping sessions and then presented to the entire group for review. All materials created were collected, discussions were recorded, and data were later analyzed to identify opportunities for intervention.

Results: Analysis revealed that needed educational information and resources about the surgery and recovery are generally inadequate, and that design solutions should focus on the goal of enhancing appropriate self-care during the recovery period. Four opportunity areas toward this goal were identified: 1) developing multimedia patienteducation material available prior to surgery; 2) developing personalized discharge assessment and care plans; 3 ) creating a follow-up care tool that combines educational resources, self-management assistance and symptom monitoring capabilities; and 4) developing telehealth platforms to aid patients who cannot return to formal care settings easily.

Conclusion: Diverse stakeholders concluded that research and practice improvement should prioritize the development of education and communication pathways before surgery and postoperative communication interventions, all aimed at improving appropriate self-care during recovery.
P13.06

Fidelity Evaluation of the Cardiometabolic Solution, CM-SHARE: Usage and User Acceptance

Ridhima Nerlekar, ${ }^{1}$ Jake Reimer, ${ }^{1}$ Hannah Husby, ${ }^{1}$ Alice Pressman, ${ }^{1}$ J.B. Jones ${ }^{1}$

\section{${ }^{1}$ Sutter Health, Walnut Creek, CA}

Background: For implementation of an optional-use population health management tool for providers, several factors affect adoption and contribute to continued use. We evaluated providers' fidelity to such a tool in a cohort of primary care patients with cardiometabolic (CM) conditions (diabetes, hypertension, dyslipidemia).

Methods: We implemented a CM eHealth management tool (CM-SHARE) at two primary care clinics in a large health care system in Northern California. The tool was designed to improve visit preparation and patient engagement. Quantitative data were procured from electronic health records (EHR) and from the app. Qualitative data were obtained from user-feedback interviews. CM patient encounters were matched to CM-SHARE launches, and practice characteristics were recorded for each clinician.

Results: Out of 6,184 scheduled encounters in the study period, 4,952 (80\%) were made by CM patients, for whom CM-SHARE was launched $26 \%$ of the time. Of the CM patients with encounters, $692(14 \%)$ had a CM condition as the primary diagnosis for the visit. Usage for this group was higher $(42 \%)$. Launch rates varied by practice type: scribe model (team-based) had 57\% usage; traditional model (providers working alone) had 21\% usage. Usage dropped significantly from early morning to pre-lunch ( $30 \%$ to $22 \%, \mathrm{P}<0.01)$ and from midafternoon to evening ( $28 \%$ to $20 \%, \mathrm{P}<0.01$ ). Providers reported using CMSHARE for patients with: CM history, borderline lab values, weight issues, and multiple medications or labs. Other reported factors affecting usage included: lack of reminders to launch, inability to place orders or write notes, and toggling between the EHR and CM-SHARE on one screen. Providers used graphs (42\%), trends (11\%) and progress notes $(8 \%)$ for patient education.

Conclusion: CM-SHARE was launched a quarter of the time for CM patients and more than $40 \%$ of the time for $\mathrm{CM}$-specific visits. Providers working alone were less likely to use CM-SHARE. Providers appear to fall behind on their schedule and "catch up" during the lunch break. Providers reported spending less time navigating the EHR for graphs. Simple visuals improved patient understanding and patient-provider conversation. Overall, providers were engaged in the process and enthusiastic about future releases. 
P13.07

\section{Building a Learning Health Care Organization: External Facilitation Tailors Support to the Learning Capacity of Primary Care Settings}

\author{
Michael Parchman, ${ }^{1}$ Clarissa Hsu, ${ }^{1}$ L.J. Fagnan, ${ }^{2}$ \\ Nicole van Borkulo, ${ }^{1}$ Leah Tuzzio ${ }^{1}$ \\ ${ }^{1}$ Group Health Research Institute, Seattle, WA; ${ }^{2}$ Oregon \\ Health Science University, Portland, OR
}

Background: Organizational theory describes facilitation as an absorptive capacity meta-routine that enhances an organization's ability to acquire and apply new knowledge to improve performance. Tailoring of the facilitation to the individual practice setting has been hypothesized as one mechanism that explains its effectiveness. Herein we describe tailoring of conversations by facilitators to the absorptive capacity of primary care practices for the purpose of expanding their quality improvement (QI) ability.

Methods: The Healthy Hearts Northwest study is testing strategies to build QI ability within 203 independent smaller primary care practices across Washington, Oregon and Idaho. Practice facilitators conduct quarterly in-person visits and monthly phone calls in between visits. During the initial visit, the facilitator meets with the practice team to develop consensus responses to 20 questions about QI capacity in 7 domains: embedding clinical evidence, using data, establishing a QI process, population management, defining team roles, self-management support, and community resources. Facilitators document the number and type of topics discussed after each contact with the practice. We examined the association between QICA results and the number and type of topics discussed. We also drew on notes generated by the facilitators after every encounter with a practice and focus group data from facilitators.

Results: The mean QICA score was 6.52 (standard deviation [SD]: 1.45, range: 3.3-10.8). Total topics discussed ranged from 0 to 26 with a mean of 5.39 (SD: 5.08) Total QICA score was correlated with number of topics discussed $(\mathrm{P}<0.01)$. The number of health information technology topics discussed correlated with practice capacity to use data; the number of QI topics discussed correlated with capacity regarding team roles and functions. Comments from facilitators suggest that the QICA discussion was valuable by providing consensus on current state of QI within the practice, prioritizing next steps to build QI capacity, creating buy-in among team members and developing a common vision.

Conclusion: External facilitators tailor their level of effort and the content discussed with individual practices to their current level of absorptive/learning capacity. Tailoring support explains how external facilitation can build learning capacity for improvement within a health care organization.
P13.08

Improving Reliability of Scheduling Post-Acute Follow-Up Care, Implementing One Element of Project RED

\author{
Alice Pressman, ${ }^{1}$ J.B. Jones, ${ }^{1}$ Mani Aravind ${ }^{2}$ \\ ${ }^{1}$ Research, Development and Dissemination, Sutter Health, \\ Walnut Creek, CA; ${ }^{2}$ California Pacific Medical Center, \\ Sutter Health, San Francisco, CA
}

Background: The New England Journal of Medicine reports that 1 in 5 discharged Medicare patients is readmitted to a hospital within 30 days. Readmissions are costly to hospitals and are a major disruption to a patient's life, creating financial and emotional strain. Project Re-Engineered Discharge (RED), a nationally recognized program to reduce preventable hospital readmissions, was implemented at Sutter Health's California Pacific Medical Center (CPMC) beginning in late 2013. The most challenging of the 12 components of the intervention is the coordination and timely completion of follow-up care with a primary care physician after hospital discharge.

Methods: To ensure that each patient going home from the hospital would have a post-acute care appointment scheduled, we developed Discharge Planner, a web-based software application to support the required multistep, multidisciplinary workflow. We then piloted the tool in a single unit at CPMC. We measured fidelity by monitoring whether case managers were launching the application and recorded the proportion of patients with appointments scheduled at the time of discharge. We used patient electronic health records to measure the proportion of patients who attended follow-up appointments postdischarge. Finally, we used provider surveys to determine user acceptability.

Results: The app was opened an average of 40 times/day for a single hospital unit during business hours and $<5$ times/day on weekends. Follow-up appointment scheduling increased from $<20 \%$ during the 4 months "pre-go-live" to $>50 \%$ during the 5-month "post-go-live" period. Only 20\%-30\% of patients kept their scheduled appointments throughout the study period, with no change during the "post-go-live" period. User acceptance scores were highly favorable (on a scale from 1 to 100 , average scores were 73 overall and 87 among those case managers who rely most heavily on the application).

Conclusion: The application was highly successful at accomplishing its primary goal, scheduling follow-up appointments, and it has been accepted into the workflow. However, patients still do not appear to be keeping their follow-up appointments. Our next step is to uncover strategies to better measure kept appointments and to identify factors that prevent patients from keeping their appointments. 
P13.09

Geisinger's Use of Clinical CarePaths: Impact of a Psoriasis CarePath on Process, Clinical, and Economic Outcomes

\author{
Rebecca Pulk, ${ }^{1}$ Laney Jones, ${ }^{1}$ Joseph Chronowski, ${ }^{2}$ \\ Howard Pride, ${ }^{3}$ Eric Wright, ${ }^{1}$ Michael Evans ${ }^{1}$
}

${ }^{1}$ Center for Pharmacy Innovation and Outcomes, Geisinger Health System, Danville, PA; ${ }^{2}$ Geisinger Institute for Advanced Application, Danville, PA; ${ }^{3}$ Geisinger Dermatology, Danville, PA

Background: CarePaths are evidence-based integrative care processes that seek to treat patients with complex diseases in a standardized manner. Herein we describe the development and implementation of the CarePath for psoriasis and present preliminary economic and clinical outcomes of this streamlined process.

Methods: The CarePath for psoriasis was developed through a five-step process involving population identification, care algorithm development (synchronized to coverage determinations), IT development, patientfamily engagement, and outcome monitoring utilizing input from our integrative delivery network of patients, providers and payers. Over the course of 12 months, the multidisciplinary team developed standardized data elements within the electronic health record (Epic) and a psoriasis-specific performance dashboard to ensure consistent population targeting, outcome monitoring and provider compliance tracking. Monitoring within the build allows for evaluations of the adoption of the CarePath including: body surface area tracking and completion, inclusion of psoriasis on the patient's problem list, utilization of nonpharmacological options (eg, light therapy), and drug therapies used. A simple cost-avoidance model of selecting light therapy over alternative biologic therapy was employed to calculate savings.

Results: Adoption of the psoriasis CarePath has steadily increased since its launch in July 2015. Inclusion of psoriasis in the problem list of affected patients steadily increased in the 12 months post-CarePath launch. Tracking of body surface area measurements increased from $41.7 \%$ to $76.3 \%$ over the same time period. A total of 72 patients initiated light therapy since CarePath implementation, 61 of whom were biologic candidates and 11 who switched from biologics. With an estimated 6-year single patient cost of $\$ 2,200$ for one ultraviolet light or $\$ 294,000$ for formulary biologic alternatives, the psoriasis CarePath is estimated to save the health system $\$ 21,009,600$ ( $\$ 3,501,600$ /year).

Conclusion: Through the psoriasis CarePath, we have been able to standardize the care of patients across Geisinger Health System by providing patient-focused, evidence-based care at substantial cost savings. Lessons gleaned through the early success of the psoriasis CarePath are being applied to CarePath construction for rheumatoid arthritis, heart failure, pulmonary hypertension and other diseases.
P13.10

Using Novel Multimodal Reminders for a Direct-Mail Fecal Testing Program: Findings From STOP CRC

Jennifer Rivelli, ${ }^{1}$ Gloria Coronado, ${ }^{1}$ Amanda Petrik, ${ }^{1}$ Sara Barker, ${ }^{2}$ Emily Topalanchik, ${ }^{2}$ Erin Keast, ${ }^{1}$ Keshia Bigler, ${ }^{1}$ Ricardo Jimenez ${ }^{2}$

${ }^{1}$ Kaiser Permanente Center for Health Research, Portland, OR; ${ }^{2}$ Sea Mar Community Health Centers, Seattle, WA

Background: Evidence shows that reminders can improve rates of patient adherence to completion of cancer screening tests. Less is known about the efficacy of reminders in relation to direct-mail fecal testing programs. As part of the Strategies and Opportunities to STOP Colon Cancer in Priority Populations (STOP CRC) study, we sought to compare the effectiveness of multimodal reminders for a direct-mail fecal testing program.

Methods: We tested screening reminders in four clinics at Sea Mar, a community health center in Washington State. Adults aged 50-75 years who had a primary care visit in the previous year and were not up to date with colorectal cancer screening received an informational letter and fecal immunochemical test (FIT) kit by mail. Patients who did not return their FIT kits within 3 weeks were sent a message through the patient portal or randomized to receive: 1) a reminder letter; 2) three automated phone calls; 3 ) three text messages; 4) a live phone call; 5) a text message and a live phone call; 6) a reminder letter and a live phone call; or 7) an automated and live phone call. We recorded rates of screening completion and rates of patients reached by reminder.

Results: On initial data analysis, reach among combined strategies was highest for automated and live phone call $(27 \%)$. For single-modal reminder strategies, reach was highest for live phone call (30\%) and lowest for text message $(13 \%)$. We are currently receiving data on the last clinics. Final results will be presented in the poster.

Conclusion: To date, in the federally qualified health center setting, automated and live phone call reminders to a directmail fecal testing program substantially increase colorectal cancer screening rates.

\section{MENTAL HEALTH, ALCOHOL AND SUBSTANCE USEIABUSE}

\section{P14.01 \\ Using Natural Language Processing to Enable Quality Improvement and Future Research for Patients at Risk of Suicide}

Jennifer M. Boggs, ${ }^{1}$ J. David Powers, ${ }^{1}$ LeeAnn M. Rohm, ${ }^{1}$ Steve Hochberg, ${ }^{1}$ Arne Beck ${ }^{1}$

${ }^{1}$ Institute for Health Research, Kaiser Permanente Colorado, Denver, $C O$ 
Background: Policy research demonstrates that lethal means restriction is an effective strategy for preventing suicide. This evidence has informed national suicide prevention recommendations for medical/behavioral providers to assess patient access to lethal means. The clinical uptake and effectiveness of this practice is unknown. Behavioral health providers document means assessment and restriction counseling in progress notes, but not in structured form for easy extraction. We developed a natural language processing (NLP) query to identify lethal means assessment and restriction counseling within semi-structured clinical notes.

Methods: A total cohort of 11,259 adult Kaiser Permanente Colorado patients who had either a suicide attempt or positive suicide item on the Patient Health Questionnaire (PHQ-9) depression measure from 2010 to 2015 was queried. To identify key terms for the NLP query, we used manual chart review and text mining. All encounters for 1 month following index event (attempt or ideation) were reviewed for 100 patients. Using manual chart review as the gold standard, supervised text mining identified terminology indicating evidence of means documentation. Text mining, clinical consultation and chart review results informed query criteria that were implemented using open source NLTK Python package for NLP. The query was tested and modified throughout three iterations. Negative/ positive hits were analyzed on stratified random samples of 40 charts/round. The final query was validated using manual review on a hold-out sample of 200 charts.

Results: We will present a description of the final query, including terms/phrases used, qualifiers, stop words and synonyms. Sensitivity, specificity and positive/negative predictive values for assessment of lethal means and means restriction counseling will be reported on the development and hold-out samples from the final query.

Conclusion: The query will allow us to identify the proportion of high-risk patients who receive recommended assessment of lethal means following suicide attempts/ ideation. This query could be used for operational quality improvement or to inform future research on the effectiveness of lethal means assessment/counseling to restrict means in preventing suicide outcomes. Detailed specifications on the methods used to create this NLP query will be made available as a resource for other systems.

\section{P14.02}

Alcohol Misuse Among Formerly Deployed U.S. Service Members Seen in Non-VA Facilities: Results From the Veterans' Cohort Study

Joseph Boscarino, ${ }^{1}$ Stuart Hoffman, ${ }^{1}$ Thomas Urosevich, ${ }^{1} \mathrm{H}$. Lester Kirchner, ${ }^{1}$ Johanna Hyacinthe, ${ }^{1}$ Richard Adams, ${ }^{2}$ Charles Figley ${ }^{3}$

${ }^{1}$ Geisinger Health System, Danville, PA; ${ }^{2}$ Kent State University, Kent, OH; ${ }^{3}$ Tulane University, New Orleans, LA
Background: Since reports suggest that alcohol misuse is a health problem among U.S. military personnel, our objective was to assess the prevalence of alcohol-use disorders among formerly deployed service members seen at non-VA health care facilities. Because research also suggested higher alcohol abuse among Vietnam veterans, our hypothesis was that Vietnam veterans would have a higher prevalence of alcohol misuse than deployed veterans from the other service eras.

Methods: We surveyed a random sample of veterans who were patients in a large non-VA multihospital system located in central and northeastern Pennsylvania to assess their mental health and substance use. The study included veterans from four service eras: Vietnam, Gulf War, Global War on Terror, and other veterans.

Results: Of 1,289 veterans surveyed (response rate: $\sim 60 \%$ ), $53.6 \%$ were from the Vietnam era, $95.0 \%$ were male, $54.5 \%$ were $65+$ years old, $95.7 \%$ were white race, and $26.9 \%$ were recent National Guard or Reserve veterans. Based on the AUDIT-C and CAGE instruments, the prevalence of alcohol misuse was $27.3 \%$ and $14.1 \%$, respectively, compared to only $8.7 \%$ for current posttraumatic stress disorder and $8.8 \%$ for current depression. Altogether, $25.8 \%$ reported using alcohol to cope postdeployment, and $21.0 \%$ reported heavy drinking in the past year. Bivariate analyses indicated that alcohol misuse was more common among those who were older, Vietnam veterans, higher-income veterans, and those who had a history of cigarette smoking $(\mathrm{P}<0.05$ for all). However, multivariable analyses that adjusted for gender, education, combat exposure, life stressors and social support found no significant differences for alcohol misuse or abuse by the different veteran groups. The best predictors of current alcohol misuse on multivariable analyses were having used alcohol to cope postdeployment (odds ratio: $2.99, \mathrm{P}<0.001$ ) and younger age (odds ratio: 0.97, $\mathrm{P}<0.001$ ). Conclusion: Our analyses suggest that while deployed Vietnam service members had a higher prevalence of alcohol misuse, when the data were adjusted for demographic factors and potential confounders, there were no significant differences between the veteran groups. Further research that examines the high prevalence of alcohol misuse among veterans and the adverse impact of using of alcohol to cope postdeployment is planned.

\section{P14.03}

Differences in Adult PHQ9 Administration at KP Phillip Crawford, ${ }^{1}$ Frances Lynch, ${ }^{1}$ Greg Clarke,${ }^{1}$ Greg Simon ${ }^{2}$

${ }^{1}$ Kaiser Permanente Center for Health Research, Portland, OR; ${ }^{2}$ Group Health Research Institute, Seattle, WA

Background: The Patient Health Questionnaire (PHQ-9) is a depression screener that scores each of the nine DSMIV depression criteria from the original PRIME-MD. With 
the increased administration and electronic capture of data from the PHQ-9 instrument, it has added research value to its existing usefulness as a clinical evaluation tool. Recent inclusion in Health Care Systems Research Network's virtual data warehouse (VDW) means that researchers can look at PHQ-9 data in the context of other important patient health and demographic factors.

Methods: We interrogated the Kaiser Permanente Northwest (KPNW) VDW for demographic, diagnosis, enrollment, and PHQ-9 and U.S. Census data for the years 2014-2015. Age, gender and race/ethnicity were combined with Census education and income, PHQ-9 dates and ICD-9/10 depression diagnoses. Within the sampling frame of all adults aged 18-90 years with any KPNW enrollment in 2014 and 2015, we compared four groups: among patients with a PHQ-9 screening over the study years, those (a) with, and (b) without one or more clinical depression diagnoses; and among patients without a PHQ-9 screening, those (c) with, and (d) without one or more depression diagnoses.

Results: Patients completing the PHQ-9 differed in several ways from adults who did not complete the PHQ-9. The PHQ-9 group was older (mean age: 49.7 vs 45.9 years) and more often female $(64.6 \%$ vs $49.4 \%)$ and non-Hispanic white $(82.1 \%$ vs $66.7 \%)$. A notably lower percentage of PHQ-9 screened patients were non-Hispanic Asian (2.4\% vs $4.7 \%$ with no screening). As expected, the PHQ-9 group was demographically similar to the group with the depression diagnoses, with some minor differences on gender. Patients with a depression diagnosis and a PHQ-9 administration were slightly more often female compared to those without a PHQ-9 (69.9\% vs 66.9\%). Census comparisons indicate that patients with a depression diagnosis and a PHQ-9 lived in areas with slightly lower educational attainment versus patients with a depression diagnosis only $(28 \%$ of adults in their Census area had a bachelor's degree vs 31\% without, respectively). No differences were observed on income.

Conclusion: The PHQ-9 is differentially administered among adult patients at KPNW. But the administration does follow the demographic spectrum of depression.

\section{P14.04}

\section{Opioid Prescribing During Pregnancy: Eight-Year Secular Trends at HealthPartners Medical Group}

Thomas E. Elliott, ${ }^{1}$ Caitlin K. Frail, ${ }^{2}$ Pamala A. Pawloski, ${ }^{1}$ Avis J. Thomas, ${ }^{1}$ Ann M. Werner, ${ }^{1}$ Rebecca C. Rossom ${ }^{1}$

${ }^{1}$ HealthPartners Institute, Bloomington, $M N ;{ }^{2}$ College of Pharmacy, University of Minnesota, Minneapolis, $M N$

Background: Nationally, opioid prescribing for chronic noncancer pain has increased significantly over the last 20 years. Recently published evidence suggests that $20 \%$ to $40 \%$ of women received opioid prescriptions of varying doses and durations during pregnancy, which may cause increased risks of harm to mother and fetus. The purpose of this retrospective observational study was to determine the prevalence of opioid prescribing 3 months before pregnancy, each trimester of pregnancy and 3 months postpartum, as well as the secular utilization trend over an 8-year period, among member-patients at HealthPartners Medical Group (HPMG).

Methods: All pregnant member-patients of HPMG who delivered a live birth from 2006 to 2014 and had continuous pharmacy benefits beginning 3 months prior to their estimated pregnancy start through 3 months postpartum were included. Demographic, clinical, pharmaceutical and provider variables of interest were identified and described. Significant opioid prescribing during pregnancy was defined as more than 5 days' supply prescribed in any 3-month period, excluding the 2 -week postpartum period. Time trends for 2006-2014 were examined using linear regression.

Results: Of 11,565 pregnancies during the study period, significant opioid prescribing during 3 months before pregnancy, pregnancy or 3 months postpartum were observed in $862(7.5 \%)$ pregnancies (816 unique women). A total of 454 (3.9\%) pregnant women received significant opioid prescriptions during one or more trimesters of their pregnancy. From 2006 to 2014, the rate of significant opioid prescribing during each trimester of pregnancy and 3 months before and after pregnancy decreased $-0.2 \%$ per year.

Conclusion: Significant opioid prescribing during the three trimesters of pregnancy as well as 3 months before and after pregnancy for member-patients of HPMG was significantly lower than reported studies from other populations and locations across the United States over the last 15 years. Furthermore, significant opioid prescribing was trending downward slightly over time, rather than rising as reported elsewhere. Explanatory factors for these findings should be explored.

\section{P14.05 \\ Defining Depression Cohorts Using the Electronic Health Record: ICD-9 Codes Versus Medication Orders}

\section{Wendy Marie Ingram, ${ }^{1}$ Sharon Larson ${ }^{1}$ \\ ${ }^{1}$ Geisinger Health System, Danville, PA}

Background: Electronic health records (EHR) allow health care researchers to conduct unprecedented largescale studies on diseases, treatments and health care system utilization. EHR studies are limited by the quality of the data set available. Careful consideration must be given to how to define patient cohorts. One approach aimed at limiting the number of nonclinically relevant patients included in a cohort is rigid inclusion criteria. With rigid inclusion criteria, however, we run the risk of excluding those with clinical features who are receiving treatment but do not meet these criteria. They may not meet these criteria due 
to patient or provider bias against including certain features like ICD-9 codes in their health record, or perhaps there are administrative data sequestration protocols inherent in the system, barring researcher access to pertinent patient information. This may be the case with certain psychiatric conditions.

Methods: We have compared two methods of defining a cohort of depressed patients using information in the EHR.

Results: We show that either using ICD-9 codes for depression or medication orders for antidepressants results in exclusion of potentially clinically relevant patients in both cases. We also show that both of these methods result in cohorts with highly correlated clinical features such as emergency department usage and primary discharge diagnosis codes, outpatient clinic visitation frequency and inpatient discharge diagnosis codes.

Conclusion: For the case of defining a cohort to study depression, less rigid electronic phenotypes may better capture patients who are receiving some sort of treatment for depression.

\section{P14.06}

\section{Treating Behavioral Health Conditions: What Worries Pediatric Residents?}

Ilene Ladd, ${ }^{1}$ Paul Kettlewell, ${ }^{1}$ Jeffrey Shahidullah, ${ }^{2}$ Kathy Dehart, ${ }^{1}$ Tyler Bogaczyck, ${ }^{1}$ Sharon Larson ${ }^{1}$

${ }^{1}$ Geisinger Health System, Danville, PA; ${ }^{2}$ Rutgers University, New Brunswick, NJ

Background: Changes in the delivery of health care have increased attention to behavioral health care. Particularly, there is an emphasis on improving the delivery of behavioral health care through integrated care delivery systems. Unfortunately, many medical students emerge from their medical school experience with little more than a brief exposure, often a 1-month rotation, in a psychiatric setting. As residents arrive for their first and subsequent years of training, formal exposure to behavioral training often does not occur. Pediatric residents frequently begin their residency in "continuity clinic," where they are providing primary behavioral care to children, adolescents and their parents. This care often involves providing guidance about behavioral health issues. Residents are often "flying by the seat of their pants" in this particular care area because there has not been formal training. This study represents a qualitative study of Year 1 residents early in their first year of residency and as they transition into Year 2 of their pediatric residency. It is intended to document their concerns, knowledge and attitudes about providing behavioral health care. Residents will be followed in this project for 3 years as a program of formal didactic education. Integration of clinical psychologists, curbside consults and shared visits is implemented to address gaps in behavioral health experience and knowledge.
Methods: Residents participated in focus groups during years 1 and 2 of their residency. Discussion focused on knowledge gaps, concerns and successes in delivery of behavioral health care within their general pediatric practice.

Results: Several key themes emerged including time management, struggles with establishing rapport with patients, knowing referral sources and protocols, comfort level diagnosing but not knowing how to treat a variety of conditions, difficulties in establishing communication and relationships with adults and dysfunctional families, making mistakes that result in children dying, and the relative importance of behavioral health.

Conclusion: Changes in residency training to improve skills in behavioral health treatment may be warranted.

\section{P14.07 \\ Population-Based Outreach Versus Usual Care to Prevent Suicide Attempt: Study Protocol for a Randomized Clinical Trial}

Rebecca C. Rossom, ${ }^{1}$ Greg Simon, ${ }^{2}$ Arne Beck, ${ }^{3}$ Julie Richards, ${ }^{2}$ Beth Kirlin, ${ }^{2}$ Deborah King, ${ }^{2}$ Lisa Shulman, ${ }^{2}$ Evette Ludman, ${ }^{2}$ Rob Penfold, ${ }^{2}$ Susan Shortreed, ${ }^{2}$ Ursula Whiteside ${ }^{2}$

${ }^{1}$ HealthPartners Institute, Minneapolis, MN; ${ }^{2}$ Group Health Research Institute, Seattle, WA; ${ }^{3}$ Kaiser Permanente Colorado, Denver, $C O$

Background: Suicide is the 10th leading cause of death. PHQ-9 item \#9 (which asks about suicidal thoughts) identifies those at risk of suicide attempt/death. Patients with scores of 2 or 3 on item \#9 show a sustained increase in risk, with a cumulative hazard near $4 \%$ over 12 months. Methods: Outpatients who score a 2 or 3 on item $\# 9$ of the PHQ-9 are identified using electronic health record (EHR) data at three Mental Health Research Network sites: Group Health Cooperative, HealthPartners and Kaiser Permanente Colorado. Using a modified Zelen design, patients are automatically assigned $1: 1: 1$ to continue in usual care (ie, no contact) or to be offered one of two populationbased prevention programs meant to supplement usual care: 1) Care Management (systematic outreach to assess risk, EHR-based tools for risk-based pathways, and care management to facilitate and monitor recommended follow-up care); or 2) Skills Training (interactive online training in dialectical behavioral therapy skills supported by reminder and reinforcement messages). Randomization automatically occurs within each site's sampling computer program, stratified by item $\# 9$ score. A computer-generated concealed allocation table provides randomly generated assignments in block sizes of either 6 or 9 . The multisite interventions are embedded in the EHR. Online patientprovider secure messaging via the EHR patient portal is used for patient invitation and outreach as well as 
administration of suicide risk questionnaires. Secure provider-to-provider messaging is used to communicate with primary care and mental health providers. Population management and reporting tools are used to apply followup algorithms and deliver recommendations to care managers regarding outreach and follow-up. Nonfatal and fatal suicide attempts are identified using state vital statistics data and diagnoses of self-inflicted injury from EHR and claim records. Primary evaluation will compare risk of first suicide attempt over the 18 months following randomization. Groups will be compared according to initial treatment assignment, regardless of level of participation in either intervention.

Results: To date, 4,869 outpatients out of a planned 18,000 have been randomized across the three sites.

Conclusion: Our experience thus far illustrates the promise and challenges of implementing multisite clinical trial recruitment and intervention delivery in EHR systems.

\section{P14.08}

\section{Adolescent SBIRT in Pediatric Primary Care: Patient Outcomes From a Randomized Trial in an Integrated Health Care System}

\author{
Stacy Sterling, ${ }^{1}$ Andrea Kline-Simon, ${ }^{1}$ Ashley Jones, ${ }^{1}$
} Anna Wong, ${ }^{2}$ Derek Satre, ${ }^{1}$ Constance Weisner ${ }^{1}$

${ }^{1}$ Kaiser Permanente Division of Research, Oakland, CA; ${ }^{2}$ The Permanente Medical Group, Oakland, CA

Background: Many adolescents in need of specialty treatment for substance use and mental health problems never access care. Screening, brief intervention and referral to treatment (SBIRT) is a promising approach to early identification and intervention for adolescent substance use. We describe patient outcomes from a trial of different modalities of SBIRT for adolescents in primary care.

Methods: We randomized pediatricians $(\mathrm{N}=52)$ in a pediatrics clinic to three study arms: 1) pediatrician-only, in which pediatricians were trained to deliver SBIRT; 2) embedded behavioral health clinician (BHC), in which pediatricians referred adolescents who endorsed risk factors (substance use or mood symptoms) to a BHC; and 3) usual care (UC). We used electronic health record (EHR) data to examine risk factors, CRAFFT scores, treatment referral, initiation and engagement.

Results: 1,871 patients were eligible for assessments, brief interventions and referrals. Differences in outcomes between the index and the next well visit within 2 years were examined across the three arms. The odds of risk factor endorsement decreased over time for all patients (adjusted odds ratio [aOR]: 0.07; 95\% confidence interval [CI]: 0.05-0.11); the embedded-BHC arm had significantly lower odds of risk factor endorsement compared to UC (aOR: 0.65; 95\% CI: 0.43-0.97); there were no differences between UC and the physician-only arm. There were no differences in CRAFFT scores over time or between intervention arms. The embedded-BHC arm had fewer referrals to specialty treatment compared to the other study arms; referral rates between the physician-only and UC arms did not differ. Among those referred, the physicianonly arm had significantly lower odds of treatment initiation compared with UC (aOR: 0.53; 95\% CI: 0.28-0.99) and the embedded-BHC arm (aOR: 0.25 ; 95\% CI: 0.12-0.49); no differences were found between UC and embeddedBHC. There were no differences in treatment engagement (at least 2 visits within 30 days) across the arms.

Conclusion: Patients in the embedded-BHC arm reported lower behavioral health risk at subsequent screenings, and the embedded-BHC intervention arm was more effective at facilitating treatment initiation for those adolescents needing specialty behavioral health services.

P14.10 Health Care Service Utilization Among Anxious
and Nonanxious Youth

V. Robin Weersing, ${ }^{1}$ Argero Zerr Zerr, ${ }^{2}$ John Dickerson, ${ }^{3}$ Frances Lynch, ${ }^{3}$ Phillip Crawford, ${ }^{3}$ Kate Conover ${ }^{1}$

${ }^{1} S D S U-U C S D$ Joint Doctoral Program in Clinical Psychology, San Diego, CA; ' 2 San Diego State University, San Diego, CA; ${ }^{3}$ Kaiser Permanente Northwest, Portland, OR

Background: Anxiety disorders are the most common mental health problem, impacting $15 \%$ to $20 \%$ of youth at any given time. Despite high prevalence, little is known about the type of health care services utilized by anxious youth, impeding public health efforts to improve access to and quality of care. To address this need, the current study will directly compare service utilization in a large sample of anxious and nonanxious youth patients enrolled within Mental Health Research Network (MHRN).

Methods: Preliminary analyses were conducted using a sample of 17,929 youths (ages 4 to 17 years) from Kaiser Permanente Northwest (KPNW). Anxiety diagnoses were derived from ICD-9 codes and service use from procedure codes during 2013-2014. Analyses were completed using a match-control design, in which anxious youth were matched with their nonanxious peers using sociodemographic indices. Results: Results from the KPNW site showed that anxious youth were significantly more likely to receive care than nonanxious matched controls in pediatrics (odds ratio [OR]: 2.28; $\mathrm{P}<0.001$ ), family medicine (OR: 1.36; $\mathrm{P}<0.001$ ), emergency departments (OR: 2.23; $\mathrm{P}<0.001$ ), and urgent care (OR: 1.66; $\mathrm{P}<0.001)$. Anxious youth also were more likely to receive services in specialty care settings such as outpatient mental health (OR: 17.34; $\mathrm{P}<0.001$ ), inpatient mental health (OR: 16.56; $\mathrm{P}<0.001$ ), neurology (OR: 3.71; $\mathrm{P}<0.001)$ and cardiology (OR: 2.85; $\mathrm{P}<0.001)$. Overall, anxious youths in this insured sample were high utilizers of services, including increased use of high-cost services. 
Conclusion: Final analyses will integrate data from an additional three MHRN sites using innovative statistical methods designed to pool and analyze de-identified services data across multiple sites. The present study will be one of the first to examine health services use for a large and diverse sample of anxious youth across several different health care settings and systems. Findings from this study will provide unique and critical information about the availability and type of care currently utilized by anxious youth. Results may be useful in guiding efforts to most efficiently intervene with this widely prevalent and highly impairing condition.

\section{PATIENT, CLINICIAN, AND HEALTH SYSTEM ENGAGEMENT}

\section{P15.01}

\section{Expanding the VA Women's Health Practice-Based Research Network: Increasing Capacity for Equitable Representation of Women in VA Research}

Diane Carney, ${ }^{1}$ Susan Frayne, ${ }^{1,5}$ Ruth Klap, ${ }^{2}$ Lori Bastian, ${ }^{3}$ Bevanne Bean-Mayberry, ${ }^{2}$ Anne Sadler, ${ }^{4}$ Alyssa Pomernacki, ${ }^{1}$ Ciaran Phibbs, ${ }^{1,5}$ Fay Saechao, ${ }^{1}$ Vidhya Balasubramanian, ${ }^{5}$ Yasmin Romodan, ${ }^{1}$ Elizabeth Yano ${ }^{2}$

${ }^{1}$ VA Palo Alto Health Care System, Palo Alto, CA; ${ }^{2} V A$ Greater Los Angeles Healthcare System / University of California, Los Angeles, Los Angeles, CA, ${ }^{3} V A$ Connecticut Healthcare System / Yale University, West Haven, CT; ${ }^{4}$ Iowa City VA Health Care System / University of Iowa, Iowa City, IA; ${ }^{5}$ Stanford University, Stanford, CA

Background: The Veterans Affairs (VA) Women's Health Practice-Based Research Network (WH-PBRN) facilitates multisite women's health research through a network of partnered VA facilities and fosters bidirectional partnership of clinicians and researchers striving to improve the health and health care of women veterans. Initial WH-PBRN development was informed by pilot studies conducted at four inaugural sites. The WH-PBRN then expanded to 37 VA sites in 2012. To further grow into a more diverse network, a call for site applications went out March 2015; WH-PBRN expanded to 60 sites in June 2015. As a program evaluation activity, we examined representativeness of WHPBRN sites (relative to VA sites nationally) before and after expansion.

Methods: WH-PBRN site applications included questions about facility characteristics, including items indicating level of local research support such as presence of an affiliated Health Services Research and Development (HSR\&D) center at the facility. We coupled those data with aggregated, site-level, national VA administrative data from fiscal year 2014 describing characteristics of women veteran patients using each site.
Results: From pre- to post-expansion, the network grew from 37 sites, representing 146,706 women veteran outpatients, to 60 sites, representing more than half of all WV outpatients (220,465 of 391,062 women veterans nationally). Expansion successfully increased representation of diverse populations. For example, across sites, number of women veterans from a racial/ethnic minority group increased from $38 \%$ pre to $40 \%$ post. Member sites together are fairly representative of Veterans Health Administration (VHA) as a whole; for example, $43 \%$ of women veterans at the $60 \mathrm{WH}$-PBRN sites have age $<45$ years (vs $42 \%$ VA-wide), $22 \%$ (vs $26 \%$ ) have a rural residence, $84 \%$ (vs $91 \%$ ) used primary care, and $39 \%$ (vs $41 \%$ ) used mental health services. The main difference is that $35 \%$ have an HSR\&D center (vs 16\% VA-wide). Number of Veterans Integrated Service Networks represented increased from 17 pre to 20 post, increasing geographic heterogeneity.

Conclusion: WH-PBRN represents a large and geographically diverse nationwide network of 60 VA sites. Expansion increased the number and diversity of women veterans represented in the network. Across facilities, most women use primary care services, suggesting VHA primary care settings are an excellent venue for recruitment. Similarly, a large proportion receives mental health care: this is important since women's mental health care remains a major focus of VHA research. The expanded WHPBRN provides greater opportunity to increase equitable representation of women veterans in VA research, and to conduct health services research that examines diverse health care delivery systems.

P15.02

Organizational Learning in an Integrated Health System: Informing Operations for a Learning Health Care System

Deserae Clarke, ${ }^{1}$ Gloria Gerrity, ${ }^{2}$ Rebecca Stametz, ${ }^{1}$ Amanda Young, ${ }^{3}$ Daniel Davis ${ }^{4}$

${ }^{1}$ Geisinger Institute for Advanced Application, Danville, PA; ${ }^{2}$ Geisinger Pediatric Administration, Danville, PA; ${ }^{3}$ Geisinger Biostatistics Core, Danville, PA; ${ }^{4}$ Geisinger Bioethics, Danville, PA

Background: Since 2013 a multidisciplinary group has been working to spur Geisinger Health System's growth as a learning health care system (LHCS), using the Institute of Medicine (IOM) model as a guide. In March 2016 the group conducted a survey of staff to elicit perceptions and assess awareness of learning and aspects of the learning environment at Geisinger.

Methods: The target audience for the survey was identified by leadership and included clinical, research, administrative and information technology (IT) staff at the Geisinger Medical Center (GMC), the system's flagship hospital. The survey was sent via email using Qualtrics software, and nonresponders received two weekly reminders. 
Results: Of 559 contacted, 357 (63.9\%) staff members responded, including clinical $(\mathrm{n}=164)$, administration $(n=78)$, research $(n=23)$, IT $(n=5)$ employees and 87 others identifying with multiple areas. Most participants (62.5\%) had not previously heard of IOM's LHCS model. The majority of respondents characterized the environment at GMC $(83.2 \%)$ and in their work unit $(86.6 \%)$ as conducive to learning; however, $94.7 \%$ indicated that they were unlikely to participate in training or learning activities. The survey asked participants to assess the importance of LHCS functions to their work and to evaluate the current support they receive for these functions. Several functions were ranked as important to their work but in need of increased support: the ability to capture and view patient-reported data; the service request process; the ability to access data and use it without the assistance of a specialized data analyst; the capacity to track clinical outcomes; the ability to communicate with other providers simultaneously; and patient-centered care and patient engagement in quality improvement, innovation and research teams.

Conclusion: Although most respondents were unaware of the IOM model, the majority indicated that the GMC environment is conducive to learning. Further exploration is needed to determine why so few indicated a willingness to participate in learning activities. Areas where there is a gap between importance to work and current support offer a potential focus for future efforts to stimulate Geisinger's growth as a LHCS. Survey results were presented at a GMC symposium on learning and patient engagement, and followup activities are in process.

\section{P15.03}

\section{Stakeholder Engagement in a Patient-Activation Behavioral Intervention for Prescription Opioid Patients (ACTIVATE)}

Monique Does, ${ }^{1}$ Andrea Kline-Simon, ${ }^{1}$ Nancy Charvat Aguilar, ${ }^{1}$ Catherine Marino, ${ }^{1}$ Cynthia Campbell ${ }^{1}$

\section{${ }^{1}$ Kaiser Permanente Division of Research, Oakland, CA}

Background: The complexity of the prescription opioid epidemic has highlighted the need to address opioid use and pain management using a multifaceted patient-centered approach. This Patient-Centered Outcomes Research Institute (PCORI)-funded study uses multiple strategies to leverage the experience and expertise of patient and clinical stakeholders.

Methods: This pragmatic randomized trial of a behavioral intervention was conducted in two busy primary care clinics in an integrated health care system. Adult patients using prescription opioids for at least 3 months were identified using electronic health records. After consent, patients were randomized into either the intervention arm or the usual care arm. The intervention consisted of four group sessions, led by a clinical psychologist, focusing on patient activation skills, pain management, opioid education and communication strategies with providers. Baseline and follow-up telephone interviews at 6 and 12 months measure patient-centered outcomes, including quality of life, pain severity, functional status, opioid use, patient satisfaction and self-management strategies. We engaged stakeholders in study design, curriculum development, data collection, data interpretation and dissemination. Our stakeholder panel consists of 5 patients with pain ( 3 from Kaiser Permanente and 2 from a federally qualified health center), clinicians from primary care, emergency medicine, psychiatry, pain management and pharmacy, a patient advocate and an external researcher. A small number of qualitative interviews will be conducted with providers in primary care about their experience prescribing opioids.

Results: More than 2,400 potentially eligible patients were screened, and 377 patients were enrolled over 13 months. Six- and 12-month follow-up interviews are currently being conducted. Qualitative findings suggest difficulties in recruiting this complex patient population, which face considerable barriers to participation. Patients report distress over a more conservative prescribing environment, difficulties obtaining opioids, living with chronic pain and challenges communicating with providers. Qualitative findings and baseline data will be presented. Methodological considerations for conducting patientcentered research in primary care will be addressed, and the benefits of engaging stakeholders in pragmatic trials will be discussed.

Conclusion: By collaborating with a wide range of stakeholders, we hope to measure the effectiveness of a behavioral intervention on patient-centered outcomes and extend the impact of the findings beyond the health care system.

P15.04

Acculturation and Patient-Reported Experience With Health Care: An In-Depth Examination Using CG-CAHPS Surveys, Electronic Health Records, and Patient Surveys

Shuting (Lily) Liang, $^{1}$ Sukyung Chung, ${ }^{1}$ Katie Gillespie, ${ }^{1}$ Lu Wah Hung, ${ }^{1}$ Yasmin Hernandez, ${ }^{1}$ Meghan Halley ${ }^{1}$

${ }^{1}$ Palo Alto Medical Foundation Research Institute, Palo Alto, $C A$

Background: Asians in the United States, particularly South Asians and Chinese (ie, groups of more recent immigration history), report poorer health care experiences than nonHispanic whites. Few studies have examined the variation in patient-reported experience in relation to acculturation. This study aims to fill this gap using data from the CAHPS ${ }^{\circledR}$ Clinician and Group Survey (CG-CAHPS), linked electronic 
health records (EHR) and a patient acculturation survey to provide an in-depth understanding of the role of acculturation in evaluating health care experiences among South Asian and Chinese patients.

Methods: A random sample of South Asian, Chinese and non-Hispanic white patients in a multispecialty ambulatory practice in California were selected for recruitment. A total of 69 Chinese, 40 South Asians and 22 non-Hispanic whites completed the CG-CAHPS survey evaluating their most recent visit. South Asian and Chinese patients also completed a 12-item validated acculturation survey that assesses language use, media use and social relationships. We conducted multivariate logistic regression models to evaluate the effect of acculturation on patients' on CG-CAHPS reports regarding "see the provider within 15 minutes," "provider spend enough time with you," "provider explain in an way that was easy to understand" and "overall ratings of provider," taking into account the actual wait time, type of visit, history with provider recorded in the EHR of the surveyed visit, and patient's self-rated health status.

Results: South Asians and Chinese reported poorer experience than non-Hispanic whites in multiple aspects of care assessed by the CG-CAPHS survey, given similar care provided (eg, actual wait time and time with provider). Acculturation appeared to be a significant predictor of their reported experience, with varying effects between South Asians and Chinese. With regard to doctorpatient communication and overall ratings of providers, acculturation has a positive effect on Chinese patients' reported experiences but exerts no significant effects on South Asians. In terms of wait time and time spent with providers, however, acculturation has no significant effects on Chinese patients but exerts a slightly positive effect on South Asian patients.

Conclusion: Acculturation affects South Asian and Chinese to varying extents across different aspects of care. Health systems may consider targeted strategies to expand culturally competent care for different Asian populations.

\section{P15.05}

\section{Are Asians Harder to Please? A Mixed-Methods Study of Racial/Ethnic Differences in Expectations and Evaluation of Health Care Experiences}

\author{
Shuting (Lily) Liang, ${ }^{1}$ Sukyung Chung, ${ }^{1}$ Katie Gillespie, ${ }^{1}$ \\ Lu Wah Hung, ${ }^{1}$ Yasmin Hernandez, ${ }^{1}$ Meghan Halley ${ }^{1}$
}

${ }^{1}$ Palo Alto Medical Foundation Research Institute, Palo Alto, $C A$

Background: While Asians in the United States report poorer health care experiences than non-Hispanic whites, the underlying reasons for such difference remain unclear. We examined how expectations and norms surrounding evaluation among non-Hispanic whites, Chinese and South Asians, as applied to a standardized health care experience, explain the differences in reported health care experiences. Methods: A purposeful sample of 10 non-Hispanic whites, 11 Chinese and 12 South Asian patients were recruited from a large multispecialty ambulatory care practice in California. Participants first read a vignette describing an office visit and then were asked to evaluate the vignette using an experience of care (EOC) survey that consisted of 17 items selected from the CAHPS ${ }^{\circledR}$ Clinician and Group Survey (CG-CAHPS). After completing the EOC survey independently, participants took part in a cognitive interview to describe their interpretations of each EOC survey item, reasons for their rating choices and suggested improvements needed to meet their expectations. We conducted comparative analyses across racial/ethnic groups for 1) the proportion of "top-box" (the best) responses for each EOC survey item, 2) evaluation norms characterized by the "top-box" responses paired with corresponding narratives regarding "improvement needed," and 3) narratives surrounding expectations, experiences and evaluation norms for each aspect of care on the EOC survey.

Results: Non-Hispanic whites were more likely to give the "top-box" ratings and concordantly indicate "no improvement needed" compared to South Asians $(\mathrm{P}<0.05)$ and Chinese $(\mathrm{P}<0.05)$ patients. Regardless of the rating given, both Chinese and South Asians were more likely than non-Hispanic whites to avoid the "top box" and indicate "improvement needed" $(\mathrm{P}<0.05)$, echoed by their narratives reporting higher expectations regarding timeliness of visits, communicating with providers, and getting tests and referrals, which were often unmet. A common belief expressed by both Asian groups was that "top-box" ratings are reserved for situations that dramatically exceeded their expectations, which might in part explain their lower likelihood to give "top-box" ratings than non-Hispanic whites for the same situation.

Conclusion: Higher expectations among South Asian and Chinese patients may in part explain their poorer reported experiences. Further, the same care experience tends to be rated as poorer by Asians than non-Hispanic whites, which may also contribute to the lower ratings of health care experiences among Asian patients.

\section{P15.06}

Towards Culturally Competent Care: Perspectives From Both Physicians and Patients

Shuting (Lily) Liang, ${ }^{1}$ Lu Wah Hung, ${ }^{1}$ Yasmin Hernandez, ${ }^{1}$ Katie Gillespie, ${ }^{1}$ Meghan Halley, ${ }^{1}$ Sukyung Chung ${ }^{1}$

${ }^{1}$ Palo Alto Medical Foundation Research Institute, Palo Alto, $C A$

Background: Patient satisfaction has become an increasingly important health care quality indicator and been widely used to compare quality across health care organizations and 
providers. At a large multispecialty ambulatory care practice in California, a notable difference across regions has been recognized, with sites serving higher proportions of Asian patients tending to receive significantly lower satisfaction scores. The current study aims to identify the key drivers of this issue and provide recommendations for strategies to improve culturally competent care across health systems.

Methods: We conducted 18 focus groups and 32 interviews with a total of 69 Chinese and 40 South Asian patients as well as 7 in-depth interviews with primary care physicians serving a significant proportion of Chinese and South Asian patients. All data were transcribed verbatim and coded with Dedoose, a multifunctional qualitative analysis software. First, data were coded by aspects of care. Then, an inductive thematic approach was used to identify themes around language barriers and racial/ethnic/cultural differences in relation to care. Further, patient-reported themes were triangulated with physicians' accounts to draw recommendations for expanding culturally competent care.

Results: Both patients and providers emphasized that an understanding of Asian patients' beliefs about health and illness, which are fundamentally different from Western medicine, is an imperative to deliver culturally competent care. Knowledge of the social norms in interpersonal and family interactions also was considered important. For Chinese and South Asian patients in particular, more education is needed on the use of lab tests, imaging and antibiotics. Moreover, patients with lower English proficiency in both groups reported language as a barrier to satisfactory experience. Translation services were not favored by either patients or physicians because they are perceived to be time-consuming and of low quality. While patients reported frustration in scheduling with physicians who understand both their language and culture, such physicians reported being overwhelmed with workload. Physicians also voiced the need for more medical assistants and receptionists with the language capacity to support their care.

Conclusion: Culturally competent care requires knowledge of cultural beliefs and practices in relation to health as well as language support from the care team and the health system.

\section{P15.07}

\section{Response to Survey Solicitation to Patient Portal Members Differs by Age, Race, and Health Care Utilization}

Cathryn Peltz-Rauchman, ${ }^{1}$ George Divine,${ }^{1}$ Daniel McLaren, ${ }^{1}$ Ko Un Park, ${ }^{1}$ Ilan Rubinfeld, ${ }^{1}$ Michelle Schreiber, ${ }^{1}$ David Allard, ${ }^{1}$ Christine Cole Johnson ${ }^{1}$

${ }^{1}$ Henry Ford Health System, Detroit, MI

Background: Health care systems are increasingly utilizing electronic medical record-associated patient portals to facilitate communication with patients and between providers and their patients. These patient portals are growing in recognition as potentially valuable research tools. However, while there is much known about the demographics of patients who are portal members (older, white), not much is known about which portal members respond to surveys solicited within that specific population. Therefore, the objective of this study was to determine the demographics of patient portal users who respond to a survey request.

Methods: A one-time email with a link to a survey through REDCap was sent to 10,015 randomly selected MyChart users. This survey included questions regarding the timing of the release of both routine and potentially sensitive (eg, biopsy, genetic or sexually transmitted disease) test results. Two weeks were allowed for survey completion.

Results: The survey had a $13 \%$ response rate $(n=1,303)$, which varied by several demographic characteristics. Specifically, the adjusted odds ratios indicated that, on average, a 10-year increase in age corresponds with higher odds of responding to the survey (odds ratio [OR]: 1.40; $\mathrm{P}<0.001)$. Race was a significant factor, as users selfidentified as black (OR: 0.50; $\mathrm{P}<0.001)$ and other $(\mathrm{OR}$ : $0.74, \mathrm{P}<0.001)$ races were less likely to respond than those self-identified as white. Patients who averaged more than one visit to a specialist per year over the last 2 years were more likely to respond than those who averaged one or fewer visits (OR: 1.32, $\mathrm{P}<0.001$ ), with similar results for primary care provider visits (OR: $1.22 ; \mathrm{P}=0.02$ ).

Conclusion: We found that there are demographic differences in respondents to a survey solicited to a random sample of active patient portal users. Respondents tended to be older, white and more frequent users of care from both specialist and primary care physicians. Patient portals are potentially valuable tools for research; however, it is important to understand that respondents to surveys solicited to this sampling frame may not be entirely representative and that additional approaches to engage a wide range of participants are likely necessary.

\section{P15.08 \\ Preference for Immediate Release of Test Results Through a Patient Portal Differs by Demographics and Type of Results}

Cathryn Peltz-Rauchman, ${ }^{1}$ George Divine ${ }^{1}$ Daniel McLaren, ${ }^{1}$ Ko Un Park, ${ }^{1}$ Ilan Rubinfeld, ${ }^{1}$ Michelle Schreiber, ${ }^{1}$ Christine Cole Johnson, ${ }^{1}$ David Allard ${ }^{1}$

\section{${ }^{1}$ Henry Ford Health System, Detroit, MI}

Background: Electronic medical record (EMR) use has increased dramatically over the past 10 years on a national scale, and associated patient portals provide a direct and secure environment in which patients can receive information from their health care providers, leading to unprecedented advances in communication 
between providers and patients. While previous studies have shown patients are in favor of receiving test results through such portals, preferences specific to the type of result, and if these preferences change with certain patient characteristics, are unknown. The objective of this study was to determine differences in patient preferences for the release of test results through an EMR-associated portal.

Methods: A one-time email with a link to a survey through REDCap was sent to 10,015 randomly selected patient portal users. The survey had questions regarding the release of "regular" test results and the release of "sensitive" results (including genetic testing, sexually transmitted disease and biopsy results). Two weeks were allowed for survey completion. Variables assessed included age, race, sex, marital status, comorbidities, specialty and primary care physician visits, and insurance type. Analysis was performed in $\mathrm{R}$ software and statistical significance set at $\mathrm{P}<0.05$.

Results: The survey had a $13 \%$ response rate $(n=1,303)$. Most patients preferred their "regular" results released to them immediately (86\%) while the remaining patients preferred a built-in automatic delay. Preferences regarding the release of "regular" results did not differ by patient characteristics. Only $59 \%$ of patients preferred "sensitive" results released immediately, with female patients more likely to prefer a delay in the release of sensitive results (odds ratio: 1.45; $\mathrm{P}=0.01$ ). Preference regarding release of "sensitive" results did not differ by other patient characteristics.

Conclusion: We found patient preference to vary with the type of result (regular or sensitive), but the only difference in preference specific to patient characteristic was that of female patients preferring a delay in the release of sensitive results. With the growing availability of patient-provider communication through EMRs and the importance of incorporating patient-centered approaches into health care, it may be advantageous to tailor the release of results to patient preferences, such as adjusting release by the type of result.

\section{QUALITATIVE METHODS AND APPLICATIONS}

P16.01

Develop Patient-Focused Educational Materials and Scripting for a Randomized Trial to Reduce Opioid Use Following Total Hip and Total Knee Arthroplasty

David Smith, ${ }^{1}$ Lynn DeBar, ${ }^{1}$ Jennifer Kuntz, ${ }^{1}$ Jill Mesa, ${ }^{1}$ Jennifer Schneider ${ }^{1}$

${ }^{1}$ Kaiser Permanente Center for Health Research, Portland, OR

Background: The American Academy of Orthopaedic Surgeons recognizes the unintended consequences of focusing on opioids for pain management.
Methods: This was a qualitative study with open-ended, structured interviews $(n=18)$ at Kaiser Permanente Northwest. A purposeful sampling method identified surgeons, advice nurses, physical therapists, physician assistants and patients. Patients were recent total hip/ knee arthroplasty (THA/TKA) cases in the top third of opioid use after surgery. Interview guides captured feedback consistently. Provider interviews explored their approach with THA/TKA patients on: pain management, barriers to opioid titration, and recommendations/changes on educational materials to support pain management and opioid reduction. Patient interviews explored their experience, understanding and beliefs surrounding opioids, and recommendations on important content. A qualitative methodologist conducted interviews and content analysis to identify key themes.

Results: Recommendations for content in patient educational materials and scripting included: 1) Clear descriptions of how opioids work in the body, how to taper, nonopioid pain management options, and problems from overuse (eg, side effects and pain masking); 2) Messaging on how long to expect to use opioids and type of pain to expect; 3) Providing a visual timeline for patients to reinforce pain medication titration expectations and home or physical therapy exercises, especially during the acute phase; 4) Emphasis on the multimodal approach to pain management and the importance of a balance between opioids for recovery versus overuse; 5) Explanations to chronic opioid users that their pain and its management may vary from opioid-naïve patients; and 6) Provide education and messaging multiple times prior to and after surgery.

Conclusion: Patients and providers agreed that clearly stated verbal and written messaging is needed beyond what has typically been done regarding opioid expectations; the resulting materials are being tested in an ongoing trial.

\section{RESEARCH METHODS, ANALYTIC TOOLS AND DESIGNS}

\section{P17.01 \\ Technologies for Managing Virtual Data Warehouse Access and Identifying Appropriate Levels of Staffing at $\mathrm{CHI}$ Institute for Research and Innovation}

David Bailey ${ }_{1}{ }^{1}$ John Weeks, ${ }^{1}$ Elizabeth Evans, ${ }^{1}$ Jan Lowery, ${ }^{1}$ Lela McFarland ${ }^{1}$

${ }^{1}$ Catholic Health Initiatives, Englewood, CO

Background: CHI Institute for Research and Innovation (CIRI) has implemented a number of tools to enable users to achieve self-service access to its virtual data warehouse (VDW) and make requests for additional data (not provided 
by VDW self-service). In addition to our tools for managing the access to historic retrospective data, we have integrated a clinical trial management system for protocol management, study accounting, electronic data capture and patient management. Identifying tools that can help automate delivery and monitor demand are essential components of our technology strategy.

Methods: Our strategy for developing or selecting technology assets has been guided by the following factors: 1) functionality - does the tool do what we need it to do or desire it to do? 2) usability - is the tool or technology easy to learn and use for persons of all levels of ability, and can it be used broadly to support various types of research? 3) security - does the technology provide adequate measures to ensure security of data stored and/or accessed? 4) interoperability with other tools; and 5) cost of the technology, initial and long term, with consideration of open source options.

Results: To date, we have implemented tools for clinical trial management of both sponsor- and investigatorinitiated trials, a data query tool to allow end-users to access the VDW to perform simple queries and prep-toresearch activities, electronic data capture for collecting study-specific data and patient-reported outcomes, and a grant management system. We also have implemented a tool for tracking project deliverables and staff time, which we are using to quantify demand and identify need for additional resources. We are currently evaluating tools for data visualization and for streamlining acquisition of additional data assets to compliment the VDW.

Conclusion: Having a strategy around development and implementation of technology assets is critical for ensuring safe and broad access to our data resources, and for monitoring demand among users in order to identify needs for appropriate resources. These tools will enable CIRI to expand the scope of research conducted at Catholic Health Initiatives and to create a network of researchers who can collaborate on studies without being co-located by creating virtual research organizations across our facilities.

\section{P17.02}

\section{Feasibility of Collecting Patient-Reported Outcomes After Major Cancer Surgery: A Survey of Kaiser Permanente Members Six Months After Cystectomy for Bladder Cancer}

\author{
Joanna Bulkley, ${ }^{1}$ Maureen O'Keeffe Rosetti, ${ }^{1}$ \\ Christopher Wendel, ${ }^{2}$ James Davis, ${ }^{1}$ Michael Leo, ${ }^{1}$ \\ Sheila Weinmann, ${ }^{1}$ Julie Munneke, ${ }^{3}$ Teresa Harrison, ${ }^{4}$ \\ Marilyn Kwan, ${ }^{3}$ Kim Danforth, ${ }^{4}$ Carmit McMullen ${ }^{1}$ \\ ${ }^{1}$ Kaiser Permanente Center for Health Research, Portland, \\ OR; ${ }^{2}$ University of Arizona College of Medicine, Tucson, AZ; \\ ${ }^{3}$ Kaiser Permanente Division of Research, Oakland, CA; \\ ${ }^{4}$ Department of Research \& Evaluation, Kaiser Permanente \\ Southern California, Pasadena, CA
}

Background: Researchers seeking to understand the physical, socioemotional and quality-of-life impacts of serious health conditions such as cancer need to interact with patients during times of poor health if they hope to develop a more complete understanding of the patient experience. A common concern is these patients will be overburdened if asked to participate in research and that participation rates will be low. Our analysis describes recruitment methods and examines survey response rates among bladder cancer patients in the period after bladder removal surgery, when they may be facing health challenges and are adjusting to altered urinary function.

Methods: We conducted focus groups with bladder cancer patients with urinary diversions to identify topic domains and develop a patient-reported outcomes questionnaire. We then conducted a survey 6 months postsurgery at three Kaiser Permanente sites. Eligible patients had bladder removal and reconstruction surgery as identified from the electronic health record and confirmed through chart review. Patients were mailed a cover letter and a 30-page questionnaire, which included fixed-choice and open-ended questions on quality of life, health care decision-making, urinary function, sexual function, body image, coping and health care expenses. We placed up to 10 phone calls to nonrespondents. As explained in the cover letter, return of the survey was considered consent to participate.

Results: The participation rate was $69 \%$ (site range: $63 \%-$ $78 \%$ ); $88 \%$ of participants completed $95 \%$ or more of the scale items. The scales with the most missing items proportionally were spiritual well-being (8.9\%), urinary function (5.9\%), goal dissonance $(5.6 \%)$ and decision regret (4.5\%). Among 269 participants, $76 \%$ provided an estimate of total out-of-pocket expenses since bladder cancer surgery and $62 \%$ provided at least one estimate for specific categories of expenses.

Conclusion: Our results suggest that even during periods of serious health challenges, patients will participate in research studies and are willing to complete long questionnaires when they are highly relevant to their condition. Focus group reports from our study indicate that patients desire the opportunity to be heard and to share their experience, and that in doing so they hope to contribute to improving care for future bladder cancer patients.

P17.03

Enrolling Patients in the Sutter Health Biobank:
Lessons Learned From Testing Different
Recruitment Methods

Katherine Gillespie, ${ }^{1}$ Yasmin Hernandez, ${ }^{1}$ Lu Wah Hung, ${ }^{1}$ Harold Luft ${ }^{1}$

${ }^{1}$ Palo Alto Medical Foundation Research Institute, Palo Alto, $C A$

Background: Multiple methods may be used to recruit patients for research. These vary in resources required, 
including monetary costs and staff time, as well as in "yield," the proportion of people approached who agree to participate. Biobanking is a new and growing area of health research that presents a variety of logistical considerations from recruitment to enrollment.

Methods: A pilot study was conducted to test different strategies for the Sutter Health Biobank recruitment of a general/healthy population sample. Patients were first contacted either by an email or a traditional letter. Patients could choose to not be further contacted about the study. Follow-up contact options included an email, letter or phone call. The initial recruitment material invited the patient to a website with more information about the biobank and a consent form. After a patient accesses the site and either accepts or declines participation, a brief quality improvement question was asked about why they made their decision.

Results: The results of the pilot study will describe the outcomes of each recruitment strategy from the staff time and cost per number of enrolled biobank participants. Demographic factors about those who participate, decline participation or do not respond will be available from the electronic health records for additional context.

Conclusion: Different recruitment methods have different tradeoffs in terms of effort put in and the outcome of biobank enrollment. These results may indicate that certain strategies are more effective for some populations compared to other populations. For hard-to-reach patients or for those with more questions or concerns, a phone call may encourage reviewing the biobank materials before deciding whether to accept or decline participation.

\section{P17.04}

\section{Identification of Migraine at Sutter Health: An Application of an EHR-Based Algorithm}

Alice Jacobson, ${ }^{1}$ Heather Law, ${ }^{1}$ Andrew Avins, ${ }^{2}$ Sylvia Sudat, ${ }^{1}$ Jessica Liu, ${ }^{1}$ Lisa Dean, ${ }^{1}$ Alice Pressman ${ }^{1}$

${ }^{1}$ Sutter Health, Walnut Creek, CA; ${ }^{2}$ Kaiser Permanente Division of Research, Oakland, CA

Background: Migraine is one of the most common and disabling disorders in the world. In the United States, 1 in 4 households is affected by it. With no diagnostic test, migraine is most commonly detected through patient interview and rule out of other causes of headache. Studies to identify patient populations for prevalence estimates or clinical research often involve costly and time-consuming population-based surveys or data collected from specialty headache clinics. To identify migraineurs in Sutter Health, we extracted data from electronic health records (EHR) and applied a migraine probability algorithm (MPA) that we previously developed in a separate large integrated health care system. Our goal was to identify a target population of migraineurs from which to recruit for future studies.

Methods: We identified all migraine diagnoses, migraine- specific prescriptions and migraine listed as a significant health problem from the EHR for the 5-year period November 2010 through October 2015. Applying the MPA, we calculated a migraine probability for all patients in the Sutter Health primary care population, defined as adults with one or more visits to primary care during the study period. We report prevalence estimates by age, sex and race.

Results: The prevalence of medically ascertained migraine among the Sutter Health primary care population was $7.3 \%$ overall. Migraine was more prevalent among women than men, $9.8 \%$ and $3.4 \%$ respectively, and peaked for women aged 35-54 years (12.2\%). Migraine prevalence was highest among whites (8.4\%) and lowest among Asians (4.4\%).

Conclusion: Our study confirms that using EHR data along with the MPA is an inexpensive and easily applied method of identifying migraineurs for use in research. We have calculated prevalence estimates at Sutter Health and will use this methodology to identify potential participants for a concurrent National Institutes of Health trial of mindfulness for migraine. Although our calculated prevalence estimates are lower than those reported in the literature, we believe this is due to the restriction that patients utilize Sutter Health primary care services. To test this, we included specialty care patients and identified as many as 3\% more migraineurs, thus warranting further study. Future plans include a patient survey to validate the MPA in the Sutter Health patient population.

\section{P17.05}

Fidelity Measurement of a Mindfulness-Based Stress Reduction Intervention in a Randomized Controlled Feasibility Trial for Migraine Patients

Heather Law, ${ }^{1}$ Bob Stahl, ${ }^{2}$ Andrew Avins, ${ }^{3}$ Sylvia Sudat, ${ }^{1}$ Alice Jacobson, ${ }^{1}$ Jessica Liu, ${ }^{1}$ Marina Dolginsky, ${ }^{1}$ Lisa Dean, ${ }^{1}$ Alice Pressman ${ }^{1}$

${ }^{1}$ Sutter Health, Walnut Creek, CA; ${ }^{2}$ Awareness \& Relaxation Training, Santa Cruz, CA; ${ }^{3}$ Kaiser Permanente Division of Research, Oakland, CA

Background: Mindfulness-based stress reduction (MBSR) is an 8-week meditation-based intervention developed by Jon Kabat-Zinn at the University of Massachusetts Medical Center (UMass) in the 1970s. Increasing evidence has shown effectiveness of MBSR for many patients with a variety of pain-related and functional disorders. In preparation for a fully powered randomized controlled clinical trial of MBSR for patients with moderate-to-severe migraine headache, we will conduct a two-arm, parallel-comparison randomized controlled feasibility trial of MBSR versus usual care for 60 migraine patients at a large health system. Documenting fidelity for practitioner-delivered interventions is a critical aspect of these trials for ensuring that delivery of the intervention adheres to consistent standards for integrative 
health care. Fidelity measurement is one of the main outcomes for this pilot study.

Methods: MBSR instructors will use a standardized curriculum developed at UMass. MBSR content oversight will be provided by a senior teacher who has founded several MBSR programs in health care settings. He will have primary responsibility for ensuring fidelity of the MBSR intervention by meeting with each teacher on a monthly basis to ensure adherence to the standard curriculum. He and the research team also will create an MBSR "fidelity checklist" for use by a research specialist who will record and listen to a randomly selected session for each instructor in order to monitor the teaching.

Results: Fidelity measurement is defined as the extent to which delivery of an intervention adheres to the protocol or program model as originally developed. Despite its importance, there has been little development of tools for measuring fidelity and evaluated methods for documenting the degree of fidelity in a clinical trial. This presentation will describe the details of our fidelity-assessment methodology; the MBSR "fidelity checklist" developed for this trial also will be shared for adaptation by other investigators conducting similar studies.

Conclusion: Proven fidelity of the MBSR intervention is needed for the results of this study to be generalizable for a fully powered phase III trial. In addition, the information we gain from fidelity measurement will help inform future studies of MBSR for migraine and other conditions.

\section{P17.06}

\section{Stakeholders' Views on Data Sharing and Multisite Research}

Kathleen Mazor, ${ }^{1}$ Marsha Raebel, ${ }^{2}$ David Arterburn, ${ }^{3}$ Allison Richards, ${ }^{1}$ Mia Gallagher, ${ }^{4}$ W. Benjamin Nowell, ${ }^{5}$ Darren Toh ${ }^{4}$

${ }^{1}$ Meyers Primary Care Institute, Worchester, MA; ${ }^{2}$ Kaiser Permanente Colorado, Denver, CO; ${ }^{3}$ Group Health Research Institute, Seattle, WA; ${ }^{4}$ Harvard Pilgrim Health Care Institute, Boston, MA; ${ }^{5}$ Global Health Living Foundation, Nyack, NY

Background: Data sharing is a fundamental step in multisite studies, enabling research on rare outcomes, treatment heterogeneity and greater generalizability. However, data sharing entails costs and risks. Newly developed privacypreserving analytic and data-sharing methods offer an approach to sharing data and conducting multisite research that eliminates the need to share identifiable patient-level information. Identifying stakeholders' perceptions and synthesizing overarching themes about willingness to share data are important first steps toward increasing the acceptability and use of these new tools. We therefore sought to understand stakeholders' views on data sharing generally and privacy-preserving methods in particular.
Methods: We conducted semi-structured group and individual interviews with a purposive sample of stakeholders to gather a variety of perspectives on data sharing in general and the use of privacy-preserving methods (PPM) specifically. Interviews were audio-recorded and professionally transcribed. Using content coding followed by thematic coding, we sought to identify factors affecting stakeholders' willingness to share data, with particular attention to the potential impact of privacypreserving methods.

Results: A total of 11 stakeholder interviews were completed, involving patients $(n=15)$, researchers $(n=10)$, institutional review board and regulatory staff $(n=3)$, multicenter research governance $(n=2)$ and health care system leaders $(n=4)$. Stakeholders' perceptions of the benefit and value of the research was the strongest influence toward data sharing; perceived value was related to the relevance of the scientific question and the methodologic rigor. Influences against data sharing were primarily cost and data-security risks; the latter was mitigated by various safeguards (encryption, data use agreements, oversight), successful data sharing experience, established relationships and trust. The risk reduction obtained by sharing aggregate data rather than individual-level data was acknowledged as being potentially more acceptable to some stakeholders, but interviewees also expressed concerns about the increased cost and questioned whether aggregating data resulted in a loss of information that would in turn lessen the value or validity of the research.

Conclusion: The gains in privacy protection associated with the use of privacy-preserving methods in multisite studies involving data sharing were attractive to some stakeholders, but factors such as the value and generalizability of the research appear potentially more influential.

\section{P17.07}

\section{Direct Adjustment of Obesity Estimates in the Colorado BMI Monitoring System}

\section{Liza Reifler, ${ }^{1}$ Eric Bell, ${ }^{2}$ David Tabano, ${ }^{1}$ Jennifer Barrow, ${ }^{1}$} LeeAnn Rohm, ${ }^{1}$ Rickey Tolliver $^{2}$ Matthew Daley ${ }^{1}$

${ }^{1}$ Kaiser Permanente Colorado, Denver, CO; ${ }^{2}$ Colorado Department of Public Health and Environment, Denver, CO

Background: In the Colorado BMI Monitoring System, electronic health record body mass index (BMI) data from participating health care organizations are provided to the Colorado Department of Public Health and Environment (CDPHE) and combined to establish BMI estimates at the census tract level. This system provides estimates with more geographic specificity than national surveys; however, population representativeness is a limitation. Data are sampled from all members of five health care organizations, and selection bias is possible.

Methods: We applied direct adjustment based on gender, race/ethnicity and age to estimate BMI and overweight/ obesity prevalence estimates. To avoid limiting to complete 
case data, missing race/ethnicity data were imputed using hot decking based on census tract, gender and age classes. Raking, an iterative method of marginal weight adjustment, was used to create sample weights used in direct adjustment estimates by census tract. Hot decking and raking were performed using modified SAS macros developed by Abt Associates (Cambridge, MA).

Results: Processes were developed on one site's 2012-2014 data $(\mathrm{N}=479,960)$ and are being tested on all site data at CDPHE. Missing race was imputed for $13.2 \%$ of members and failed on only $0.1 \%$. Individual weights were generated through raking for $99.5 \%$ of individuals with a recent BMI measurement. For 658 of 668 Denver-metro census tracts, crude and adjusted estimates were similar (Pearson $\mathrm{R}=0.981, \mathrm{P}<0.01$ ), and the median absolute difference in crude versus adjusted adult obesity prevalence estimates was 0.05 (interquartile range: $-0.54-0.80$ ).

Conclusion: It is feasible to apply direct standardization to large data systems with many geographic units. Imputation via hot decking is appealing because it has been used in large government and public health surveys, it is effective using a limited set of demographic variables, and it provides a reasonable estimate of variable distribution by drawing from observed values. Raking is an advantageous weighting method in direct adjustment because it avoids empty or small cell size and only requires populationmarginal demographic group estimates. Overall, adjusting census tract obesity prevalence estimates modified values slightly (the majority of absolute difference of crude and adjusted was within $1 \%$ in either direction), and adjusted estimates created more conservative confidence limits.

\section{$\mathrm{P} 17.08$}

\section{Sleep Deprivation in Hospitalized Patients Over the Age of 60: An Approach to Recruitment Challenges in an Inpatient Setting}

\author{
Ariana Ruiz, ${ }^{1}$ Alice Pressman, ${ }^{1}$ Katie Stone, ${ }^{1}$ Lisa \\ Dean, ${ }^{1}$ Sarah Robinson, ${ }^{1}$ Phillip $Y u^{1}$
}

${ }^{1}$ Sutter Health, Walnut Creek, CA

Background: Chronic sleep deprivation in older adults contributes to dementia, cardiovascular disease and mortality, but has not been studied in a hospital setting. We conducted a pilot study in a Northern California hospital to evaluate the quantity and quality of sleep among inpatients age 60 years and older. The study goal was to evaluate the association between sleep and hospital outcomes such as length of stay (LOS), delirium and readmissions. The focus of this abstract is to describe our approach to recruitment challenges in a hospital setting.

Methods: This study was conducted at an 81-bed community acute-care hospital in rural Tracy, California. Our goal was to recruit 100 patients in a 1-year period. We began with strict eligibility criteria, exclusively recruiting patients admitted with an expected LOS $\geq 2$ days and without cognitive impairments. We measured sleep with 24-hour wrist actigraphy and sleep diaries, and collected patient-reported outcomes through in-person questionnaire. Study staff included more than 25 volunteers from local universities. We also included an education component for bedside nursing staff and volunteers.

Results: In the first 5 months we recruited 12 patients and worked with leadership to revise eligibility requirements and recruitment methods in an attempt to increase yield. Specifically, we relaxed the 2-day expected LOS and extended recruitment efforts to the emergency department. We also added Spanish-language materials. In the 4 months since making these changes, we have recruited 28 patients. We determined that the Spanish materials and recruitment effort from the emergency department did not impact recruitment rates; however, modifications to the eligibility criteria led to better recruitment.

Conclusion: There are several important elements to successful recruitment and participant retention for research in a hospital setting. Flexibility and creativity are essential; in our case, we relaxed restrictions on estimated LOS. In addition, having assistance from highly trained volunteers and nursing staff was equally valuable. Finally, the support of leadership (chief medical executive and nursing administration) allowed us to make some of the necessary changes throughout the process. With our new processes in place, we hope to complete recruitment within 6 months.

\section{P17.09}

\section{Identification of Incident Uterine Fibroids Using Electronic Medical Record Data}

\section{Onchee Yu, ${ }^{1}$ Renate Schulze-Rath, ${ }^{2}$ Susan Reed, ${ }^{3}$ Jane Grafton, ${ }^{1}$ Kelly Hansen, ${ }^{1}$ Delia Scholes ${ }^{1}$ \\ ${ }^{1}$ Group Health Research Institute, Seattle, WA; ${ }^{2}$ Bayer Pharma $A G$, Berlin, Germany; ${ }^{3}$ University of Washington, Seattle, WA}

Background: Uterine fibroids are the most common benign tumors of the uterus that are associated with considerable morbidity in women. Diagnosis codes have been used to identify symptomatic fibroid cases, but their accuracy, especially for incident cases, is uncertain. This study assessed the accuracy of diagnosis codes in identifying incident fibroids and developed algorithms to improve incident fibroid case-finding using additional electronic data.

Methods: Women aged 18-65 years who received an ICD9 diagnosis code for uterine fibroid during 2012-2014 were identified from electronic databases at Group Health Cooperative, an integrated health care system in Washington State. Women with a fibroid history or hysterectomy were excluded. Medical records were reviewed on a random sample of 617 women to confirm incident fibroid status. Additional data on demographics, symptoms, treatment, imaging, health care utilization, comorbidities and medication 
were collected. Classification and regression tree analysis incorporating these additional data were used to develop algorithms to identify incident fibroid. We focused on an algorithm with high sensitivity (ie, maximizing the inclusion of true incident cases) and another with high specificity (ie, avoiding incorrect inclusion of noncases as incident cases). Algorithm performance was assessed by calculating sensitivity, specificity and positive predictive value (PPV) using medical record as gold standard.

Results: Among the 617 women, mean age at diagnosis was 48 years. Medical record review confirmed 583 (95\%) fibroid cases and 482 incident cases, a 78\% PPV for incident cases based on diagnosis codes alone. Incorporating additional electronic data, the algorithm classified 395 incident cases among women with at least 2 pelvic ultrasounds on and prior to diagnosis date. Of these, 344 were correctly classified as incident cases, yielding an $87 \%$ PPV. Sensitivity was $71 \%$ and specificity $62 \%$. A second algorithm further classified women based on a fibroid code of 218.9 in 2 years after diagnosis and lower body mass index yielded 93\% PPV, $53 \%$ sensitivity and $85 \%$ specificity.

Conclusion: Identification of incident uterine fibroids through ICD-9 diagnosis codes alone was good with moderate PPV. Algorithms using additional electronic data improved incident fibroid case finding with higher PPV, and either higher sensitivity or higher specificity to meet different study aims.

(C) 2017 Aurora Health Care, Inc. 